UNIVERSTTÉ DU QUÉBEC

\author{
MÉMOIRE \\ PRÉSENTÉ À \\ L'UNIVERSITÉ DU QUÉBEC À CHICOUTIMI \\ COMME EXIGENCE PARTIELLE \\ DE LA MAÎTRISE EN ÉDUCATION (M.A.)
}

PAR

NATHALIE SIMARD

BACHELIĖRE EN ÉDUCATTON (B.Ed.)

\title{
Proposition de grille et de principes formatifs pour la correction des productions écrites
}

Mai 1996 


\section{Bibliothèque}

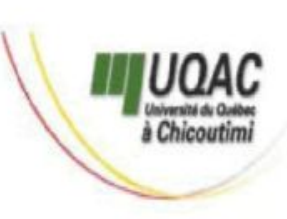

\section{Mise en garde/Advice}

Afin de rendre accessible au plus Motivated by a desire to make the grand nombre le résultat des results of its graduate students' travaux de recherche menés par ses research accessible to all, and in étudiants gradués et dans l'esprit des accordance with the rules règles qui régissent le dépôt et la governing the acceptation and diffusion des mémoires et thèses diffusion of dissertations and produits dans cette Institution, theses in this Institution, the I'Université du Québec à Université du Québec à Chicoutimi (UQAC) est fière de Chicoutimi (UQAC) is proud to rendre accessible une version make a complete version of this complète et gratuite de cette œuvre. work available at no cost to the reader.

L'auteur conserve néanmoins la The author retains ownership of the propriété du droit d'auteur qui copyright of this dissertation or protège ce mémoire ou cette thèse. thesis. Neither the dissertation or Ni le mémoire ou la thèse ni des thesis, nor substantial extracts from extraits substantiels de ceux-ci ne it, may be printed or otherwise peuvent être imprimés ou autrement reproduced without the author's reproduits sans son autorisation. permission. 


\section{REMERCIEMENTS}

Cette étude a permis une initiation à la recherche et de nombreuses expériences dans le domaine de l'éducation. L'auteure veut remercier tout spécialement son directeur de mémoire, Monsieur Raymond Claude Roy, Ph.D., professeur au Département des sciences de l'éducation de l'Université du Québec à Chicoutimi, qui s'est révélé dès le départ un excellent conseiller. Sa compétence, sa disponibilité, son dynamisme et la qualité de ses exigences ont grandement été appréciés.

L'auteure veut également remercier son co-directeur, Monsieur Joseph Morose, Ph.D., professeur au Département des sciences de l'éducation de l'Université du Québec à Chicoutimi, pour ses précieux conseils. Son expérience et sa perspicacité ont contribué à l'enrichissement de la recherche.

L'auteure désire aussi remercier les organismes subventionnaires qui lui ont accordé des bourses d'études: la Fondation Asselin et les Éditions Gaëtan Morin.

De plus, l'auteure tient à transmettre sa reconnaissance à tous les correcteurs qui ont participé aux expériences de correction entre 1992 et 1995. Il est fait allusion plus particulièrement aux chargées de cours de l'époque, Mesdames Chantale Bussières, Lise Harvey et Louise Lefebvre. Leurs judicieux conseils et leur clairvoyance ont permis de faire avancer les travaux de recherche. 
L'auteure aimerait également remercier Madame France Gauthier, M.A., pour son implication, ses remarques constructives et son intérêt face à la recherche.

Des remerciements d'ordre technique vont aussi à Madame Johanne Beaumont pour son aide précieuse lors de la mise en page du mémoire et à Monsieur Simon Forgues, informaticien, pour les conseils donnés lors de l'utilisation du logiciel File Force.

L'auteure tient enfin à remercier tous ses parents et amis qui l'ont soutenue au cours de son cheminement personnel à la maîtrise. Leur présence et leur écoute ont été d'un grand support. 


\section{RÉSUMÉ}

Cette recherche-développement propose essentiellement une grille et des principes formatifs pour la correction des productions écrites en français. Elle s'est donné trois objectifs spécifiques:

1 - Choisir et justifier les éléments d'une grille de correction des productions écrites à haute valeur diagnostique et qui mène le scripteur à rếmser des apprentissages.

2. Réconcilier la grille avec une approche nouvelle de correction formative et, par le fait même, déterminer quelques principes sous-jacents à l'utilisation d'une telle approche.

3. Élaborer, à titre de modèles, quelques exercices de soutien à la correction.

La recherche prend comme point de départ l'outil de correction utilisé dans la recherche de Boyer, Roy et Roy (1991), qu'elle modifie pour en faire une grille complète et renouvelée comptant en tout neuf grandes catégories. Les sous-catégories de la grille ont été réévaluées, modifiées ou inventées même, réorganisées pour certaines à l'intérieur de la catégorie qui les regroupe. Les changements et modifications résultent d'analyses reposant sur des vues de grammaire raisonnée, de linguistique structurale ou de simple pragmatique pour les catégories relatives à la langue, d'analyses raisonnées plus pratiques relatives à la stylistique, à la qualité textuelle et à la qualité de la pensée. Les propos de présentation permettront au maître intéressé par un outil sophistiqué de correction de saisir la nature fine des erreurs qui peuvent émailler les productions écrites et de s'initier à un matériel qui permet de transmettre au scripteur une identification diagnostique de chacune de ses erreurs, de nature à mener, par une réaction naturelle, à des apprentissages.

En complément, sur la base des données de l'évaluation formative (Scallon, 1988), puis des rapprochements faits entre ces données et les théories cognitives (Gagné, 1976; Roy, 1995), il a été possible de faire ressortir des principes de correction formative. Ces principes sont issus d'une interprétation pragmatique et ont pour but de guider l'utilisation même de la grille.

Recherche-développement, l'étude propose au maître un Guide de correction référentielle et interactive présentant pour chacune des sous-catégories une description et plusieurs exemples en illustration. Il est également mis à la disposition du maître des modèles d'exercices à mettre à la disposition des apprenants qui ont à se donner la maîtrise de certaines notions spécifiques. La recherche laisse donc au maître qui désire s'engager dans la voie d'une correction formative tout un matériel nécessaire autant à son initiation qu'à son travail pratique de correction. 


\section{TABLE DES MATIÈRES}

REMERCIEMENTS ……........................................................................................................ ii

RÉSUMÉ

TABLE DES MATİ̀RES ……......................................................................................... v

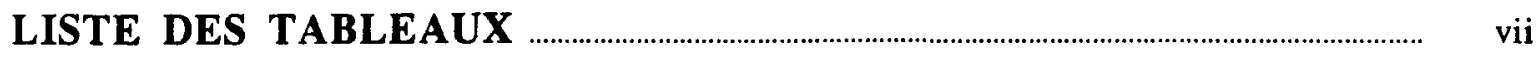

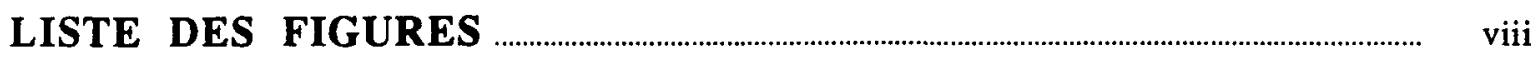

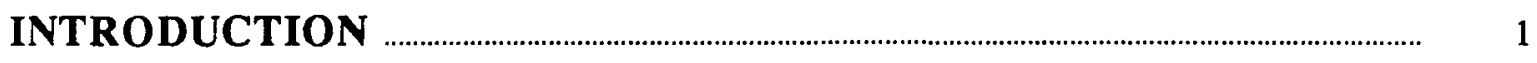

CHAPITRE PREMIER: Les travaux de recherche menés sur le thème de la maîtrise de l'écriture

1.1 Les tendances marquantes des études menées dans les vingt dernières

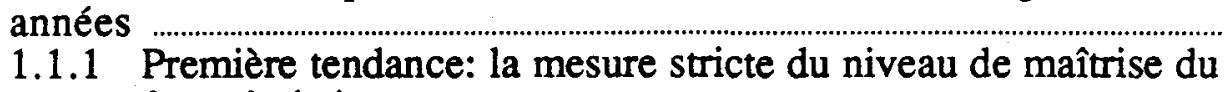
français écrit ...........................................................................................

1.1.2 Deuxième tendance: les recherches sur l'intervention en maîtrise du français écrit........................................................................................... 16

1.1.3 Troisième tendance: les recherches sur l'intervention en approche formative .............................................................................................................. 20

1.2 L'orientation et le cadre de la recherche ……............................................................ 25

1.2.1 Les objectifs de la recherche ………................................................................ 26

1.2.2 Le cadre de l'étude .................................................................................................. 28

CHAPITRE II: Les fondements et les catégories d'une grille de correction

2.1 Les fondements de l'analyse 
2.2 La grille de correction .................................................................................................... 34

2.2.1 La catégorie 100: Orthographe d'usage ................................................. 35

2.2.2 La catégorie 200: Morphologie ……..................................................... $\quad 40$

2.2.3 La catégorie 300: Accord ............................................................................... 47

2.2.4 La catégorie 400: Structure de la phrase ………....................................... 50

2.2.5 La catégorie 500: Signes de ponctuation et conventions .................. 55

2.2.6 La catégorie 600: Lexique ……............................................................ 59

2.2.7 Les catégories 700, 800 et 900: Stylistique, Qualité textuelle et Qualité de la pensée

CHAPITRE III: L'évaluation formative des productions écrites ……........ 72

3.1 L'évaluation formative …….....................................................................................

3.2 L'évaluation formative et le cadre cognitif .......................................................... 74

3.2.1 Le renforcement formatif dans une approche cognitive renouvelée ........................................................................................................................... 75

3.2.2 La production écrite, une activité d'exercice et d'apprentissage de stratégies cognitives

3.3 Principes de correction formative de la production écrite

Annexe I Guide de correction référentielle et interactive.

Annexe II Grille «A» de base (Boyer, Roy et Roy, 1991).

Annexe III Grille «B» finale.

Annexe IV Exemples d'exercices correctifs pour les catégories 400, 500 et 700.

Annexe V Portrait diagnostique de groupe.

Annexe VI Portrait diagnostique individuel. 


\section{LISTE DES TABLEAUX}

\section{Tableau}

1 Résultats du test «Repérage d'erreurs»

2 Résultats de l'étude comparative sur l'orthographe d'élèves québécois ... 


\section{LISTE DES FIGURES}

\section{Figure}

1 Grille d'évaluation d'un texte argumentatif du MEQ …...................................... 14

$2 \quad$ Élaboration des objectifs spécifiques de la recherche ……….............................. 27

3 Catégories de la grille «A» de base …………………................................................. 31

4 Catégories de la grille «B» finale ………….................................................................. 34

$5 \quad$ Sous-catégories de la catégorie 100: Orthographe d'usage …............................ 38

6 Sous-catégories de la catégorie 200: Morphologie …......................................... 42

7 Exemples d'erreurs à classer à l'intérieur de la sous-catégorie 210: Morphologie du nom

8 Exemples d'erreurs à classer à l'intérieur de la sous-catégorie 220: Morphologie des adjectifs qualificatifs

9 Exemples d'erreurs à classer à l'intérieur de la sous-catégorie 230: Morphologie des articles et des adjectifs déterminatifs

10 Exemples d'erreurs à classer à l'intérieur des sous-catégories 240: Morphologie du pronom, 260: Morphologie de l'adverbe et 250: Morphologie du verbe

11 Sous-catégories et sous-sous-catégories de la catégorie 300: Accord ........

12 Sous-catégories de la catégorie 400: Structure de la phrase

13 Exemples d'erreurs à classer à l'intérieur de la sous-catégorie 410: 
14 Exemples d'erreurs à classer à l'intérieur de la sous-catégorie 420: Référent

15 Exemples d'erreurs à classer à l'intérieur de la sous-catégorie 440: Mot superflu

16 Sous catégories et sous-sous-catégories de la catégorie 500: Signes de ponctuation et conventions

17 Exemples d'erreurs à classer à l'intérieur des sous-sous-catégories 521:

Virgule de reprise et 522: Virgule d'explication

18 Exemples d'erreurs à classer à l'intérieur de la sous-sous-catégorie 523:

Virgule d'inversion

19 Exemples d'erreurs à classer à l'intérieur de la sous-sous-catégorie 524: Virgule d'ellipse et de retrait

20 Sous-catégories de la catégorie 600: Lexique

21 Exemples d'erreurs à classer à l'intérieur de la sous-catégorie 610: Impropriété

22 Exemples d'erreurs à classer à l'intérieur de la sous-catégorie 620: Accord conceptuel

23 Exemples d'erreurs à classer à l'intérieur de la sous-catégorie 640: Barbarisme, pléonasme et tautologie

24 Exemples d'erreurs à classer à l'intérieur de la sous-catégorie 650:

Niveau de langue

25 Sous-catégories de la catégorie 700: Stylistique

26 Organisation en triptyque de la catégorie 700: Stylistique

27 Organisation en triptyque de la catégorie 800: Qualité textuelle

28 Sous-catégories et sous-sous-catégories de la catégorie 800: Qualité textuelle

29 Sous-catégories de la catégorie 900: Qualité de la pensée

30 Organisation en triptyque de la catégorie 900: Qualité de la pensée 
31 Processus et événements d'enseignement-apprentissage d'une règle en habileté intellectuelle

32 Schéma trois dimensions des habiletés cognitives ………….................................. 78

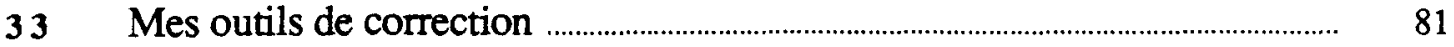

34 Principes de correction formative …………............................................................... 83 
INTRODUCTION 
Les résultats d'une étude menée en 1995 par le professeur Gagné, pour le Conseil de la langue française et le ministère de l'Éducation, font voir que les étudiants de troisième secondaire du Québec ne réussissent qu'avec une moyenne de 64,4 p. cent un test de niveau en grammaire et orthographe. Des résultats de cet ordre ne sont pas nouveaux. Un mouvement de recherche amorcé au début des années '70 a livré de multiples études offrant des résultats semblables que ce soit pour le niveau secondaire, le niveau collégial ou le niveau universitaire. Ce mouvement a par ailleurs mené à des recherches visant à concevoir des moyens didactiques d'intervention appropriés.

Les deux mouvements de recherche utilisent des grilles de correction. Bibeau (1975), Martin et al. (1978), Bureau (1985), de même que Roy et Lafontaine (1992) les utilisent comme outil de classement de fautes. Pour d'autres auteurs (Troestler, 1978; Beaulieu et al., 1978; Jean et al., 1988; Des Lauriers et Major, 1992), la grille de correction est vue comme un moyen didactique d'intervention.

L'étude présentée dans ces pages est de la nature d'une recherche-développement. Elle propose une grille renouvelée de correction des productions écrites. C'est en effet un premier trait d'originalité de cette recherche que de présenter une grille comportant des catégories nouvelles de classement auxquelles se greffent certaines sous-catégories moins habituelles. Des exemples et des propos explicatifs s'attachent à justifier, en toutes ses parties, cette grille renouvelée.

Un deuxième trait d'originalité tient au choix et à l'organisation inteme des sous-catégories des trois catégories relevant traditionnellement du «fond», soit de Stylistique, de 
Qualité textuelle et de Qualité de la pensée. En effet, pour ces trois catégories il a été créé un modèle de hiérarchisation se présentant sous la forme d'un triptyque. Ce modèle particulier de hiérarchisation donne une cohésion nouvelle aux parties de ces ensembles que leurs principes, relevant plus de l'art que de la science, condamnent à ne constituer que des inventaires.

Un troisième trait d'originalité de l'étude vient du fait que la grille ait adopté comme cadre d'utilisation l'approche d'évaluation formative. Cette nouveauté a même permis de faire ressortir des principes venant guider l'utilisation de la grille, principes tirés principalement d'une interprétation pragmatique des théories relatives à une approche formative.

Évidemment cet ensemble de nouveautés s'accompagne de limites. Une première limite est celle d'avoir dû disperser le cadre théorique dans l'ensemble des analyses. Il y a là l'effet d'abord de la distribution des données qu'imposent les multiples catégories de la grille, qu'impose également l'apparition tardive des références au modèle formatif.

De plus, l'approfondissement des théories qui servent de fondement à la grille a dû être limité. Étant donné que toutes les notions relatives à la langue sont présentes à l'intérieur d'une grille qui cherche à en couvrir forcément tous les aspects, il devenait impossible de présenter, dans une seule recherche, une analyse exhaustive d'un champ aussi vaste. C'est donc une deuxième limite de l'étude de n'aborder qu'en surface l'examen des différentes catégories et sous-catégories de la grille. Ces choix faits l'ont été dans le but de permettre une familiarisation réelle, pratique et facilitante avec les catégories et les souscatégories de la grille. 
De la nature d'une recherche-développement, cette étude, dans sa partie développement, se devait d'offrir un matériel pratique. Ce matériel prend la forme d'un Guide de correction référentielle et interactive, que le lecteur trouvera d'ailleurs en annexe. Ce guide présente de façon pratique, et dans une disposition de consultation aisée, les catégories et les sous-catégories qui auront été présentées dans l'analyse du deuxième chapitre. Le mâ̂tre trouvera là un outil indispensable au type de correction proposé dans ces pages. Le maître trouvera d'ailleurs dans les toute premières pages du guide les principes de correction formative du dernier chapitre, à la suite des règles d'utilisation de la grille.

Le chapitre premier inventoriera les recherches traitant des problèmes et des préoccupations relatives aux productions écrites; il se terminera sur la présentation des objectifs de la recherche. Le deuxième chapitre, pour sa part, présentera l'outil que constitue la grille de correction, en en détaillant les composantes; il montrera aussi les principaux fondements de l'analyse. Finalement, dans le troisième et dernier chapitre de l'étude, il sera traité du cadre d'approche formative, cadre considéré comme étant l'environnement pédagogique et didactique hors duquel l'utilisation de la grille perdrait l'essentiel de ses effets bénéfiques; le chapitre se terminera par la présentation d'un certain nombre de principes de correction formative tirés de l'interprétation pragmatique de propos portant sur le cadre formatif des productions écrites. 


\section{CHAPITRE PREMIER}

Les travaux de recherche menés sur le thème

$$
\text { de la maîtrise de l'écriture }
$$


La maîtrise du français écrit des élèves du collégial fait régulièrement l'objet d'épreuves d'évaluation émanant du ministère de l'Éducation du Québec. Au collégial, les étudiants doivent se soumettre à un test de français écrit administré par la Direction générale de l'enseignement collégial, test permettant aux candidats de passer du niveau collégial au niveau universitaire. Les résultats sont régulièrement déplorables et indiquent un faible niveau de maîtrise, ne dépassant pas, pour souvent la moitié des candidats, la note de passage fixée à $60 \%$. Par ailleurs, le niveau de maîtrise ne se révèle pas meilleur au secondaire et au primaire. La situation est inquiétante: les écoliers éprouvent des difficultés d'écriture et ce, à plusieurs niveaux.

Ce n'est pas d'hier que l'intérêt se tourne vers ce problème. Nombreuses sont les études qui visent à mesurer les difficultés d'écriture des écoliers et qui cherchent à élaborer des moyens d'intervention menant à l'amélioration du niveau de maîtrise du français écrit. L'examen de ces travaux permettra, dans un second temps, de préciser l'objet, l'orientation et la méthodologie de la recherche présentée dans ces pages.

\subsection{LES TENDANCES MARQUANTES DES ÉTUDES MENÉES DANS LES VINGT DERNIÈRES ANNÉES}

Même si les études dépouillées ont permis d'identifier des divergences dans les orientations de recherche, trois tendances se dessinent nettement dans les travaux qui se sont intéressés, depuis les années '70, à la qualité du français écrit dans les écoles et les universités. La recension permettra de situer les grandes voies de recherche, de faire voir les intentions des projets et de présenter les traitements administrés. 
Une première tendance se retrouve dans les travaux qui se proposaient de mesurer le niveau global de maîtrise du français écrit et forcément de présenter des analyses des erreurs commises. Bibeau (1975), Martin et al. (1978), Roy et Lafontaine (1992), Roberge (1984), Bureau (1985) et le M.E.Q. (1994: voir Lépine, 1995), entre autres, ont cherché avant tout à mesurer l'ampleur et la nature des difficultés des élèves.

Une deuxième tendance regroupe les travaux, souvent des maitres en pratique, portant sur les moyens d'amélioration du niveau de maîtrise du français écrit. Cette tendance «pédagogique» englobe les travaux des chercheurs suivants: Troestler (1978), Beaulieu et al. (1977; 1978), Jean et al. (1988) et Gagnon (1993), entre autres.

Finalement, une troisième tendance se retrouve dans les travaux des chercheurs et des enseignants qui proposent une approche d'évaluation formative en vue de faire progresser le scripteur dans ses apprentissages de la langue écrite. Ces travaux plus récents sont le fait de Des Lauriers et Major (1992), Gagné (1986) et Roberge (1993), entre autres.

\subsubsection{Première tendance: la mesure stricte du niveau de maîtrise du fran- çais écrit}

Le choix fait par les chercheurs de cette tendance était de mesurer le niveau global de maîtrise du français écrit à des niveaux d'ordres scolaires différents et ce, afin de présenter un portrait des difficultés des élèves. Au tournant des années soixante-dix, une dégringolade de la maitrise du français écrit chez les élèves fut remarquée par les maitres. Les chercheurs désiraient donc quantifier cette situation et rendre disponibles des portraits statistiques de la situation. Ils ont donc opté pour des mesures strictes d'évaluation du niveau de maîtrise du français écrit. 
À l'époque, l'évaluation de la maîtrise en français écrit dans les établissements collégiaux attirait l'attention. La formation supposément terminée, il était constaté que la mâ̂trise du français écrit restait insuffisante. En 1975, Bibeau, dans son Enquête sur le français écrit dans les cégeps a mené une étude à grande portée, il a dressé un portrait d'ensemble des difficultés en français écrit des étudiants des 21 cégeps du Québec. Sur la base d'un échantillon aléatoire, 2385 candidats ont produit un texte de 250 mots sur un sujet d'intérêt général; puis leurs écrits ont été corrigés à l'aide de quatre grilles de correction: Générale, Orthographe flexionnelle et de morphologie, Orthographe, Ponctuation. De plus, quelques critères étaient considérés, tels la population, le sexe, la répartition régionale, le secteur, la concentration, l'année d'étude et finalement la profession des parents.

Cette étude a montré qu'en 1975 un collégien faisait en moyenne une faute à tous les 19 mots et que ces fautes étaient généralement des fautes de virgules, d'indicatif présent, de nom commun et d'épithète. De plus, selon des analyses complémentaires, les filles faisaient moins de fautes que les garçons, les étudiants dont les parents étaient scolarisés réussissaient mieux et les étudiants du secteur professionnel éprouvaient plus de difficulté.

À l'époque, cette recherche fit prendre conscience, en quelque sorte, du faible niveau de maitrise en français écrit de cette génération d'élèves. Nombreuses furent les retombées de cette étude dans le milieu des chercheurs intéressés par le sujet. Elle ouvrait des portes et même de nouvelles perspectives d'analyse.

Martin et al. (1978) se sont aussi intéressés à la qualité du français au collégial, mais cette fois au français oral des étudiants. Les auteurs ont mené, en collaboration avec la Direction générale de l'enseignement collégial, une recherche intitulée: L'évaluation de la 
compétence linguistique du vocabulaire actif des étudiants de première session au niveau collégial, dans le but, d'une part, d'évaluer le vocabulaire et les connaissances linguistiques et, d'autre part, de comparer le niveau de maîtrise des candidats selon leur provenance: milieu rural ou milieu métropolitain. Deux groupes, représentant les deux milieux, étaient à l'étude. La cueillette de données fut réalisée à l'aide d'entretiens enregistrés sur bandes magnétiques. Les critères d'évaluation allaient d'un jugement très favorable à un jugement très défavorable; ils référaient à la clarté, à la concision, à la connaissance du sujet, à la compétence générale en français, au vocabulaire, à la syntaxe, à la prononciation et à la structure du discours.

Les résultats pour les deux groupes sont sensiblement les mêmes, quoiqu'en général, diront les auteurs, le niveau de langue d'un étudiant provenant d'un milieu métropolitain, comparativement à celui issu d'un milieu rural, est plus satisfaisant. Les catégories d'erreurs les plus «populaires» sont celles touchant le vocabulaire, la syntaxe et le manque de précision dans le discours. Toutefois, le vocabulaire général est convenable, ainsi que la prononciation et la cohérence du langage.

Cette étude s'est intéressée de près au niveau d'expression orale des jeunes collégiens et a permis d'importantes analyses. Toutefois, ces dernières se basent uniquement sur des jugements d'appréciation globale et ne ciblent pas suffisamment les difficultés.

Pour sa part, le chercheur Roy est allé plus loin dans ses analyses. Intéressé davantage au niveau universitaire, cet auteur a mené, au cours des dernières années, plusieurs études spécialisées et diagnostiques sur l'état du français écrit. Une étude qui a retenu l'attention est celle réalisée conjointement avec Lafontaine (Roy et Lafontaine, 1992), ayant pour titre: Étude de la mâttrise du français écrit à l'Université. Cette étude avait comme 
objectif de transmettre un diagnostic des connaissances en français écrit de la clientèle universitaire, puis de comparer leur maîtrise selon plusieurs caractéristiques d'ensemble. L'ampleur de cette recherche oblige à ne traiter que des résultats obtenus.

Les candidats à l'étude, c'est-à-dire tous les arrivants à l'Université de Sherbrooke en 1987, devaient se soumettre à trois types de tests. Le premier test «Repérage d'erreurs» a enregistré de faibles taux de réussite pour toutes les catégories retenues, ce que laisse voir le tableau 1. Pour sa part, le deuxième test «Correction de texte» a permis d'identifier la concentration la plus forte en français écrit: Médecine; et la plus faible: Éducation physique et sportive. Finalement, le troisième test «Rédaction» a enregistré un taux de réussite de $50,78 \%$.

TABLEAU 1

Résultats du test krepérage d'erreurs
\begin{tabular}{|lc|}
\hline Syntaxe & (Roy, \\
Vocabulaire & $50,1 \%$ \\
Orthographe grammaticale & $49,9 \%$ \\
Accord des verbes & $53,3 \%$ \\
Accord des participes passés & $47,6 \%$ \\
Accord de genre et de nombre & $45,1 \%$ \\
Orthographe d'usage & $45,2 \%$ \\
\hline
\end{tabular}

D'autres données ressortent des analyses de Roy et de Lafontaine: les filles réussissent mieux que les garçons, les étudiants en Sciences obtiennent de meilleurs résultats, de même que les étudiants les plus âgés. De plus, l'étude a permis de montrer qu'il y avait, et heureusement, une progression d'un niveau à l'autre. Ainsi, une amélioration de 19,84\% du secondaire à l'Université est apparue, puis une de 4,55\% après trois ans d'université. 
La recherche réalisée par Roy et Lafontaine a analysé le niveau de maîtrise de français écrit à l'université sous pratiquement tous ses angles. Les analyses poussées des auteurs de l'étude ont de ce fait permis l'élaboration de portraits précis sur le niveau de maîtrise en français écrit des étudiants universitaires. Toutefois, les résultats touchant la progression des différents niveaux scolaires (secondaire et universitaire) ne semblent pas convaincants étant donné l'utilisation d'échantillons hétérogènes. Il faudrait plutôt parler d'une comparaison entre les niveaux de l'époque plutôt que d'une progression.

Après s'être intéressés de près aux niveaux post-secondaires, certains auteurs ont cru bon, pour leur part, de vérifier s'il y avait aussi une dégradation pour les élèves de première secondaire, entre autres. Roberge (1984) dans son Étude comparative sur l'orthographe d'élèves québécois a mené une étude sur l'état de la maîtrise des élèves du début du secondaire. L'expérience qu'il a tentée avait pour but de comparer le niveau de maitrise du français écrit des élèves de niveau secondaire de la génération présente - celle de 1982 - à celle d'il y a vingt ans. Les groupes expérimentaux étaient constitués, d'une part, de 426 copies d'un test d'élèves de septième année réalisé en 1961 pour l'obtention du certificat d'études primaires des écoles de la Commission catholique de Sherbrooke et, d'autre part, de 348 élèves de première secondaire, inscrits à l'école St-François Xavier de l'Estrie, en 1982. Ces demiers devaient transcrire un texte dicté à voix haute qui était identique à celui de 1961. Les cinq catégories de classement des fautes étaient les verbes, les noms; les adjectifs, les pronoms et les mots invariables. Par la suite, un sous-classement des erreurs était réalisé relativement à la non-application d'une règle de grammaire, à la non-conformité de l'usage proposé par le dictionnaire et finalement au cumul de ces deux sous-classes. 
L'analyse de Roberge a ainsi permis de montrer que les erreurs réalisées par les élèves de 1982 étaient de plus grande envergure que celle des élèves de 1961. Les résultats des erreurs commises pour une dictée de 120 mots pour les deux groupes sont inscrits à l'intérieur du tableau 2. Ce tableau montre aussi que les filles réussissent mieux que les garçons.

TABLEAU 2

Résultats de l'Étude comparative sur l'orthographe d'élèves québécois (Roberge, 1984).

\begin{tabular}{|l|c|c|c|c|}
\hline & Garcons & Garcons & Filles & Filles \\
\hline Groupes & I (1961) & II (1982) & I (1961) & II (1982) \\
\hline Nombre de sujets & 222 & $\mathbf{1 6 7}$ & 204 & $\mathbf{1 8 1}$ \\
\hline Nombre d'erreurs & 2,3 & $\mathbf{1 8 , 8 3}$ & 0,02 & $\mathbf{1 4 , 2 7}$ \\
\hline
\end{tabular}

Les résultats obtenus parlent d'eux-mêmes. Étant donné qu'en 1962, l'insistance portait beaucoup moins sur l'évaluation du français écrit, l'auteur cherchait à savoir s'il y avait réellement un écart important et ce, comparativement à 1982. La recherche s'est vue alors transmettre d'importantes informations et un cri d'alarme, encore une fois, à la population scolaire. Toutefois, l'outil de classement utilisé pour la recherche est quelque peu faible. Même si l'objectif n'en était pas un de classification, il aurait été avantageux de cibler avec plus d'exactitude les difficultés des élèves à l'étude.

Le professeur Bureau (1985) est un autre chercheur qui s'est intéressé en profondeur au sujet et qui a fourni des analyses précises des erreurs commises, aussi, par les élèves de niveau secondaire. C'est ainsi qu'il a produit autour des années ' 80 plusieurs études de type statistique. Une, entre autres, intitulée: Le français écrit au secondaire a permis de tracer des portraits précis sur l'état de la maîtrise du français écrit des élèves de ce 
niveau et de produire des analyses diagnostiques accompagnées de recommandations de type didactique.

Les 353 candidats âgés de 13 à 17 ans de quatre écoles de la Commission des écoles catholiques de Québec devaient composer un texte de 20 lignes sur un sujet libre. La grille utilisée comprenait les catégories: Grammaire, Syntaxe, Sémantique, Orthographe, Morphologie, Anglicisme, Code écrit et Inattention. Les résultats indiquent que les élèves du secondaire font en moyenne 37 erreurs par copie. Toutefois, cette moyenne varie selon le niveau de l'étudiant, passant de 60 fautes en première secondaire à 25 fautes en cinquième secondaire. Les catégories responsables du plus haut taux d'erreurs commises sont celles de la grammaire $(35,8 \%)$ et de l'orthographe $(39,1 \%)$.

Par la suite Bureau, relativement aux résultats obtenus, a tenté des «implications pédagogiques»; autrement dit des réflexions ou des pistes utiles pour l'enseignement du français. Ces pistes font montre d'une analyse poussée des différentes catégories de fautes. Par exemple, Bureau (1985) explique qu'il faut corriger les difficultés principalement dans l'accord des noms, s'attaquer davantage aux régularités du système (plutôt qu'aux exceptions), vérifier si les accords réguliers sont acquis et traiter de l'accord des mots les plus fréquents.

Il ne fait pas de doute que les travaux de Bureau ont marqué le milieu de la recherche et ont, à ce qu'il semble, influencé certaines des pratiques officielles d'évaluation et, par ricochet, la construction des épreuves ministérielles. Ces épreuves ministérielles font d'ailleurs souvent l'objet de vives critiques de la part de la communauté scientifique.

À ce propos, l'épreuve unique d'évaluation de la compétence langagière du M.E.Q., à laquelle doivent se soumettre depuis quelques années les étudiants du collège, consiste à 
rédiger un texte argumentatif de 500 mots sur l'un des trois thèmes présentés. La figure 1 fait état des catégories de la grille utilisée par le M.E.Q. (1994: voir Lépine, 1995), laquelle compte sept éléments: quatre pour le discours et trois pour la langue. L'étudiant échoue s'il commet plus de 20 fautes pour les deux derniers critères. Il semble donc que la correction en soit majoritairement une de comptabilisation des fautes et non une de vérification de la mâtrise globale du candidat.

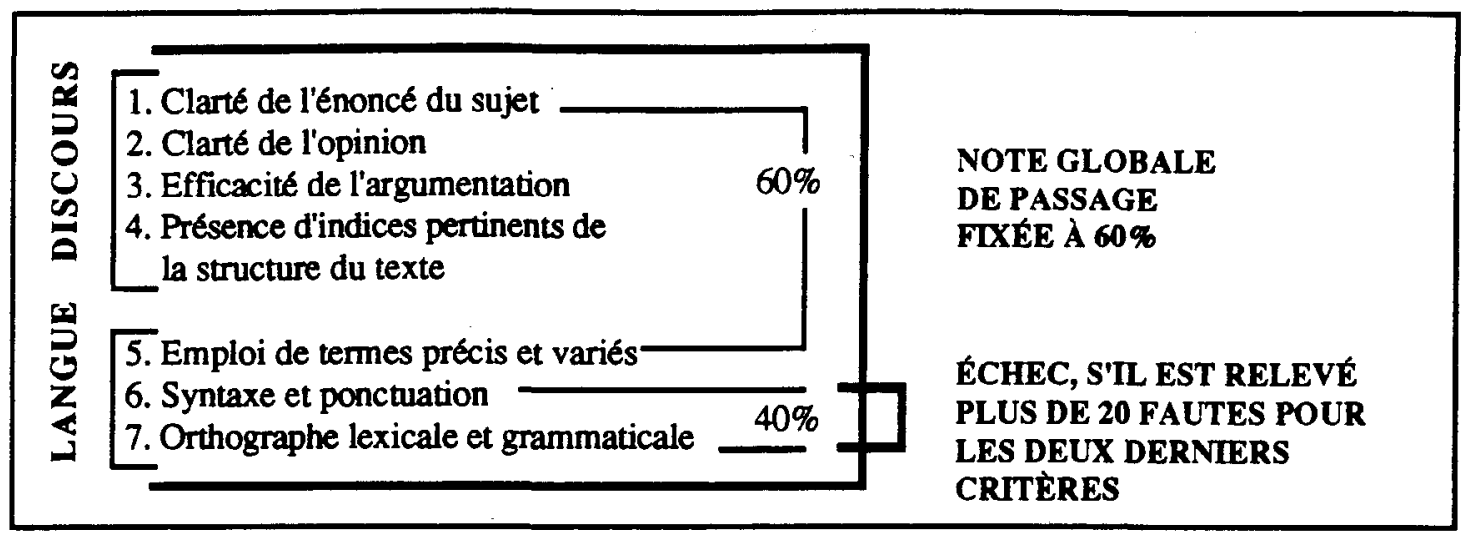

Figure 1: Grille d'évaluation d'un texte argumentatif du M.E.Q. (1994: voir Lépine, 1995).

De plus, Lépine (1995, p.24) dans son article intitulé: Bilan des tests de français à l'admission aux universités québécoises (1987-1994) de la Revue des sciences de l'éducation indique que «la pondération des critères amène à penser que [les] auteurs ont voulu mettre l'accent plutôt sur l'aptitude à construire un discours clair et cohérent que sur la maitrise de l'orthographe et de la grammaire: les critères 1 à 5 totalisent $60 \%$ de la note globale». Lépine se questionne donc sur ce que veulent mesurer en réalité les épreuves du M.E.Q.. De plus, il croit que «la valeur indicative de l'examen du ministère de l'Éducation est faible, voire dommageable sur le plan didactique, car elle ne peut vraiment servir de guide dans l'élaboration de mesures correctives touchant le discours et destinées aux étudiants qui ont échoué (Lépine, 1995, p.28)». Il semblerait donc que le but du ministère ne soit pas d'indiquer honnêtement le degré de difficulté, mais bien de sanctionner les 
candidats. L'outil, d'ailleurs très peu détaillé, ne peut en aucun cas informer le scripteur de ses difficultés et ne peut surtout pas être utile aux maîtres dans les activités de préparation au test.

C'est d'ailleurs ce qui est souvent reproché par les enseignants. Ces derniers veulent préparer et aider leurs élèves en difficulté aux différents tests du ministère. Les enseignants ne disposent alors comme unique outil que de la grille de correction du M.E.Q., laquelle, encore une fois, ne sert qu'à noter les copies. Les maîtres, ceux qui veulent utiliser des outils autres que ceux du M.E.Q., doivent développer leurs propres méthodes. Ces méthodes, regroupées à l'intérieur des recherches de la deuxième tendance, seront illustrées à l'intérieur de la prochaine partie.

Un certain nombre d'études de la première tendance ont cherché à fournir des diagnostics précis sur la maîtrise du français écrit. Ces études très «statistiques» ont permis de percevoir l'ampleur du problème et de transmettre des portraits diagnostiques globaux des difficultés. Toutefois, les résultats fournis révèlent souvent des difficultés tellement généralisées et étendues qu'il devient difficile d'en tirer un ou des diagnostics non équivoques. Chaque portrait differe et ce, selon l'outil de classification utilisé, donc des divisions de la grille servant d'outil.

Pour sa part, la deuxième tendance identifiée est celle regroupant des études réalisées par des maîtres en pratique. Les travaux se veulent très pédagogiques et pragmatiques. Les maîtres, soucieux de la réussite de leurs étudiants, ont perçu la situation et tentent selon leur expérience, leur intuition pratique et leur conviction d'intervenir à l'aide de méthodes dites pédagogiques. 


\subsubsection{Deuxième tendance: les recherches sur l'intervention en maîtrise du français écrit}

Les enseignants, en salle de classe, cherchent comment s'y prendre pour que leurs apprenants progressent en écriture. Ils se demandent sur quoi faire porter leurs efforts, quelles méthodes utiliser pour améliorer la situation; car l'école, il y a consensus, doit mener les jeunes Québécois qui la fréquentent à un seuil satisfaisant de réussite en français écrit.

C'est en 1978 que le professeur Troestler, dans son Essai de typologie des fautes, a fait connaître les résultats d'une recherche-milieu présentant une audacieuse typologie des erreurs de ses étudiantes inscrites en secrétariat, élaborée dans le cadre d'intérêts d'applications pédagogiques. La tâche des candidates consistait à produire du texte, puis à cibler leurs erreurs pour enfin les corriger à l'aide des moyens mis à leur disposition, entre autres les interventions du professeur. Les candidates faisaient par la suite une auto-évaluation, prenaient conscience de leurs difficultés et tentaient de trouver un moyen pour ne plus répéter leurs erreurs. Selon Troestler (1978), les interventions ont occasionné une diminution appréciable des fautes et la méthode utilisée a permis aux étudiants d'éliminer certains types d'erreurs. Les travaux de cet auteur sont donc pragmatiques et les moyens didactiques proposés étaient fort originaux, bien que d'une complexité au premier abord déconcertante.

Les membres de l'équipe RIEFEC du collège Bois-de-Boulogne, soit Beaulieu, Bourgeau et Paquin (1977), dans leur étude ayant pour titre Les erreurs les plus fréquentes en français écrit au collégial: les participes passés et les formes adjectivales: description des comportements déviants, matériel didactique correctif, tentaient à l'époque une recherche nouveau genre. Les auteurs voulaient, de fait, décrire les comportements relatifs aux 
erreurs réalisées le plus souvent en français écrit, puis, pour ces erreurs, élaborer du matériel correctif. À vrai dire, ils cherchaient à donner «le pourquoi» des erreurs commises.

Après avoir déterminé les fautes les plus fréquemment commises retrouvées à l'intérieur des 2385 copies d'étudiants de première année inscrits au Cégep de Maisonneuve, les auteurs ressortaient de leurs analyses que les fautes des étudiants étaient attribuables aux composantes syntagmatiques, à l'influence de l'oral et à la logique du scripteur. Ainsi, pour élaborer leur matériel correctif, les auteurs ont tenu compte des résultats et se sont inspirés du guide méthodologique de J. G. Milot. Finalement, ils ont opté pour une méthode par induction: d'abord l'observation, ensuite la formulation de la règle par l'étudiant et finalement l'application des connaissances.

Les intentions de cette recherche étaient de taille. L'étude a tenté d'abord d'identifier les difficultés, mais a surtout consisté à produire du matériel correctif. Pour ce matériel, la forme inductive adoptée est apparue appropriée, car elle visait à faire réfléchir la langue par l'apprenant. Par contre, la qualité du procédé ayant pour but d'expliquer les erreurs commises par les candidats est apparue quelque peu discutable. Malgré tout, certaines de ces explications ont permis d'élaborer le matériel et de comprendre un peu mieux les difficultés.

En 1978, les mêmes auteurs: Beaulieu, Bourgeau et Paquin proposaient, cette foisci, un guide méthodologique intitulé: Consolidation des acquis en français écrit au collégial. Ce matériel, élaboré dans des orientations semblables à celles de la précédente étude, proposait des moyens didactiques divers. Les auteurs de ce guide méthodologique suggéraient l'utilisation d'une grille de correction remise aux élèves et ce, dans le but de les amener à une plus grande prise en charge de leurs apprentissages. Les chercheurs justifiaient aussi leur approche parce qu'elle permettait aux maîtres de cibler plus facilement les diffi- 
cultés pour pouvoir y travailler par la suite avec plus d'intérêt. Les propositions pédagogiques des auteurs ne se sont pas limitées à celle d'utiliser une grille, mais aussi et entre autres, à des méthodes, telles que le contrat, la fiche d'observation, la grille de relecture, le didactexte et les ateliers de dépannage.

En plus de proposer tout un amalgame d'outils d'intervention, les membres de cette équipe ont mené une réflexion sur l'activité qu'est de corriger. Selon eux, il importe que l'activité de correction mène à des apprentissages, qu'elle développe une prise de conscience de la part des apprenants et qu'elle fasse naître certains mécanismes chez eux. Beaulieu, Bourgeau et Paquin (1978) soulignent aussi l'importance de l'implication du maître dans la correction, soit encadrer, écouter, puis enseigner selon les difficultés perçues.

Les préoccupations de ces auteurs sont donc aussi très pédagogiques et ont pour objet immédiat une augmentation, une amélioration de la maîtrise des élèves par une spécialisation des moyens didactiques d'intervention. Quoique la grille proposée soit quelque peu complexe, les initiatives et les réflexions proposées dans cette étude sont de grande qualité. Ces travaux ont d'ailleurs été bien publicisés et ont influencé beaucoup de maîtres.

Les enseignants Jean, Leclerc et Racine (1988), aussi du collège Bois-de-Boulogne, proposaient à cette époque La grille de correction du S.A.L.F.. Cette grille comptait 27 éléments répartis à l'intérieur de cinq grandes catégories: Orthographe, Grammaire, Phrase, Vocabulaire et Texte. Cette grille poursuivait comme objectifs principaux de mener les étudiants du collège à améliorer la qualité de leur français écrit et de les rendre plus autonomes dans leur démarche de correction. L'approche pédagogique privilégiée insiste sur une démarche d'autocorrection. À l'aide de la «Fiche d'analyse des erreurs», inspirée de la 
méthode RIEFEC, l'étudiant devait inscrire la cote correspondant à la faute, puis la correction et la règle relative à l'erreur. Cette méthode permettait à l'étudiant de mieux mettre en relief ses principales difficultés qu'il tentait par la suite de faire disparaitre.

Tout compte fait, le collège Bois-de-Boulogne est allé loin dans ses démarches de recherche et d'applications pédagogiques. Toutefois, la grille utilisée et proposée gagnerait à contenir des éléments plus nets qui pourraient être davantage des pistes d'apprentissage pour les scripteurs. Une catégorie du type «Homophones», par exemple, ne montre pas réellement la nature de la faute et la piste des apprentissages possibles à faire faire par le scripteur. Malgré tout, il semble que la grille ait été un bon outil d'intervention et ait amené les étudiants du collège Bois-de-Boulogne à progresser en français.

Le ministère de l'Éducation du Québec avait aussi, quoi que certains en pensent, des préoccupations d'applications pédagogiques. Gagnon (1993), responsable à l'époque de l'évaluation des apprentissages en français au ministère de l'Éducation, publiait dans la revue Québec français (automne 1993) un article intitulé: «Un instrument diagnostique en écriture». L'utilisation de cet outil poursuivait comme objectif d'identifier la nature des difficultés et ce, en prévision d'une intervention efficace. L'épreuve était constituée de deux parties indissociables, soit «Les connaissances» et «Le transfert des connaissances». La première partie voulait cerner les connaissances acquises des élèves du secondaire en posant des questions relatives à plusieurs règles de grammaire. La deuxième partie, pour sa part, consistait à faire rédiger un texte permettant d'évaluer la capacité de l'élève à faire le transfert de ses connaissances en situation d'écriture.

Le ministère de l'Éducation semblait enfin se tourner vers une approche diagnostique axée sur la progression, la poursuite et l'amélioration du français écrit. Toutefois, malgré les bonnes intentions du ministère, la première partie, qui permet de diagnostiquer 
les difficultés sous forme de questions, n'apparâ̂t pas pouvoir faire nettement son travail étant donné la complexité des questions posées. L'épreuve apparaît quelque peu irréaliste en vue des objectifs à atteindre. Elle devait d'ailleurs être testée en 1994, mais ce projet a dû être abandonné en raison, semble-t-il, de coupures de budgets gouvernementaux.

Les recherches des auteurs regroupés à l'intérieur de la deuxième tendance ont manifesté des soucis pratiques d'application et d'intervention auprès d'une clientèle en difficulté d'écriture. Les moyens d'intervention utilisés l'ont été de toutes sortes. La plupart du temps, il s'agissait de faire retravailler le texte. Des pratiques d'autocorrection étaient aussi suggérées et ce, dans le but de faire réfléchir sur les structures de la langue. Plusieurs outils ont été proposés, entre autres une grille de correction. Cette méthode était justifiée pour sa portée diagnostique et pour son utilité de référence à l'erreur.

La prochaine tendance s'oriente lentement vers des méthodes davantage axées sur la prise de conscience. Les maîtres désirent intervenir, mais désirent que leurs interventions mènent à des prises de conscience, donc à des apprentissages construits. De plus, l'apprenant et son rythme d'apprentissage sont respectés davantage, c'est-à-dire que le cheminement de l'individu engagé dans son apprentissage de l'écriture est considéré. La troisième tendance présente les recherches ayant adopté, comme méthode d'intervention, l'approche formative de correction.

\subsubsection{Troisième tendance: les recherches sur l'intervention en approche formative}

Même si l'évaluation formative date déjà d'une vingtaine d'années et plus, certains travaux lui donnent plus récemment une place d'importance dans la mise en application de moyens didactiques d'intervention. Les recherches de cette tendance adhèrent à ce type 
d'intervention parce qu'il est axé principalement sur l'apprenant, sur son cheminement et sur sa progression.

Tout récemment Des Lauriers et Major (1992) de la Commission scolaire SainteCroix ont conçu un code de correction pour le français au secondaire: Cent fautes ou sans faute. L'utilisation de ce code poursuivait principalement de venir en aide aux scripteurs lors de la rédaction de leur texte en français. Le code est en quelque sorte une grille utile aux maîtres afin de corriger et aussi afin de constater les progrès de leurs élèves. Pour faire l'élaboration de cet outil, les auteurs ont relevé les erreurs les plus fréquentes présentes dans les copies d'élèves du secondaire. Quatre catégories de référence ont ainsi été déterminées: Lexique, Grammaire, Syntaxe et Composition. Plusieurs autres sous-catégories et sous-sous-catégories s'y rattachent.

Les auteurs proposaient aussi plusieurs stratégies d'application du code dont l'une, qui intéresse particulièrement, appelée «La production écrite formative». Cette stratégie se base sur une autocorrection de la part de l'élève. Après avoir rédigé son texte, l'élève est amené à faire une révision de son écrit et ce, aidé du code de correction et des ouvrages de référence disponibles. Par la suite, l'enseignant corrige la copie de l'élève à l'aide du code et l'encourage à se corriger et à inscrire la règle appropriée à l'intérieur du «Tableau de correction pour l'élève», pour ensuite dresser son portrait personnel à l'aide du «Tableau de compilation». Selon les auteurs, cette pratique encourage l'élève à s'autocorriger et à s'efforcer de ne plus répéter les mêmes erreurs.

Les enseignants Des Lauriers et Major appuient une démarche formative parce qu'elle mise d'abord sur la progression du scripteur. Selon eux, en indiquant constamment à l'élève ses difficultés, puis en lui offrant des moyens appropriés pour les contrer, les chances de succès se multiplient. Ces auteurs se dirigent donc vers la voie de l'avenir, 
c'est-à-dire vers une approche davantage diagnostique, davantage axée sur le cheminement personnel de l'apprenant. Toutefois, le fait d'avoir construit le guide en se basant sur les difficultés les plus fréquentes n'apparaît pas pouvoir couvrir tout le champ des difficultés possibles en écriture. Le code gagnerait à subir quelques améliorations, surtout dans l'élaboration de certaines catégories et sous-catégories.

Pour sa part Gagné (1986), à l'époque étudiante à la maîtrise en Éducation à l'Université du Québec à Rimouski, croyait aussi en une approche du type formatif et son mémoire de maîtrise a pour titre: L'évaluation formative, la qualité du français écrit et l'attitude des étudiants à l'égard de l'évaluation. Même si ses expérimentations, auprès de deux groupes témoins et d'un groupe expérimental de niveau collégial, n'ont pas pu faire nettement la preuve des bienfaits de l'évaluation formative, l'auteure reste convaincue du rôle prédominant que peut apporter une démarche de ce type sur l'apprentissage des règles d'écriture. Pour Gagné, l'évaluation formative était une modalité d'évaluation respectueuse du rythme de chaque étudiant.

Les interventions formatives de Gagné, entre le prétest et le post-test auprès du groupe expérimental, étaient d'évaluer l'effort et l'amélioration des élèves. Contrairement aux groupes témoins, où une simple note était émise et sans possibilité d'y revenir, les étudiants du groupe expérimental pouvaient corriger les fautes relevées sur la copie, aidés soit de l'enseignante, soit des dictionnaires ou des grammaires. Les étudiants, en plus de corriger leurs fautes, devaient en préciser les natures, ainsi que les règles correspondantes. Aucun point n'était enlevé pour une faute corrigée. Cette pratique avait pour but une réelle prise en charge. Le tout dans un climat d'encouragement et d'entraide.

$\mathrm{Si}$ aucun résultat convaincant ne fut enregistré, il est probable que ce fut causé par les choix expérimentaux de la chercheuse. Cette dernière indique d'ailleurs que ses grou- 
pes, ses instruments de mesure et ses moyens d'intervention auraient gagné à être mieux choisis. De plus, l'application de l'évaluation formative n'est pas tout à fait nette dans cette étude. Il aurait été souhaitable de faire davantage la preuve des lois ou des règles régies par l'évaluation formative. Toutefois, l'attitude des étudiants du groupe expérimental à l'égard de l'évaluation s'est améliorée. Les étudiants la perçoivent maintenant davantage comme une activité aidante en soi.

Une autre chercheuse, Roberge (1993), a aussi changé la perception qu'avaient ses élèves face à l'évaluation. Dans son article intitulé: «Une expérience d'évaluation formative individualisée», paru dans la revue Québec français, il est décrit une expérimentation de correction formative en français écrit. Dans le but de préparer ses élèves à l'épreuve du M.E.Q., Roberge (1993) ne croyait pas qu'une correction traditionnelle pouvait transmettre adéquatement des pistes de correction et d'amélioration pour la réussite de l'examen.

Ainsi, 60 élèves de cinquième secondaire ont profité d'une correction formative orale et individualisée. Les 60 copies étaient minutieusement lues à voix haute, puis corrigées et commentées sur une cassette audio. L'enseignante apportait ainsi ses corrections d'une façon directe et précise à son apprenant. Ce dernier était informé autant du point de vue de l'orthographe, de la syntaxe, de la ponctuation que du point de vue des idées. De plus, l'enseignante tenait compte du rythme d'apprentissage et suggérait des pistes selon le niveau de chacun. Elle prenait aussi le soin de préciser les points forts et faibles de la copie. Selon l'auteure, les élèves ont adoré cette expérience et ils s'empressaient d'écouter les commentaires et surtout de découvrir qu'elles étaient leurs erreurs. Cette pratique a surtout permis d'encourager les élèves à s'efforcer davantage et à ne plus répéter les erreurs lors de l'épreuve officielle du M.E.Q.. 
L'évaluation formative a comme souci d'informer l'étudiant de ses difficultés, puis de le guider dans son cheminement d'apprentissage. L'expérience de Roberge a su respecter les usages à faire de ce type d'évaluation. Toutefois, il aurait été souhaitable que les élèves puissent profiter de cette expérience sur une base continue. Or, cette idée semble quelque peu utopique dans le cadre et l'ampleur du travail qu'exige le type de correction proposée par l'auteure. L'expérience de Roberge (1993) apparaît trop lourde pour la tâche de correction d'un enseignant. De plus, pour bien faire, l'activité aurait dû être complétée d'exercices de révision ou d'enseignements complémentaires référant aux difficultés perçues. De plus, un outil de classification aurait sûrement aidé ou guidé l'enseignante et ses élèves dans leur démarche. La grille du M.E.Q. n'apparaît pas complète en soi pour renvoyer clairement le scripteur à ses difficultés.

Les chercheurs de cette troisième tendance misent donc, dans leurs études, sur l'approche et la méthode d'évaluer pour former. Les auteurs tiennent ainsi compte du cheminement de leurs apprenants en difficulté et mettent en marche ou des méthodes ou des applications pédagogiques pour venir aider leurs apprenants à améliorer la maîtrise de leur français écrit. Or, la plupart des études n'ont pas déterminé nettement les règles rigoureuses d'application relatives à l'évaluation formative. La plupart du temps, ce type d'évaluation était considéré comme une activité d'autocorrection et respectueuse du cheminement de l'apprenant.

L'ensemble des recherches des trois tendances poursuivaient, malgré leurs différentes aspirations, un but commun: porter la maîtrise du français écrit à un plus haut niveau. D'un côté, on tente de dresser des portraits statistiques de la situation et, d'un autre, on tente des méthodes d'intervention didactiques et même formatives. Les études regroupées à l'intérieur de la première tendance, à prédominance statistique, ont montré qu'il y avait 
des difficultés réparties à plusieurs niveaux. Une grille était parfois utilisée afin de réaliser des portraits diagnostiques. Quant à elles, les études de la deuxième tendance cherchaient, pour leur part, à trouver une méthode d'intervention. Une grille était aussi parfois utilisée, mais cette fois-ci comme outil diagnostique d'intervention dans le but de guider la correction, puis d'intervenir sur les difficultés perçues en français. Finalement, les chercheurs de la troisième et dernière tendance ont montré l'utilité d'adopter des méthodes formatives et ce, surtout, pour le respect du cheminement d'apprentissage des élèves.

Les trois tendances ont montré à quelques endroits l'utilité d'utiliser une grille. C'est le projet que désire poursuivre la recherche présentée dans ces pages, soit d'entreprendre une démarche d'intervention de correction à l'aide d'une grille. Les études recensées amènent à croire qu'il faille continuer les recherches et qu'il faille cibler l'outil et/ou la méthode appropriée en regard des objectifs visés et ce, en vue d'atteindre un niveau acceptable en français écrit. Ceci amène à préciser l'orientation de la présente recherche, ainsi que son cadre spécifique.

\subsection{L'ORIENTATION ET LE CADRE DE LA RECHERCHE}

Les études recensées ont montré l'importance de corriger et ce, afin d'intervenir efficacement sur les difficultés, pour ensuite faire progresser les apprenants et leur permettre d'améliorer leur niveau de maîtrise en français écrit. Or, une correction articulée se doit de profiter d'outils qui permettent de cibler les difficultés afin d'intervenir efficacement. L'outil privilégié est une grille. L'utilisation d'une grille de cet ordre a été proposée dans les études de Beaulieu, Bourgeau et Paquin (1978), Jean, Leclerc et Racine (1988) et dans celle de Des Lauriers et Major (1992). La correction à l'aide d'une grille articulée permettrait au maître d'intervenir sur les faiblesses à saisir en français écrit et qui seront saisies 
dans le dialogue que l'utilisation de la grille va permettre d'entreprendre en correction. Or, un premier problème qui se pose est de savoir quel est le rôle et la composition d'une grille dans une correction de qualité. Il importe de fait, de donner les raisons d'utiliser une grille en correction. Il semble en effet que pour cibler les difficultés et faire une correction efficace, la grille en question doit présenter une taxinomie qui permette aux scripteurs de nettement situer leurs difficultés. Chacune des erreurs doit donc trouver sa classification. Le travail de recherche exigera en conséquence une analyse fine autant de l'ensemble des composantes de la grille qu'un examen approfondi de chacune des classes de fautes.

Un deuxième problème se pose, celui de l'utilisation de la grille. Une grille de correction doit pouvoir être réconciliée avec une approche qui tienne compte des apprentissages. Utilisée dans le quotidien sous forme d'outil didactique, la grille pourrait peut-être même rejoindre une vision non pas traditionnelle, mais plutôt une vision nouvelle en correction, celle d'une approche d'évaluation formative, rejoignant les applications tentées par Des Lauriers et Major (1992), Gagné (1986) et Roberge (1993). Si l'approche formative peut rejoindre de façon satisfaisante l'usage de la grille, il importera d'énoncer les principes permettant ce rapprochement et pouvant guider la correction. Ces orientations amènent à circonscrire avec précision et les objectifs de la recherche et le cadre qui leur est assigné.

\subsubsection{Les objectifs de la recherche}

C'est dans le cadre d'applications pédagogiques qu'ont surgi les objectifs présentés à l'intérieur de la figure 2. De plus, les essais exploratoires auprès d'une clientèle universitaire ont servi à alimenter la réflexion et à élaborer un matériel de soutien, complémentaire, mais fort important. 
1 Choisir et justifier les éléments d'une grille de correction des productions écrites a haute valeur diagnostique et qui mène le scripteur à réaliser des apprentissages.

2. Réconcilier la grille avec une approche nouvelle de correction formative et, par le fait même, déterminer quelques principes sous-jacents à l'utilisation d'une telle approche.

3. Élaborer, à titre de modeles, quelques exercices de soutien à la correction.

Figure 2: Élaboration des objectifs spécifiques de la recherche.

Par rapport au premier objectif, l'essentiel du travail de recherche en sera un de dépouillement et d'analyse ayant pour but l'élaboration d'une grille détaillée et sophistiquée de correction. Chacune des catégories seront proposées et décrites. Pour certaines, des exemples de classification seront montrés. De plus, un guide de correction intitulé: Guide de correction référentielle et interactive (voir annexe 1) a été conçu pour les correcteurs. Il contient plusieurs exemples-types de classification et des définitions des catégories et des sous-catégories utiles pour la correction.

En rapport au deuxième objectif, une réflexion sera faite concernant la correction; cette dernière ne pouvant être autrement que formative. Les théories de l'évaluation formative seront ainsi discutées, mais ce qui importe c'est que de larges principes formatifs de correction seront émis et ce, afin de guider nettement la correction et afin de guider l'apprentissage qui se veut formatif.

En rapport au troisième et dernier objectif, il se greffera un travail complémentaire d'élaboration de matériel didactique se voulant indicatif des voies s'offrant aux maîtres. Toutefois, une seule démonstration, pour quelques apprentissages seulement, sera faite et ce, uniquement dans le but d'illustrer. 
L'orientation que s'est donnée la recherche oblige à justifier ces choix. La prochaine et dernière partie de ce chapitre veut présenter le cadre d'étude dans lequel seront poursuivis les objectifs de ladite recherche.

\subsubsection{Le cadre de l'étude}

La présente recherche-développement, de portée qualitative, se tourne vers l'analyse conceptuelle pour encadrer son développement. Or, l'analyse conceptuelle, de nature théorique, est une méthode d'analyse peu répandue. Toutefois, ce mode d'analyse des données est celui qui s'applique précisément au type de recherche qui a été mené.

Pour Gagné et al. (1989, p.42), la démarche d'analyse conceptuelle présente «[...] des réflexions qui portent sur une définition de quelques concepts». L'analyse conceptuelle touche la connaissance. Elle a pour principale fonction ou de décrire, ou d'expliquer, ou d'élaborer des concepts théoriques, donc de nouvelles connaissances, de nouveaux savoirs. C'est de fait un raisonnement faisant référence à des concepts ou à des réalités que le chercheur tente d'expliquer et de justifier, soit à l'aide de théories, soit à l'aide de réflexions ou d'expériences menées sur le terrain. Dans le présent cas, les objets de connaissance seront ceux d'une grille comme outil (premier objectif) et d'une approche formative comme méthode (deuxième objectif). Il se greffera par la suite une présentation du matériel didactique qui se veut complémentaire et qui a pour but d'illustrer les possibilités d'application (troisième objectif).

Les travaux et les réflexions touchant le premier objectif feront partie du deuxième chapitre. Le troisième chapitre touchera les deuxième et troisième objectifs. Le lecteur attentif sera amené à constater que l'habituel «Cadre théorique» est partie intégrante de la production des connaissances de ces deux chapitres. 


\section{CHAPITRE II}

Les fondements et les catégories

d'une grille de correction 
Le premier problème de cette recherche touche au rôle et aux composantes d'une grille de correction. Il est rapidement apparu dans les essais exploratoires que la valeur et le rôle d'une grille de correction tenaient à sa composition même. C'est donc à une analyse fine et à l'établissement d'une grille de correction avec ses catégories et sous-catégories que la recherche s'est attaquée. Le résultat est exposé dans les pages de ce deuxième chapitre. L'exposé s'arrête toutefois d'abord à la présentation de la grille qui a servi de point de départ au travail.

\subsection{LES FONDEMENTS DE L'ANALYSE}

Le travail de recherche a pris comme point de départ la grille utilisée dans la recherche menée entre 1988 et 1991 auprès d'étudiants universitaires par les professeurs Boyer de l'Université du Québec à Hull, Roy de l'Université du Québec à Chicoutimi et Roy de l'Université du Québec à Rimouski (voir annexe 2). Leur étude avait pour but de «[...] mesurer l'impact de la formation sur la qualité du français écrit des futurs enseignants [inscrits en enseignement préscolaire-primaire à l'intérieur du réseau de l'Université du Québec] (Boyer, Roy, Roy, 1991, p.41)». Un article paru dans Québec français, intitulé: «Les enseignants apprennent-ils à écrire à l'Université?», faisait part des résultats de leur recherche et surtout des nouvelles catégories contenues dans leur grille qui avaient permis des analyses de fautes de haute qualité. Cette grille a été adoptée comme grille «A» de base. Grâce à la nouveauté et surtout à la justesse de certaines sous-catégories de cette grille, il a été possible de préciser des difficultés très fines dont celles relatives à la structure de la phrase et à l'usage des virgules. La grille utilisée à l'intérieur de la recherche de Boyer, Roy et Roy (1991) est apparue un choix valable pour, entre autres, la qualité des 
diagnostics qu'elle permettait et ce, grâce aux catégories et aux sous-catégories qu'elle contenait, ce que laisse voir la figure 3. C'était un choix des auteurs de cette grille «A» de retenir uniquement les catégories relatives à la forme.

\begin{tabular}{|ll|}
\hline 100 & Orthographe d'usage \\
200 & Morhhologie \\
300 & Accord \\
400 & Structure de la phrase \\
500 & Ponctuation et majuscule \\
600 & Lexique \\
\hline
\end{tabular}

Figure 3: Catégories de la grille «A» de base (Boyer, Roy et Roy, 1991).

La grille «B» finale de l'analyse a été construite à partir de cette grille «A». Plusieurs modifications ont été apportées, ainsi que des ajouts et des réorganisations. Les modifications apportées à la grille originelle sont issues principalement d'expérimentations faites au cours des sessions d'études universitaires comprises entre les sessions d'automne 1992 et d'hiver 1995. La grille était constamment utilisée pour la correction des productions écrites, principalement des dissertations et des comptes rendus, des étudiants en enseignement préscolaire-primaire de l'Université du Québec à Chicoutimi, inscrits aux cours de Didactique du français I et II. À la suite des expérimentations, des catégories se sont ajustées et c'est ce qui a conduit la grille à connaître 12 versions modifiées. La douzième version a pleinement satisfait les correcteurs. C'est donc sur cette grille finale - douzième version - que reposeront les explications.

Or, avant d'aborder le contenu de chacune des neuf catégories de la grille finale, il importe d'abord de présenter les fondements d'élaboration de la grille. La recherche a orienté ses choix vers une approche grammaticale ayant comme point d'appui la grammaire dite traditionnelle. De tous les types de grammaire, c'est celui de la grammaire traditionnelle qui convenait le mieux à la construction de la grille. 
Dans l'ouvrage intitulé La grammaire, Flaux (1993) identifie deux courants faisant référence à la grammaire traditionnelle: la grammaire d'usage ou normative et la grammaire raisonnée. Ces deux courants de la grammaire traditionnelle differrent quelque peu, quoique les deux reflètent bien les orientations grammaticales que s'est données la grille de correction. Flaux (1993, p.12) dit de la grammaire d'usage ou normative qu'elle «[...] s'intéresse aux points de la langue qui font difficultés [et qu'elle] ne peut négliger l'étude des règles générales». Cette définition rejoint les principes d'élaboration de la grille dont il est question. Car les difficultés à cibler chez les scripteurs, lors de la correction des productions écrites, réfèrent justement aux règles générales d'écriture et, donc, souvent à celles causant le plus de difficulté. Pour sa part, la grammaire raisonnée s'intéresse plus globalement aux phénomènes de la langue. Pour Flaux (1993, p.15), «héritière de la tradition latine, la grammaire raisonnée propose une description de la langue centrée sur les parties du discours (nom, verbe, pronom, adverbe, etc.)». Pour la finesse des diagnostics qu'il sera possible de réaliser à l'aide des sous-catégories de la grille, ce principe a été conservé par les concepteurs de la grille et ce, pour les catégories s'y prêtant le mieux: 200, de Morphologie et 300, d'Accord.

Choisir la grammaire traditionnelle afin de déterminer les catégories et les sous-catégories de la grille plutôt qu'un autre type de grammaire est dû aussi, et surtout, à des raisons utilitaires. Il fallait que la grille soit pratique et facile d'utilisation. Étant donné que ce seront des enseignants qui utiliseront cet outil de correction, il importait de présenter des catégories et des sous-catégories connues et correspondant à ce qu'utilisent les maîtres en salle de classe. Pour les élèves aussi, car si la grille veut leur indiquer clairement les fautes, et par le fait même leur faire comprendre leurs erreurs, les catégories et les sous-catégories devaient correspondre à ce que les élèves connaissent déjà. Ainsi, la grille se devait de respecter ce qui se pratique dans les classes, donc ce qui est connu. 
Toutefois, la grille ne pouvait se construire à l'aide de la seule grammaire. Il fallait faire appel à un autre outil d'élaboration, qu'est la «science» de la pratique, soit la pragmatique. À vrai dire, la pragmatique n'est pas une science, mais plutôt une intuition qui se développe par la pratique. La pragmatique dans le sens de «la pratique» qu'elle se donne peut faire l'objet de rapprochements avec la didaxologie. La didaxologie se définit comme étant une «méthodologie générale basée sur la recherche empirique (De Corte et al., 1979)». Dès lors, la pragmatique s'efforce de trouver, par sa recherche empirique, les éléments ou les notions qui émergent de l'action didactique, donc de la pratique. De ce fait, la pragmatique, qui se donne pour objet de considérer la valeur pratique des choses, est aussi au coeur de l'organisation profonde de la grille. Au cours des expériences de correction, les concepteurs de la grille ont constamment tenu compte des éléments externes d'expérimentation afin d'ajuster ou de modifier certaines catégories ou sous-catégories de la grille. Les catégories ayant davantage profité de la pragmatique sont la catégorie 400 Structure de la phrase et les trois dernières catégories $(700,800$ et 900$)$ touchant le fond du texte.

Les choix théoriques d'élaboration de la grille ont été, d'une part, la grammaire traditionnelle: elle avait pour tâche d'apporter les éléments de la langue à respecter en écriture et, d'autre part, la pragmatique: elle avait pour tâche d'apporter et d'ajuster les notions de la pratique réelle de correction de la production écrite. Ces deux choix semblent être une combinaison judicieuse et ce, en raison de leur complémentarité.

On pourrait ne pas être en accord avec ces choix. Idéalement, il aurait été souhaitable d'élaborer, par exemple, toutes les théories relatives à la linguistique, à la morphologie, à la syntaxe, à la sémiologie et même à la rhétorique, entre autres, mais cela apparaît quelque peu utopique dans le cadre que s'est donné la recherche. L'entier des champs 
couverts par la langue française sont retenus dans la grille et sont trop larges pour en faire une étude approfondie. D'ailleurs, là n'est pas le but de la recherche; il vaut mieux, ici, présenter et justifier les choix de pareille grille. La figure 4 fait voir l'ensemble des catégories de la grille «B». Il a été retenu, pour cette grille finale, les six catégories de la grille «A» auxquelles il a été ajouté trois autres catégories.

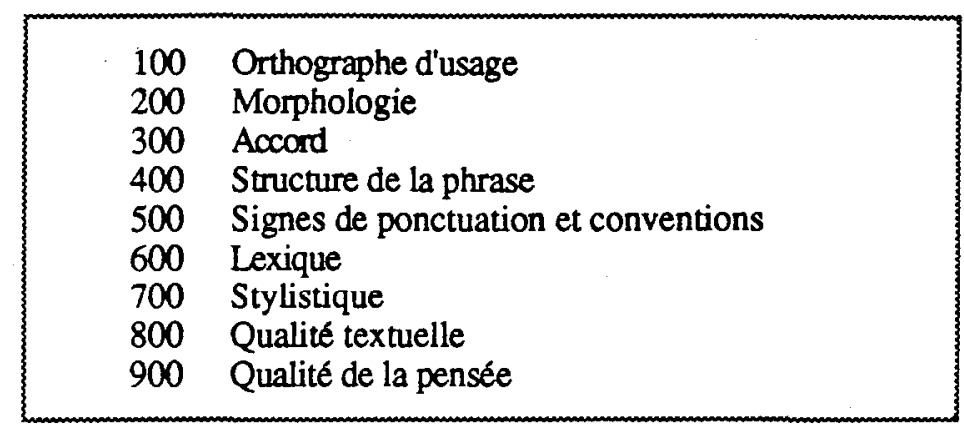

Figure 4: Catégories de la grille «B» finale (voir annexe 3).

\subsection{LA GRILLE DE CORRECTION}

L'analyse qui sera faite de la grille de correction va débuter par la présentation des six premières catégories, par la suite les explications se poursuivront pour les catégories additionnelles touchant plus spécifiquement le fond d'un texte. Suit la présentation des sous-classes de ces catégories.

\subsubsection{La catégorie 100: Orthographe d'usage}

La grille s'ouvre par la catégorie d'Orthographe d'usage. Traditionnellement, «l'orthographe d'usage a pour objet les mots pris en eux-mêmes, tels que les donne le dictionnaire, sans égard à leur rôle dans le discours (Grevisse, 1980, p.147)». Pour Goosse (1986, p.100), la définition est la même, sauf qu'il modifie légèrement la donnée finale 
qui devient fort justement «[...] sans égard à leur rôle dans la phrase». La première définition, celle de Grevisse, donne à penser, ainsi que le relève l'auteur lui-même (Grevisse, 1980, p.147), que l'apprentissage de l'orthographe des mots est avant tout d'observation et de mémoire. Grevisse se corrige toutefois en référant à l'utilité d'une certaine connaissance de l'étymologie. Il dira même que «l'orthographe actuelle est dite étymologique (Grevisse, 1980, p.147)» et dans l'analyse qu'il en fera, il s'arrêtera à souligner les insuffisances de l'orthographe française.

Dans sa version refondue du Bon usage de Grevisse, Goosse (1986) est autrement plus constructif et l'histoire qu'il fait de l'orthographe française fait mieux ressortir le cheminement d'un système qui se cherche. Référant à Thimonnier, il écrit que Thimonnier est «convaincu que notre orthographe est moins absurde, moins anarchique qu'il n'est tradition de le dire et qu'elle forme un système assez cohérent pour se prêter à une analyse raisonnée (Goosse, 1986, p.102)».

Dans ce cadre conceptuel d'analyse raisonnée de l'orthographe d'usage, la catégorie «Orthographe d'usage» d'une grille de correction retiendra des erreurs d'une nature à clairement définir. Or, Grevisse définit l'objet de cette catégorie comme «les mots pris en eux-mêmes». L'expression reste quelque peu vague. Ainsi, l'idée proposée par ce grammairien «d'utiliser comme critère d'identification de ce type de faute le fait que la forme correcte puisse se retrouver au dictionnaire» peut se révéler dans le quotidien très pratique. La nuance à introduire pour marquer une séparation plus nette de cette catégorie pourrait être celle de la distinction sémantique et grammaticale. Au total donc appartiendraient à la catégorie Orthographe d'usage la ou les parties du mot qui ne présentent pas de dimensions grammaticales. Tous ces propos, si intéressants qu'ils soient, présentent toutefois comme faiblesse de ne pas justifier de cadre à la catégorie Orthographe d'usage. 
L'orthographe est à la fois historique, étymologique et sémantique. Que l'orthographe soit historique s'explique par l'origine de graphies puisées à d'autres langues. Car les mots se construisent au cours des siècles et laissent sur leur passage certaines données du temps passé. Tel est le cas, par exemple, pour la graphie «oi» contenu à l'intérieur du mot «roi». Logiquement ce mot se prononcerait [roy]; mais le mot en est venu à se prononcer [rwa], passant de [regem] en latin, à [rei], [roi] et finalement à [rwa].

Par ailleurs l'orthographe peut être étymologique et ce, en référence aux emprunts de langue. Le français actuel est en grande proportion constitué du latin. Or, notre langue a emprunté plusieurs mots à d'autres langues, emprunt que laisse souvent percevoir la graphie française. Par exemple, la graphie «qu» peut se révéler un emprunt à une langue amérindienne; la graphie «ph» renvoie, pour sa part, au grec. Même que certains mots complets ont été puisés à d'autres langues: sandwich, pouding, fjord, match par exemple, ce qui justifie l'orthographe particulière de ces mots.

Finalement, l'orthographe est sémantique en ce qu'elle réfere au sens des mots. La justification de cette orthographe sémantique s'explique notamment par l'existence d'une parenté orthographique. À titre d'exemple, il importait de réintroduire le «g» et le «t»à l'intérieur du mot «doigt», ce dernier étant intimement lié à un mot de même famille, comme «digital». Tel sera aussi le cas pour le mot «vingt» ne pouvant faire abstraction du mot de même famille «vingtaine».

Les exemples de ce type sont légion. À ce propos Thimonnier (1974), dans son Code orthographique et grammatical, montre bien toute l'évolution historique de l'orthographe. Même dans le cas où Thimonnier s'interroge sur certaines orthographes particu- 
lières, le lecteur ne peut s'empêcher de penser que certains emplois en apparence aberrants pourront eux aussi trouver leur explication dans un système explicatif prolongé.

Il y aurait donc une grammaire de l'orthographe et il est acquis que, dans les écoles, et pour l'essentiel, cette grammaire n'est pas présentée. Les maîtres ne l'enseignent qu'occasionnellement; ils n'ont pas les outils pour le faire, car les recherches sur le sujet n'ont pas été menées à termes. Une grammaire de l'orthographe est donc à faire et les besoins sont énormes. L'idéal serait que des notions orthographiques plus précises soient enseignées aux apprenants. Car l'écriture ne fait pas qu'écrire les mots d'orthographe: elle exige une représentation juste de ses systèmes et élabore l'origine et la logique, parfois oubliées, de plusieurs mots d'orthographe d'usage. C'est pour ces raisons que nombre de propositions, faites à l'intérieur de différents essais de réforme orthographique, ont rencontré des oppositions, car elles vont contre le système. C'est que ces propositions viennent contredire la conviction de plusieurs spécialistes et non-spécialistes que les propositions en question sont inacceptables dans le cadre des règles, intuitivement perçues, qui gouvernent l'écriture.

Ainsi il faudrait, dans l'apprentissage de l'orthographe d'usage à l'école, tenir compte des phénomènes de la formation des mots et ce, au lieu de condamner les élèves à des listes de mots à apprendre par coeur. Il faudrait rendre compte de la logique de certains mots d'orthographe, car les différentes graphies des mots répondent à des impératifs ou de parenté orthographique, ou d'étymologie, ou de sens logique.

De ce fait même, les sous-catégories de la catégorie Orthographe d'usage de la grille devraient aussi rendre compte des notions précises de l'orthographe. Or, les recherches peu avancées sur le sujet amènent à poser qu'il devient impossible, étant donné le cadre de 
la grammaire traditionnelle que s'est donné la grille, d'ériger des sous-catégories se rapportant par exemple à l'histoire, à l'étymologie ou au sens des mots d'orthographe d'usage. Seuls des spécialistes de la langue peuvent cibler avec exactitude l'origine des mots et donner les explications possibles.

La catégorie Orthographe d'usage de la grille se limite donc à trois sous-catégories qui semblent faire judicieusement état des difficultés les plus nettes et les plus fréquentes en écriture des mots d'orthographe d'usage, ce que laisse voir la figure 5. La première sous-catégorie Cas général englobera tous les cas d'orthographe d'usage, tels que définis précédemment, autres que ceux se retrouvant aux deux sous-catégories restantes: Signes auxiliaires et Géminée.

$$
\begin{array}{ll}
110- & \text { Cas général } \\
120- & \text { Signes auxiliaires } \\
130 & \text { Géminée }
\end{array}
$$

Figure 5: Sous-catégories de la catégorie 100: Orthographe d'usage.

La deuxième sous-catégorie Signes auxiliaires relève les fautes d'accents (circonflexe, aigu, grave), du tréma, de la cédille, de la séparation des mots, de l'apostrophe et du trait d'union. Une modification pour cette sous-catégorie fut introduite comparativement à la grille «A» de base. Au départ, cette sous-catégorie appelée Accents ne contenait que les cas d'accents. Cette sous-catégorie fut ainsi mieux précisée, c'est-à-dire que tous les autres signes ont été introduits. L'appellation attribuée à la sous-catégorie Signes auxiliaires a fortement été inspirée de celle de Grevisse (1980, p.58), «Signes orthographiques». 
Finalement, la troisième sous-catégorie Géminée regroupera toutes les fautes faisant référence aux règles de la géminée. «Il y a géminée lorsqu'il y a succession de deux consonnes identiques (Goosse, 1986, p.45).» Ce sera ainsi le cas d'une géminée ou omise (aporte), ou introduite à tort (remmettre). Ordinairement, les cas de géminée inventée (proffesseur) seraient à classer à l'intérieur de la sous-catégorie 110- Cas général. Or, pour les besoins du maître et pour ceux de ses enseignements, il est conseillé de regrouper ensemble tous les cas d'emploi de la géminée, soit fautifs, soit absents.

Un maitre qui voudra indiquer à son scripteur ses difficultés orthographiques pourra le faire à l'aide de ces trois sous-catégories. Le maître pourra aussi transmettre un portrait diagnostique valable qui permettra de fournir des informations pertinentes par rapport aux difficultés perçues. Or pour l'instant, tout un matériel serait à construire pour cette catégorie, mais d'abord des recherches sont à mener. Il est à souhaiter qu'un jour les maîtres puissent enseigner l'orthographe d'usage à leurs élèves et que ces derniers puissent aussi la comprendre. Car les graphies de plusieurs mots d'orthographe ont un sens propre et, parfois même, une logique.

Pour une autre organisation en sous-catégories de la catégorie Orthographe d'usage, il appartiendra au maître de déterminer quelles erreurs il veut particulièrement diagnostiquer. C'est ainsi que le maître ayant enseigné à propos de l'orthographe des nasales l'usage de l'm devant le «p» et le «b» pourrait décider d'ériger en sous-catégories ces orthographes. Des cas comme celui-ci sont nombreux. Le choix qui a été fait, ici, de diviser cette catégorie en trois sous-catégories peut s'ajuster et ce, selon les besoins des maîtres et des classes. Pour plus de détail, le lecteur est invité à consulter le Guide de correction référentielle et interactive (voir annexe 1); il contient plusieurs exemples-types et des explications 
des trois sous-catégories retenues pour la catégorie Orthographe d'usage, de même que pour toutes les autres catégories et sous-catégories contenues à l'intérieur de la grille.

La prochaine catégorie Morphologie est intimement liée à la catégorie Orthographe d'usage. Ces deux catégories initiales de la grille entretiennent des rapports étroits malgré leur apparente différenciation. Connaître la logique de certains mots d'orthographe d'usage, c'est en connaître le système. Les différentes natures de mots possèdent un système, donc des variations. Le mot en catégorie Orthographe d'usage est vu isolément, comme le donne le dictionnaire et sans variations grammaticales, mais le mot en catégorie Morphologie s'attache, pour sa part, à un système donc à des variations. Ce sera ainsi au maître de faire un choix devant certains cas, soit d'orthographe d'usage, soit de morphologie. Il devra ainsi juger s'il y a système et si celui-ci doit être maîtrisé, ou plutôt si les variations en système doivent être connues par le scripteur. Des explications et des exemples de la deuxième catégorie: Morphologie sont nécessaires.

\subsubsection{La catégorie 200: Morphologie}

Traditionnellement, dans une grille ne contenant pas de catégorie Morphologie, la faute de morphologie est versée à la catégorie des erreurs d'orthographe d'accord, ou même d'orthographe d'usage. La nuance à faire jouer peut paraître à première vue ténue. Toutefois, l'erreur de morphologie se veut celle qui montre la méconnaissance d'une donnée préalable à l'exercice de la syntaxe d'accord.

La catégorie Morphologie réunit toutes les fautes qui relèvent des variations de formes que subit un mot eu égard aux paradigmes grammaticaux et même orthographiques. Il importe de distinguer la faute de morphologie, ce type de faute résultant plutôt de la mé- 
connaissance de l'orthographe d'une forme selon sa nature de mot. Connaître la morphologie d'un mot, c'est connaître les transformations grammaticales possibles du mot et choisir la graphie qui convient pour un emploi donné. À titre d'exemple, la faute je les ai envoyer est à placer parmi les fautes de morphologie du verbe, le scripteur ne distinguant pas l'infinitif du participe passé. Au total les erreurs de morphologie sont celles qui touchent aux formes que commandera l'accord, mais uniquement en regard de ce que doivent être ces formes dans le cadre du paradigme auquel les erreurs appartiennent.

C'était un choix de la grille de départ de reconnaître une catégorie Morphologie. La distinction d'une catégorie de ce type, où il soit possible de rassembler des erreurs caractéristiques de morphologie, ne se présente que dans peu d'études ou de recherches. Cette catégorie se retrouve aussi dans bien peu de grilles qui classent erronément les erreurs soit dans la catégorie Orthographe d'usage, soit dans la catégorie Accord privant ainsi et le maître et l'élève d'informations précieuses sur la nature des erreurs qui auront été faites. À la limite, les éudes ayant inclus une catégorie Morphologie à leur grille faisaient référence tout au plus à des notations partielles de difficultés de morphologie, celle de l'homophonie par exemple, une mode récente des programmes officiels et de plusieurs grammairiens. Car nombre de ces derniers présentent une ou des pages de grammaire appelées: Homophones, considérant ainsi cette catégorie comme une règle de grammaire. La plupart des fautes présentant des caractéristiques d'homophonie appartiendront, donc, à la catégorie Morphologie. Or, la présente grille identifiera la difficulté homophonique en référant le scripteur à la nature même du mot qu'il a employé. Ainsi, il sera indiqué au scripteur ayant écrit mais amis, une faute de morphologie: 230 - Article et adjectif déterminatif (possessif) et non pas une faute d'homophones qui de fait, n'oriente nullement le scripteur vers la compréhension de sa faute. 
La catégorie Morphologie retiendra donc comme sous-catégories les différentes parties du discours comme le suggère la grammaire dite traditionnelle. Les sous-catégories sont présentées à l'intérieur de la figure 6. Une modification fut introduite pour cette catégorie comparativement à la grille «A». Au départ, la sous-catégorie 220 prenait comme appellation Adjectif, regroupant autant les adjectifs qualificatifs que les adjectifs déterminatifs. Il a ainsi été convenu pour la sous-catégorie 220 de la préciser mieux et ce, pour la qualité des diagnostics qui seront transmis aux scripteurs. De ce fait, la sous-catégorie 220 ne regroupe maintenant que les cas d'adjectifs qualificatifs et la sous-catégorie 230 que les cas d'articles et d'adjectifs déterminatifs.

Les propos qui suivront, ainsi que les quelques exemples de classification, aideront à mieux saisir l'objet et la nature de cette catégorie Morphologie plutôt nouvelle en correction. Car, distinguer une catégorie Morphologie, et donc un ensemble d'erreurs pouvant être qualifiées d'erreurs de morphologie, demande une réflexion fine sur la nature de cette sorte d'erreur.

$$
\begin{array}{ll}
210- & \text { Nom } \\
220- & \text { Adjectif qualificatif } \\
230- & \text { Article et adjectif déterminatif } \\
240- & \text { Pronom } \\
250- & \text { Verbe } \\
260- & \text { Adverbe } \\
270- & \text { Préposition } \\
280- & \text { Conjonction de subordination } \\
290- & \text { Conjonction de coordination } \\
295- & \text { Interjection }
\end{array}
$$

Figure 6: Sous-catégories de la catégorie 200: Morphologie.

Dans le cadre d'un projet-milieu de deuxième cycle universitaire en Éducation, une recherche a été menée auprès d'une classe de cinquième année du primaire par Jean (1992). L'étude, ayant pour titre: Projet de recherche sur la maîtrise d'écriture des formes 
verbales par des enfants de cinquième année du primaire, poursuivait comme objectif de mesurer et d'augmenter la maîtrise de l'écriture, mais aussi d'identifier et de préciser la nature des difficultés se rapportant plus spécifiquement au verbe. La grille de classification utilisée comprenait justement une catégorie Morphologie. Elle a permis de montrer que les erreurs commises par les élèves étaient à $66 \%$ des erreurs de morphologie du verbe, plutôt que des erreurs d'accord, comme le croyait et le voyait au départ l'enseignante qui avait demandé l'intervention diagnostique. Un total de 268 fautes sur 404 ont été classées en catégorie Morphologie du verbe. Ce verdict avait permis à l'enseignante de prendre conscience qu'il manquait tout un créneau à son enseignement, créneau qui était apparu à l'examen négligé d'ailleurs même par le programme. Ce verdict remettait aussi en considération toute l'ignorance de certains paradigmes verbaux non maîtrisés par cette clientèle de cinquième année du primaire.

Tel est aussi le cas pour le secondaire et pour les niveaux plus élevés lorsque les apprentissages ont retardé indûment où n'ont pas été faits au moment où ils auraient dû se faire, par exemple ceux du verbe. C'est ainsi qu'il sera possible de retrouver beaucoup d'erreurs de morphologie dans les copies de scripteurs qui ont une maîtrise incomplète de leur langue écrite. Par exemple les erreurs, contenues à l'intérieur des figures 7,8 et 9 correspondent à ce qui se définit comme des fautes de morphologie. Suivent les explications; d'abord celles se rapportant à la sous-catégorie Morphologie du nom.

$$
\begin{aligned}
& \text { 1- Une veufe } \\
& \text { 2- Des pneux } \\
& \text { 3-Une avion }
\end{aligned}
$$

Figure 7: Exemples d'erreurs à classer à l'intérieur de la sous-catégorie 210: Morphologie du nom. 
Lorsque le scripteur commet une erreur de morphologie à l'intérieur des noms (210), c'est qu'il ne connaît pas réellement les variations possibles résultant du genre et du nombre des noms. Tel sera le cas pour les deux premiers exemples Une veufe (1) et Des pneux (2). Dans un cas, le scripteur ne maîtrise pas les règles de transformation du masculin au féminin de ce mot. Dans l'autre cas, il est net que le scripteur ne connaît pas les variations en nombre de l'accord au pluriel de ce mot.

Le troisième exemple une avion apparaît aussi devoir être classé à la sous-catégorie Morphologie du nom et non pas à la sous-catégorie Accord du déterminant (330). L'erreur origine en effet plutôt de la méconnaissance d'une caractéristique première du nom, qu'est son genre. Voir ici une faute d'accord ne permet pas de situer avec exactitude la nature de la difficulté et les apprentissages que devra faire le scripteur.

Pour leur part, les exemples (1) et (2) de la figure 8 correspondent à l'erreur type de morphologie des adjectifs qualificatifs. Toutefois, le correcteur pourra encore une fois choisir de classer la faute Une table légerte en catégorie 640- Barbarisme et ce, étant donné la construction inventée de ce mot. Le choix appartiendra ainsi au maître et ce, dépendamment de la notion qu'il voudra faire acquérir. Car si ce cas est considéré comme étant une erreur de morphologie des adjectifs qualificatifs, il sera plus aisé de faire intervenir la logique de l'erreur et de corriger sa mauvaise transformation en genre.

1-Une table légerte

2- Un test diagnostic

Figure 8: Exemples d'erreurs à classer à l'intérieur de la sous-catégorie 220: Morphologie des adjectifs qualificatifs.

Or, l'exemple Un test diagnostiç (2), est discutable. Un correcteur pourra ainsi décider de classer ce cas en catégorie Orthographe d'usage. Or, étant donné que le scripteur 
orthographie ce mot selon une nature de nom et qu'ici il est employé comme adjectif, il est alors jugé qu'il ne connaît pas les transformations de ce mot employé selon sa nature idjectivale.

L'exemple de faute Vingts amis (1) de la figure 9 présente une erreur de morphologie des adjectifs numéraux (230). Le scripteur n'a pas retenu que les nombres ne prennent pas de -s dans ce cas, ou bien ne sait pas que la valeur sémantique ne s'accommode pas, pour ces numéraux, de la marque externe de $-s$ du pluriel. Un nouvel enseignement sera ou nécessaire ou à reprendre et ce, pour corriger cette difficulté ou pour faire comprendre le principe de la pluralité interne des nombres.

$1-$ Vingts amis
$2-11$ s'occupe de ces affaires

Figure 9: Exemples d'erreurs à classer à l'intérieur de la sous-catégorie 230: Morphologie des articles et des adjectifs déterminatifs.

Pour sa part, l'exemple Il s'occupe de ces affaires (2) montre une non-maîtrise des adjectifs démonstratifs et possessifs. Certains concepteurs de grille érigent une catégorie «Homophones» pour ce type d'erreur. Or, pour la faiblesse des diagnostics transmis et pour l'impossibilité d'en tirer un enseignement logique et satisfaisant, il est davantage utile et pédagogique d'informer le scripteur sur les natures de ses confusions d'emplois.

L'exemple du type (1) présenté à la figure 10 sera aussi à classer à l'intérieur de la sous-catégorie 240-Morphologie du pronom, car le scripteur n'a pas reconnu un pronom dans cet emploi. De même, l'exemple (2) Ils sont bien gentils devra être classé à l'intérieur de la sous-catégorie 260- Morphologie de l'adverbe, car il semble que le scripteur, cette fois-ci, a orthographié le mot «bien» comme étant un adjectif. Il sera ainsi nécessaire 
de lui rappeler l'invariabilité de l'adverbe et de reprendre avec lui l'enseignement et l'apprentissage des caractéristiques de ce mot invariable qu'est l'adverbe.

$1-$ Ont les respecte
$2-$ Ils sont biens gentils
$3-$ Il veut mangé

Figure 10: Exemples d'erreurs à classer à l'intérieur des sous-catégories 240: Morphologie du pronom, 260: Morphologie de l'adverbe et 250: Morphologie du verbe.

Finalement l'exemple (3) de morphologie du verbe (250) Il veut mangé montre une méconnaissance, sinon une confusion des formes du participe passé et de l'infinitif. Ceci apparaît être une erreur de recenser cette faute comme une faute d'accord. L'erreur commise montre plutôt un manque de maîtrise d'un paradigme verbal élémentaire: voir la faute comme une faute d'accord, c'est introduire une distorsion diagnostique nuisible pour le maître qui situera moins bien les difficultés de l'élève et pour l'élève qui comprendra plus difficilement la nature spécifique de ses difficultés.

À l'expérience, le correcteur et le maître verront tout l'intérêt qu'il y a à rassembler les erreurs touchant à la Morphologie. Le maître ne pourra pas faire autrement que de se féliciter de la qualité du diagnostic obtenu et de la situation dans laquelle il se trouvera placé pour organiser un enseignement correctif.

L'exclusion par les programmes officiels de l'ensemble de la grammaire ces dernières années a fait perdre de vue l'importance qu'il y a à faire maîtriser les données de la morphologie. Or, un diagnostic établi à l'aide d'une grille de correction comprenant une catégorie Morphologie ne manquera pas d'indiquer très nettement qu'un très grand nombre des erreurs relevées dans les copies des étudiants sont des erreurs de morphologie. Ce 
qui est forcément le cas pour le primaire où peut-être plus qu'ailleurs ces notions doivent être enseignées.

Ce ne signifie pas qu'il faille mettre au rancart la catégorie Accord. Tout au contraire, une catégorie Accord est primordiale à l'intérieur d'une grille de correction. Or il a semblé, dans plusieurs autres grilles, que cette catégorie ait été négligée, voire même considérée quelque peu comme une catégorie «fourre tout». Ce que doit contenir une catégorie Accord doit nécessairement être des cas d'accord. Éliminant ainsi les cas relevant de la morphologie, la catégorie Accord s'en trouve alors mieux précisée dans ses emplois.

\subsubsection{La catégorie 300: Accord}

La troisième catégorie, la catégorie d'Accord, est le résultat de manifestations sémiologiques, donc de formes qui vont permettre l'accord. Pour Goosse (1986, p.686) «l'accord est le fait qu'un mot variable (receveur) reçoit d'un autre mot de la phrase (donneur) ses particularités morphologiques: son genre, son nombre et sa personne». De plus, selon leur nature, les mots répondent entre eux à des règles régies par l'accord des mots d'une phrase. À ce propos, Grevisse (1980, p.27) disait que «les règles d'accord sont fondées sur la logique et découlent nécessairement des relations créées par l'esprit entre les choses».

La catégorie Accord de cette grille regroupe donc les fautes qui résultent d'un choix d'une forme ne marquant pas adéquatement la correspondance entre deux mots dont l'un est subordonné à l'autre. Les sous-catégories retenues, les parties du discours, sont présentées à l'intérieur de la figure 11. La sous-catégorie Verbe est redivisée en plusieurs 
sous-sous-catégories et ce, pour l'ampleur des règles rattachées au verbe et aussi pour l'importance attribuée à la maîtrise de ces règles.

\begin{tabular}{|c|c|c|}
\hline $\begin{array}{l}310- \\
320- \\
330- \\
340- \\
350-\end{array}$ & $\begin{array}{l}\text { Nom } \\
\text { Adjectif } \\
\text { Article } \\
\text { Pronom } \\
\text { VERBE } \\
351- \\
352- \\
353- \\
354- \\
355- \\
356- \\
357- \\
359- \\
\text { Adverte }\end{array}$ & $\begin{array}{l}\text { ualificatif } \\
\text { adjectif déterminatif } \\
\text { Règle générale } \\
\text { Collectif sujet } \\
\text { Plusieurs sujets } \\
\text { Participe passé (seul) } \\
\text { Participe passé (être) } \\
\text { Participe passé (avoir) } \\
\text { Participe passé (pronominal) } \\
\text { Autres }\end{array}$ \\
\hline
\end{tabular}

Figure 11: Sous-catégories et sous-sous-catégories de la catégorie 300: Accord.

Les sous-catégories de cette catégorie ne semblent pas causer de problème. Tout correcteur initié sait reconnaître une faute d'accord. Toutefois, une difficulté qui peut se poser en correction est celle relative à la sous-catégorie 354 Accord des participes passés employés seuls. Cette sous-catégorie présente une difficulté de discrimination, celle de la nature d'adjectif ou de participe passé, illustrant un des principes majeurs d'élaboration et d'utilisation de la grille qui veut que le contenu même de la grille soit fonction et des connaissances grammaticales du maître et du niveau des apprentissages. C'est ainsi que pour certains, dans certains cas, au niveau le plus élémentaire, la nuance ne sera pas faite entre l'adjectif et le participe passé. À un niveau plus avancé, la nuance pourra être faite à partir des difficultés contextuelles que sont essentiellement les compléments accompagnant un mot ayant nature de verbe. Ce n'est qu'au niveau le plus avancé que la distinction fine du participe passé et de l'adjectif pourra être faite, et encore, sur la base de la portée sémantique des deux natures parentes d'adjectif et de participe passé. Il faut avouer, au total, que la question est fort difficile et que les explications restent partielles autant dans les meil- 
leurs traités de grammaire que dans les ouvrages de linguistique. Or, dans les deux cas, la règle sera la même: les adjectifs et les participes passés employés sans auxiliaire s'accordent en genre et en nombre avec le(s) nom(s) au(x)quel(s) ils se rapportent.

De plus, il est possible que le correcteur soit tenté de considérer une faute comme J'entend comme une faute d'accord. Toutefois, cette faute devrait être vue, pour les variations morphologiques de personne qu'elle présente, comme une faute de morphologie du verbe. Toutefois, à un niveau plus élémentaire, au primaire par exemple, où ces cas font l'objet d'apprentissages systématiques, le maître pourra choisir de considérer cette faute comme étant d'accord. Plus tard, lorsque ces règles seront acquises, et qu'elles ne feront plus l'objet d'un apprentissage, elles devront être considérées de catégorie Morphologie.

Au départ, la catégorie Accord de la grille «A» considérait comme sous-catégories additionnelles les autres parties du discours, c'est-à-dire les sous-catégories: Adverbe, Préposition, Conjonction de subordination, Conjonction de coordination et Interjection. Or, ces dernières ont été supprimées en raison de leur invariabilité, donc de leur impossibilité de s'accorder. Les erreurs réalisées pour ces mots invariables seront logiquement référées à la catégorie Morphologie étant donné que par définition un mot invariable n'accepte pas la variabilité d'accord et que le scripteur n'a pas retenu que ces mots possèdent un système d'invariabilité. Une seule sous-catégorie pour ces mots invariables a été retenue: Adverbe et encore pour le seul adverbe «tout» et pour les cas où la grammaire lui accorde —à tort d'ailleurs - la variabilité (exemple: toute prête).

La partie portant sur la catégorie Accord ne nécessite pas plus de développements, étant donné le caractère plutôt connu de cette catégorie. Ainsi, les erreurs à classer à l'intérieur de la catégorie Morphologie répondent aux variations d'un système, tandis que les erreurs à porter à la catégorie Accord répondent à des règles précises dont l'application ré- 
sulte des rapports qu'entretiennent les mots variables d'une phrase. Par contre, les mots pris dans un ensemble et répondant à certaines règles, mais cette fois-ci de structure, seront maintenant à considérer en catégorie Structure de la phrase.

\subsubsection{La catégorie 400: Structure de la phrase}

La catégorie Structure de la phrase est à son tour intimement liée aux catégories de morphologie et d'accord. Toutefois, même si elle présente certaines correspondances avec ces catégories, elle possède malgré tout ses particularités qu'il importe de distinguer; elle regroupe conséquemment les erreurs relatives à ces particularités.m Ainsi, la catégorie Structure de la phrase relève toutes les fautes qui touchent à l'organisation de la phrase, exceptions faites des fautes de morphologie et d'accord déjà inventoriées. Ici encore, il importe de bien distinguer les erreurs d'accord des erreurs de structure de phrase.

D'un point de vue théorique, la catégorie Structure de la phrase tend à être la catégorie où il est le plus difficile d'établir le cadre et d'identifier les limites. Or, en correction, cette catégorie regroupe des sous-classes d'erreurs très fréquentes qui se révèlent indispensables à l'intérieur d'une grille. Quelque peu «idiosynchratique», cette catégorie s'est construite et s'est ajustée au cours des expériences menées en correction et est donc issue autant de la pragmatique que de l'analyse théorique. Pour l'essentiel, les sous-catégories retenues avaient été proposées dans le projet de recherche Boyer, Roy et Roy (1991), une analyse pratique ayant permis d'établir une classification à partir des inventaires de fautes. Pour cette catégorie, les parties du discours ont été abandonnées. Les erreurs étaient ainsi regroupées selon leur parenté et leur fréquence. La classification proposée, telle que présentée à l'intérieur de la figure 12, est apparue dans les expérimentations de nature à bien situer les fautes. 


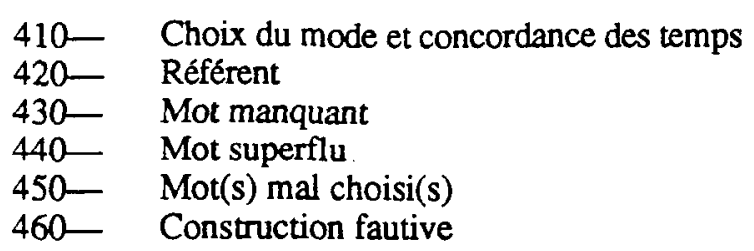

Figure 12: Sous-catégories de la catégorie 400: Structure de la phrase.

Les sous-catégories ont été identifiées et se présentent dans un ordre relevant du nombre des fautes commises par les étudiants auprès de qui l'étude avait été faite. La catégorie s'ouvre par une sous-catégorie Choix du mode et concordance des temps. Par la suite, en ordre et proportionnellement, les fautes commises étaient d'abord des fautes de référent, ensuite des fautes de mot manquant, de mot superflu et finalement de mot(s) mal choisi(s). Une sous-catégorie Construction fautive a aussi été ajoutée à l'ensemble de la grille originelle.

Étant donné les particularités de cette répartition, la catégorie Structure de la phrase se comprendra mieux par le biais des explications des cas possibles pour chacune des six sous-catégories. Des exemples sont présentés pour chacune des sous-catégories. D'abord la sous-catégorie Choix du mode et concordance des temps; les exemples de fautes apparaissent à la figure 13 .

1-Si j'aurais le temps
2-J'aimerais qu'il peut venir

Figure 13: Exemples d'erreurs à classer à l'intérieur de la catégorie 410: Choix du mode et concordance des temps.

Les erreurs classées à l'intérieur de la première sous-catégorie de la catégorie Structure de la phrase: Choix du mode et concordance des temps (exemples 1 et 2 ) sont consi- 
dérées à tort, dans beaucoup de grilles, comme des fautes d'accord. Malgré qu'il y ait un lien indiscutable avec la catégorie Accord, il importe de bien voir que les erreurs de choix de mode ou de concordance des temps résultent d'une analyse fautive de rapports grammaticaux et logiques qui appartiennent à l'entier de la phrase, comme le montrent d'ailleurs les deux premiers exemples. Ces erreurs ne sont donc pas des fautes d'accord, mais plutôt un mauvais choix de mode ou de temps dans l'organisation de la phrase. L'erreur réalisée dépendra avant tout de la rencontre des structures grammaticales choisies et non pas de l'accord de deux paradigmes. Le choix d'ouvrir la catégorie par des erreurs relatives à la structure de la phrase ne peut être mieux fait que par cette catégorie (Choix du mode et concordance des temps) montrant des relents d'erreurs d'accord.

Les exemples (1) et (2) de la figure 14 sont des fautes de référent, sous-catégorie 420. Ces fautes sont occasionnées par un choix fautif de mot eu égard au rapport que le mot entretient avec d'autres mots de la phrase ou d'une phrase précédente.

\section{1- Pierre a volé Paul; il a porté plainte \\ 2- Jean a donné à Paul son cadeau}

Figure 14: Exemples d'erreurs à classer à l'intérieur de la catégorie 420: Référent.

Plusieurs erreurs sont faites à l'intérieur de cette sous-catégories et ce, la plupart du temps, parce que le scripteur ne connaît pas la règle: le mot référent doit référer au sujet de la phrase ou à celui de la phrase précédente. Évidemment, cette règle est une règle dite grammaticale; d'un point de vue sémantique, il pourra en être autrement. Or, pour un scripteur en apprentissage, il sera plus profitable de lui enseigner de choisir ses référents selon la règle grammaticale. La faute origine du fait que le ou les mots auxquels il est fait référence ne sont pas présents dans le texte. Il peut être possible, par exemple, d'apercevoir un pronom qui ne réfère à aucun mot dans le phrase (absent); ou la faute peut encore originer du 
fait que le mot référent renvoie à un autre mot dépourvu de logique (fautif). Ainsi, les fautes de référent ne sont pas non plus des fautes d'accord, mais bien plutôt le résultat d'une organisation fautive parce qu'agrammaticale en regard de la logique à exprimer.

La troisième sous-catégorie Mot manquant reçoit, pour sa part, les fautes tenant à l'absence d'un ou de quelques mots indispensables au sens ou à la grammaticalité de la phrase, par exemple de Je crains qu'il ( $\sqrt{ }$ ) soit parti. La structure exige tel mot et ce dernier manque. Généralement, les erreurs de cette sous-catégorie seront celles relatives à l'usage de la négation. D'autres cas seront ceux où il manque des pronoms, des déterminants et même des verbes à la phrase. En outre, même s'il apparaît net que la faute est de l'ordre de l'inattention, il importera quand même de la signaler au scripteur.

Là où tel mot manquait précédemment à la structure grammaticale, la sous-catégorie Mot superflu informera le scripteur que tel mot ne convient pas dans le cadre de la structure choisie. Ainsi, les mots inutiles, voire nuisibles à la phrase seront l'objet de la sous-catégorie Mot superflu comme les exemples (1) et (2) de la figure 15 en font état. Toutefois, lorsque le choix de mot est plutôt du type familier, une sous-catégorie Niveau de langue (650) de la catégorie Lexique (600) est disponible. Ce sera donc au correcteur de faire porter ses choix de classification selon la nature de l'erreur commise.

\footnotetext{
1- La différence d'avec la phrase de base

2-C'est pourquoi quiun athlète doit s'entrainer
}

Figure 15: Exemples d'erreurs à classer à l'intérieur de la catégorie 440: Mot superflu.

Tel sera aussi le cas pour la sous-catégorie Mot(s) mal choisi(s). Cette sous-catégorie englobe les erreurs touchant au mot ou à l'expression connue qui n'est pas utilisé dans la bonne circonstance, à titre d'exemple: Le sujet que je vous entretiens. Le correcteur de- 
vra une fois de plus être habile et bien différencier les fautes de mot(s) mal choisi(s) touchant la structure de la phrase (450) de celles de vocabulaire touchant le sens (600). L'erreur faite en sous-catégorie 450 touche la fonction du mot dans la phrase ce qui endommage ainsi toute la phrase. Cette erreur se veut contraire à celle de vocabulaire qui, elle, réfère plutôt à l'idée et au sens d'un mot.

La grille finale a décidé de faire un ajout à cet ensemble de sous-catégories proposé par la grille de base. Ainsi, la sixième et dernière sous-catégorie Construction fautive inventorie des erreurs de constructions qualifiées de phrases «qui n'ont pas d'allure». Le maître sait qu'il y a une ou des erreurs, mais il est incapable de la ou les cibler. La difficulté se veut trop grande, donc toute la phrase est à refaire. Ce sera le cas souvent ou de phrases incomplètes, ou de phrases mal ordonnées, ou ne correspondant pas aux lois logiques de constructions et qui, par le fait même, en affectent le sens ou la grammaticalité. Donc, la sous-catégorie Construction fautive est destinée à regrouper les erreurs affectant l'ensemble d'une phrase, les erreurs identifiables à un seul mot étant portées à la sous-catégorie précédente $\operatorname{Mot}(\mathbf{s})$ mal choisi(s).

Tout compte fait, le choix de pareilles sous-catégories est justifié par sa précision diagnostique. Les sous-catégories qui viennent d'être présentées sont tout au plus des catégories de surface regroupant plusieurs types d'erreurs. L'utilité de cette sous-catégorisation lui vient probablement de la simplification qu'elle fait d'un ensemble d'erreurs qu'il est impossible de détailler dans une grille, mais pour lesquelles une sous-catégorisation de surface permet de créer un air de famille constituant curieusement un bon outil d'intervention auprès des scripteurs, en raison du caractère de simplicité des sous-catégories retenues. 
Cette curieuse sous-catégorisation pourrait au demeurant se révéler avoir des liens précis et fort nets avec certaines des opérations du processus d'écriture qui mèneraient le scripteur à bien choisir ses référents, à ne pas omettre de mots, à ne pas en introduire d'inutiles, à en bien mesurer la place et le rôle, à bien les organiser dans l'ensemble de la phrase. Bien orthographier ses mots, bien les accorder et bien les situer dans l'ensemble d'une phrase nécessite aussi, et inévitablement, de choisir les signes de ponctuation appropriés dans l'écriture de ses phrases.

\subsubsection{La catégorie 500: Signes de ponctuation et conventions}

La cinquième catégorie Signes de ponctuation et conventions regroupe toutes les fautes de ponctuation, dont le point, la virgule et les conventions. Les sous-catégories et les sous-sous-catégories de cette catégorie sont présentées à l'intérieur de la figure 16.

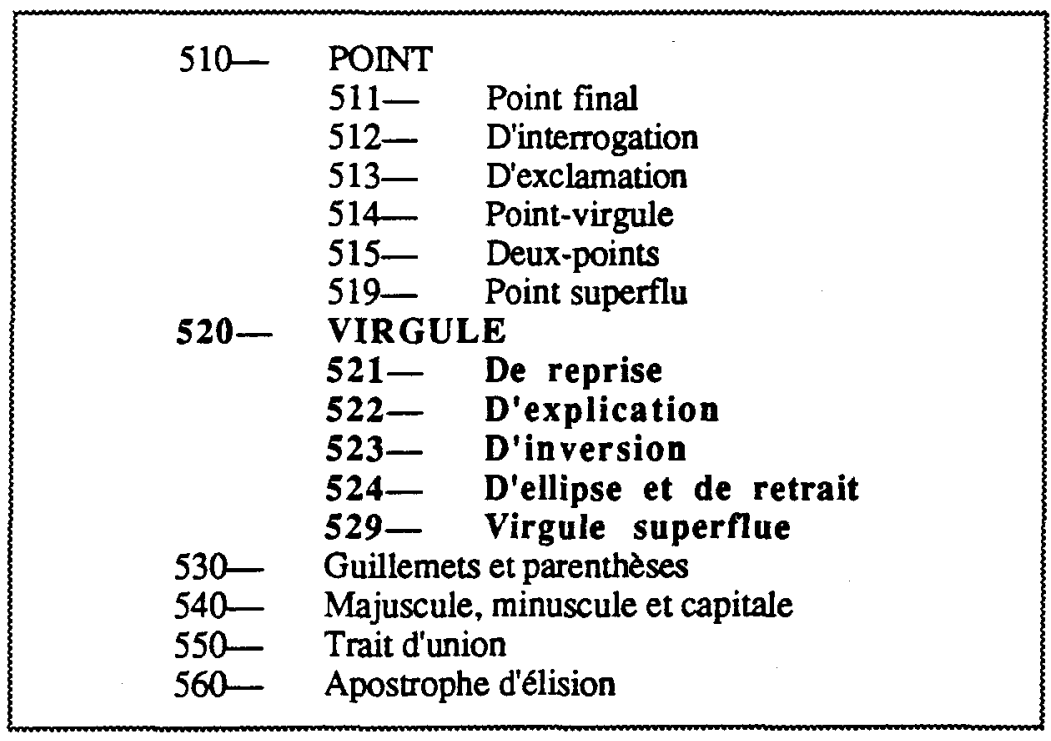

Figure 16: Sous-catégories et sous-sous-catégories de la catégorie 500: Signes de ponctuation et conventions.

L'observation des règles d'usage des signes de ponctuation et de conventions relatives à l'écriture d'un texte auront pour tâche de faire respirer, d'une part, les phrases et, 
d'autre part, l'ensemble du texte. Le scripteur sera ainsi mieux compris par celui qui le lira. Une catégorie de ce type sera utile pour le scripteur afin qu'il comprenne judicieusement la place et le rôle des signes de ponctuation qu'il utilise souvent, les deux principaux signes étant le point et la virgule. C'est ainsi que les deux premières catégories regroupent les différents types de points à utiliser, ainsi que les différents types de virgules.

La grille de base n'avait pas regroupé dans un ensemble les signes relatifs au point. La grille finale a donc apporté cette modification et ainsi ajouté une sous-catégorie Point superflu pour faire comprendre l'emploi inventé du point à certains endroits, comme le proposait d'ailleurs la sous-catégorisation des virgules. Étant donné l'aspect plutôt connu des différents emplois de points (final, d'interrogation, d'exclamation, point-virgule, deux-points), ils ne seront pas expliqués puis analysés, de même que les sous-catégories relatives aux conventions. Encore une fois, il est possible pour le lecteur de consulter le Guide de correction référentielle et interactive situé en annexe (voir annexe 1). Ce guide donne de nets exemples pour chacune des divisions de la grille, entre autres pour les souscatégories touchant les signes de ponctuation et les conventions présentées à l'intérieur de la catégorie Signes de ponctuation et conventions. Mais une sous-catégorie à laquelle il faut porter de l'intérêt et ce, pour sa nouveauté et sa grande portée diagnostique et d'apprentissage, est celle regroupant les cas d'emploi de la virgule.

Dans la plupart des grilles, il n'est pas fait de distinction des virgules, mais plutôt une seule catégorie classe l'ensemble des cas possibles. Pour leur part, Grevisse (1980) et Goosse (1986) dans le Bon usage présentent différents cas d'emploi de la virgule. Toutefois, le relevé qui en est fait, même s'il est détaillé, ne constitue pas un guide satisfaisant pour le scripteur cherchant à utiliser adéquatement ce signe de ponctuation. 
Par ailleurs, il a souvent été enseigné de placer une virgule lorsque le besoin de faire une pause se fait sentir. Une règle qui ne manque pas d'être insuffisante et qui fait courir beaucoup de risques d'erreurs. Pour maîtriser l'usage de ce petit signe fort complexe qu'est la virgule, il importe, d'une part, de maîtriser et de distinguer les emplois à faire de la virgule et, d'autre part, d'en comprendre, malgré les difficultés, la logique.

Ainsi, à partir d'analyses grammaticales, quatre classes majeures d'emplois de la virgule ont été identifiées: Virgule de reprise, Virgule d'explication, Virgule d'inversion et finalement Virgule d'ellipse et de retrait. Les expérimentations ont montré que le recours à ces classes permet un enseignement plus systématique et plus efficace que celui que permet les inventaires des grammaires. Comme c'était un objectif majeur de construire une grille reliée aux apprentissages à faire ou encore menant à des apprentissages, une classification rattachée à l'enseignement ou à une grammaire bien structurée, s'imposait. Les exemples proposés pour chacune des sous-catégories ont pour objectif d'illustrer chacun des cas déterminés. Toutefois, il importe de considérer ces exemples, cette fois-ci, non pas comme des erreurs-types, mais bien comme des emplois corrects d'utilisation.

D'abord les deux premiers exemples (1) et (2) de la figure 17 illustrent des cas d'emplois de la virgule de reprise. La virgule se veut de reprise, car elle marque le retour, qu'elle introduit, d'éléments de même nature. Elle vaut pour les mots, elle vaut aussi pour les propositions.

1- Le français, les mathématiques et la géographie sont les matières que je préfere.

2- Je travaille trop, je n'ai pas de temps pour mes loisirs.

3- Bernard, qui attendait son ami, fut surpris par la tempête.

4 - Je ne peux oser faire ce geste, car les conséquences pourraient en être néfastes.

Figure 17: Exemples d'erreurs à classer à l'intérieur des sous-sous-catégories 521 - Virgule de reprise et 522 - Virgule d'explication. 
La virgule d'explication (exemples 3 et 4), quant à elle, vient séparer tout groupe de mots ou propositions ayant une valeur explicative. Pour les propositions, elle sert à signaler la valeur explicative des conjonctions de coordination autres que les conjonctions de reprise et, ou, ni.

La virgule peut-être la plus riche dans son rôle et ses effets est la virgule d'inversion. Les exemples (1) et (2) de la figure 18 font voir des emplois de ce troisième type de virgule.

1- Après le dîner, les jeunes étaient allés danser.

2-Avant qu'il n'arrive, je pleurais.

Figure 18: Exemples d'erreurs à classer à l'intérieur de la sous-sous-catégorie 523-Virgule d'inversion.

Logiquement, la virgule d'inversion souligne les effets d'expressivité du discours qu'elle s'emploie à marquer. Ce faisant, elle signale les contorsions grammaticales de la phrase et souligne la fonction de certaines contorsions. Généralement ce type de virgule sera utilisé pour marquer l'inversion de structures, souvent des compléments circonstanciels et des propositions circonstancielles, dans une phrase. L'apprenant scripteur n'arrivera à maîtriser cet emploi de virgule qu'en se donnant concurremment la maîtrise des règles de structuration grammaticale et logique de la phrase.

Finalement, les trois derniers exemples $(1,2$ et 3$)$ de la figure 19 font voir des cas du quatrième type de virgule: D'ellipse et de retrait. Ce type d'emploi de la virgule est utilisé pour placer certains mots ou groupes de mots en retrait. Il sert à isoler un ou des mots mis en apostrophe ou à marquer l'élision d'un mot, la plupart du temps d'un verbe. 
1-Chien, rentre dans ta niche!

2- Il me plaît, voyez-vous, d'assister à ce spectacle.

3- Le vent était doux et le soleil, brûlant.

Figure 19: Exemples d'erreur à classer à l'intérieur de la sous-sous-catégorie 524 - Virgule d'ellipse et de retrait.

Or, les apprentissages et les connaissances auxquels réfêrent ces emplois de virgule d'ellipse et de retrait sont évidents. Les erreurs indiqueront aux maîtres et à l'élève, encore une fois, où faire porter leurs efforts.

Lors des expérimentations de correction, il a été possible de constater qu'à la longue, l'apprenant scripteur en vient à distinguer un emploi de virgule particulier, à prendre nettement conscience de la difficulté qui est sienne et à s'avancer en bonne motivation dans un travail de correction et d'apprentissage. Et ce, jusqu'à maîtriser les différents cas d'emploi.

Les catégories se rapportant aux règles de base d'écriture ont maintenant été parcourues. Les prochaines catégories font intervenir, pour leur part, les finesses d'écriture de la langue. Elles sont d'un autre niveau, niveau qui permet de donner une toute autre qualité au texte. D'abord la catégorie Lexique, issue principalement de la pragmatique, aidera le scripteur à corriger ses emplois de vocabulaire fautifs en regard de leur signification. La plupart des grilles présentent une catégorie Lexique, la maîtrise du vocabulaire étant un des objectifs majeurs du programme de français.

\subsubsection{La catégorie 600: Lexique}

La catégorie Lexique renferme des sous-catégories qui permettront d'indiquer clairement au scripteur ses emplois fautifs dans le choix de ses mots. Il est rare que les règles 
d'usage des mots de vocabulaire fassent l'objet d'un enseignement systématique. C'est pourquoi la catégorie sera utile, d'une part, pour le maître voulant indiquer à son scripteur les emplois de mots n'étant pas acceptés par la langue française et, d'autre part, pour le scripteur qui apprendra en pratique à utiliser des termes justes et adéquats. Le jugement porté par le correcteur dans les choix de mots de ses scripteurs est, dans plusieurs cas, une affaire de goût. Or, certaines règles sont véhiculées, par exemple celles se rapportant aux anglicismes. La langue évolue et porte ses choix sur certains mots et en refuse d'autres; c'est ainsi qu'il importe d'être vigilant et de corriger à l'écrit ces emplois inacceptables en français.

La grille originelle ne proposait que deux sous-catégories: Impropriété et Anglicisme. Comme il est possible de le constater, à l'aide de la figure 20, la grille finale propose trois nouvelles sous-catégories: Accord conceptuel, Barbarisme, pléonasme et tautologie et Niveau de langue. La catégorie Lexique de la grille finale semble ainsi, par cette nouvelle division, rassembler et ce, d'après les expérimentations, des sous-catégories précises et traduisant bien les différentes difficultés des scripteurs, rattachées au vocabulaire.

$\begin{array}{ll}610- & \text { Impropriété } \\ 620- & \text { Accord conceptuel } \\ 630- & \text { Anglicisme } \\ 640- & \text { Barbarisme, plénasme et tautologie } \\ 650- & \text { Niveau de langue }\end{array}$

Figure 20: Sous-catégories de la catégorie 600: Lexique.

La catégorie Lexique entretient parfois des liens fort étroits avec d'autres catégories de la grille avec, par exemple, celles de Structure de la phrase et de Stylistique. Le correcteur devra ainsi se méfier dans son classement des fautes et ne considérer que les fautes de lexique comme des fautes de lexique. Une erreur qui est souvent faite est celle de classer des termes impropres (sous-catégorie 610) à l'intérieur de la sous-catégorie Mot(s) mal 
choisi(s) (450) relevant de la catégorie Structure de la phrase. Les mots mal choisis dans leur fonction seront des cas de Structure de phrase, mais ceux mal choisis dans leur signification seront plutôt à considérer en catégorie Lexique. Les exemples de la figure 21 montrent justement des erreurs d'impropriété: ce sont des mots mal choisis, mais dans le sens qu'ils portent.

1 - Elle le joint (rejoint) à $19 \mathrm{~h} 00$.

2- Le lac est creux (profond).

Figure 21: Exemples d'erreurs à classer à l'intérieur de la sous-catégorie 610 Impropriété.

La sous-catégorie 610 - Impropriété regroupe les erreurs relatives aux emplois impropres de un ou plusieurs mots ne traduisant pas, de façon satisfaisante, le sens recherché ou attendu. Pour sa part, la sous-catégorie Accord conceptuel compte des erreurs pour lesquelles un mot ne s'accommode pas conceptuellement avec le ou les autres mots utilisés dans la phrase. Ce que montre les exemples (1), (2) et (3) de la figure 22.

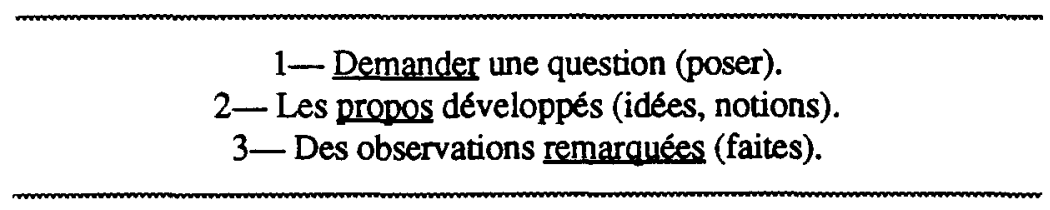

Figure 22: Exemples d'erreurs à classer à l'intérieur de la sous-catégorie 620-Accord conceptuel.

Cette sous-catégorie, Accord conceptuel, sera utile pour faire prendre conscience des illogismes d'accord entre deux mots, parfois imperceptibles, ou plutôt méconnus par le scripteur. Seules les notations et le souci constant du correcteur permettront de réaliser la nuance à faire jouer dans l'accord conceptuel de deux mots ne s'accommodant pas ensemble.

La prochaine sous-catégorie: 630 - Anglicisme est connue. Elle englobe les erreurs touchant ou les termes dealer, ou les expressions jusqu'd date, ou les mots gang emprun- 
tés à la langue anglaise. La tentation est grande pour les scripteurs, surtout ceux de niveau secondaire, d'utiliser des anglicismes. Langue proche voisine et de communication, l'anglais s'impose parfois au français et y laisse des traces. À une certaine époque de l'évolution du français, certains mots de l'anglais ont été empruntés, ont pris leur place et se sont légitimisés. Or, il convient malgré tout de filtrer certaines constructions ou emplois inacceptables en français. Il importe aussi de faire connaître au scripteur, à l'aide de cette sous-catégorie, la nature d'anglicisme de ses mots ou expressions. Cela n'aura pour effet que d'encourager le scripteur à utiliser des mots corrects et propres à la langue française.

La sous-catégorie 640_ Barbarisme, pléonasme et tautologie peut parfois être confondue avec la sous-catégories 720 - Concision de la langue relevant de la catégorie 700 de Stylistique. Ce sera, encore une fois, au maître de juger de quelle façon il veut transmettre la difficulté à son élève. Une erreur classée en catégorie Stylistique sera considérée comme étant une erreur de plus haut niveau. Les exemples de la figure 23 illustrent les emplois de Barbarisme, de pléonasme et de tautologie.

1- Inormal
2 - Se virer de bord
$3-$ Se tasser de la porte
$4-$ Monter en haut

Figure 23: Exemples d'erreurs à classer à l'intérieur de la sous-catégorie 640: Barbarisme, pléonasme et tautologie.

L'emploi d'un barbarisme sera celui d'un mot ou inventé, ou déformé (exemple 1), ou détourné du sens qu'il porte habituellement (exemples 2 et 3). Le pléonasme et la tautologie, pour leur part, seront considérés comme des mots superflus ou redondants (exemple 4).

La dernière sous-catégorie 650- Niveau de langue se devait d'être ajoutée à cet ensemble des sous-catégories relevant de la catégorie Lexique. Les erreurs faites à cette 
sous-catégorie seront celles des emprunts de la langue orale utilisés en langue écrite. Certains emplois ou expressions de l'oral sont inacceptables à l'écrit. La sous-catégorie sera utile, entre autres, afin de signaler ces emplois de niveau familier, ainsi que des constructions issues de l'oral, par exemple la répétition des pronoms et les constructions plutôt familières, tels que présentés à la figure 24. Le niveau accepté en écriture est davantage un niveau correct ou soigné et, peut-être même à la rigueur, soutenu.

1- Le français, moi, je l'écris mal.

2- Je sais pas si je peux aller là.

3-C'est correct de penser à ça.

Figure 24: Exemples d'erreurs à classer à l'intérieur de la sous-catégorie 650: Niveau de langue.

Tout compte fait, la catégorie Lexique se révèle essentielle à l'intérieur d'une grille de correction. D'ailleurs la plupart des grilles proposent aussi une catégorie de ce type permettant ainsi au scripteur de corriger ses erreurs lexicales. L'emploi des termes justes présuppose évidemment une écriture de meilleure qualité. C'est ainsi que cette catégorie amorce adéquatement celle de stylistique. Car choisir le vocabulaire qu'il convient, c'est déjà donner à son écriture une qualité, un style. Or, dans ce style, toute la pensée entre en jeu, de même que l'organisation du texte. Tout est dans la façon de transmettre l'idée. Les trois dernières catégories, référant davantage au fond d'un texte, seront traitées sous une même analyse.

\subsubsection{Les catégories 700,800 et 900 : Stylistique, Qualité textuelle et Qualité de la pensée}

Des catégories pour lesquelles le choix des sous-catégories présente de grandes difficultés sont les catégories relatives au fond d'un texte: Stylistique, Qualité textuelle et 
Qualité de la pensée. En effet, le champ d'habiletés auquel réfèrent ces catégories ne présente pas une organisation interne très nette, peut-être quelque peu pour la catégorie 800 Qualité textuelle, mais non pas comme ce pouvait être le cas pour la plupart des catégories précédentes, qui trouvaient dans la grammaire et même la linguistique des fondements de référence.

C'est conséquemment dans une démarche pragmatique que les sous-catégories des catégories de fond de la grille, principalement de Stylistique et de Qualité de la pensée, ont été établies. Pour sa part, la catégorie Qualité textuelle s'est construite en référence aux ouvrages traitant d'organisation des textes dont Brousseau et al. (1991) et Garneau (1993), entre autres. Les notions de cette catégorie sont relativement connues et exploitées depuis nombre d'années.

La stylistique se définit communément comme l'art de bien écrire. Albalat (1992, p.45) définit le style comme étant «[...] la manière propre à chacun d'exprimer sa pensée par l'écriture ou la parole». Pour Marouzeau (1963, p.17) le style est «[...] la mise en oeuvre méthodique des éléments fournis par la langue». La langue est très présente dans le style, car les erreurs de style réferent fréquemment à des difficultés de langue, par exemple à celles de la morphologie, de la syntaxe, du vocabulaire et même de l'organisation des mots dans une phrase. Ainsi dans la stylistique, l'entier du domaine de la langue est couvert, mais le style, comme le souligne Albalat (1992, p.45), «[...] comprend le fond et la forme». La grille finale considère la catégorie Stylistique comme une catégorie de fond. Or, cette catégorie ne l'est pas essentiellement. Les concepteurs de la grille finale la situent à vrai dire à mi-chemin entre la forme et le fond. De fait, la catégorie Stylistique se veut être une catégorie conclusive à la forme et introductive au fond. Car répondre 
à des lois de style, c'est autant répondre à des lois de transmission logique des idées qu'à celles d'observation des règles d'écriture.

Les théories de stylistique abondent, mais elles sont à la fois diverses et semblables. À quoi s'ajoute qu'elles évoluent, ou du moins sont remises périodiquement en cause, sans jamais qu'une approche ou une interprétation ne s'impose conclusivement. Essentiellement, pour Mottoulle (1957), cinq qualités majeures réferent au style: la correction de style, sa précision, sa concision, son harmonie et son efficacité. Albalat (1992) réduit, pour sa part, à trois termes recouvrant ainsi, pour lui, l'entier du style: l'originalité, la concision et l'harmonie.

Il convenait donc, pour une grille de correction, de ne pas multiplier les sous-catégories, de façon à conserver à chacune son champ propre. Ce sont principalement les traités de Mottoulle (1957) et d'Albalat (1992) qui ont mené par recoupements à identifier et à retenir trois sous-catégories distinctes et complémentaires, couvrant ainsi l'entier du champ des habiletés stylistiques. Il convenait, de plus, que les sous-catégories choisies se complètent les unes des autres. La figure 25 montre ces sous-catégories, pour lesquelles l'élaboration a été délicate.

$$
\begin{aligned}
& 710 \text { - Précision de la langue } \\
& 720 \text { - Concision de la langue } \\
& 730 \text { - Harmonie de la langue }
\end{aligned}
$$

Figure 25: Sous-catégories de la catégorie 700: Stylistique.

Ainsi, en correction, cibler une erreur de stylistique sera possible à l'aide de ces sous-catégories qui identifieront trois qualités majeures d'un style: la précision, la concision et l'harmonie. La sous-catégorie Précision de la langue agira principalement pour 
informer le scripteur si sa langue est claire, si elle porte bien le propos, si les mots sont bien choisis et si les termes sont précis. Les erreurs de cette sous-catégorie font souvent référence à des phrases ou des mots incompréhensibles, embrouillés, bref imprécis. La deuxième sous-catégorie, pour sa part, vérifiera la concision et le netteté de la langue. Généralement, les fautes contenues à l'intérieur de cette sous-catégorie seront de l'ordre d'une langue compliquée, manquant de concision, de simplicité ou présentant une redondance. Finalement, la qualité majeure du style voudra atteindre une langue belle, coulante, agréable, originale; bref, harmonieuse. Cette sous-catégorie Harmonie de la langue, sera donc utile afin que le correcteur indique la lourdeur de certains passages, de même que la langue ennuyante, gênante. L'harmonie de la langue tente de relever le style bizarre ou l'écriture maladroite, ainsi que certaines erreurs de disposition et d'ordre des mots.

Entre les trois termes retenus (précision, concision, harmonie), il est apparu les rapports d'un triptyque: l'harmonie étant le résultat final qui ne peut être atteint hors la précision et la concision de la langue, tandis que la précision se présente comme une qualité préliminaire, condition d'un style concis. La figure 26 montre ce rapport d'un triptyque, élaboré sous l'influence de l'intuition et de la pratique.

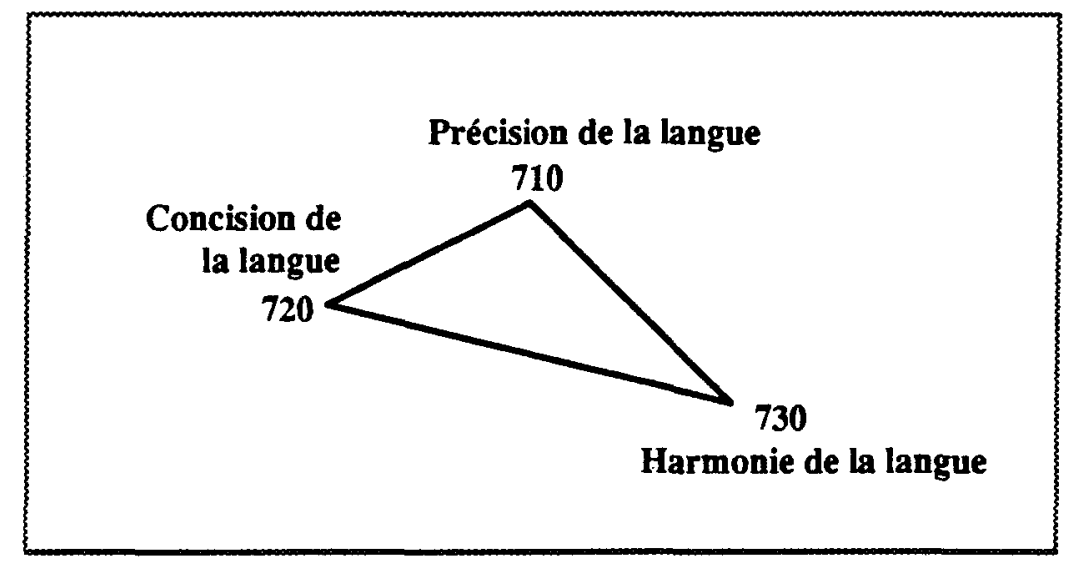

Figure 26: Organisation en triptyque de la catégorie 700: Stylistique. 
Cette proposition ne manquera pas d'être l'objet de vives critiques. Toutefois, cette taxinomie mérite que la pratique en soit tentée, car les expérimentations auprès d'étudiants universitaires ont donné satisfaction. Les grilles les plus communément en usage réduisent à deux termes ces catégories: la pratique en est une de simplification acceptable. Toutefois, l'intérêt de l'approche en triptyque ne manquera pas, sur la foi des expérimentations, d'apporter satisfaction à qui voudra bien l'utiliser.

L'organisation en triptyque de la catégorie Stylistique est apparue être une solution valable. De ce fait, l'application en a aussi été tentée aux deux catégories restantes de la grille: Qualité textuelle et Qualité de la pensée; deux catégories se prêtant aussi à une analyse pragmatique, surtout la dernière. La catégorie 800 (Qualité textuelle) réfère donc en 810 au respect des règles d'organisation d'un texte, en 820 au respect des règles de soutien, et en 830 au respect des règles du genre. La dernière sous-catégorie 830- Respect du genre est semblable à celle de la sous-catégorie 730: Harmonie de la langue, sous-catégorie conclusive de la catégorie 700 . La figure 27 montre le rapport en triptyque aussi retenu pour la catégorie $800-$ Qualité textuelle.

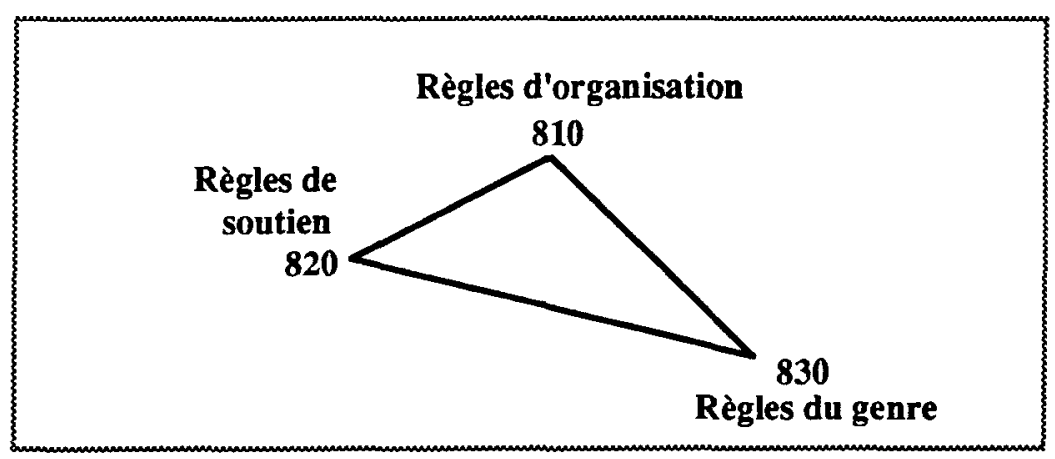

Figure 27: Organisation en triptyque de la catégorie 800: Qualité textuelle.

Les sous-sous-catégories de la catégorie 800 sont présentées à l'intérieur de la figure 28 . Elles ne feront pas l'objet de développements plus approfondis tellement ces notions de construction d'un texte sont connues et évidentes. 


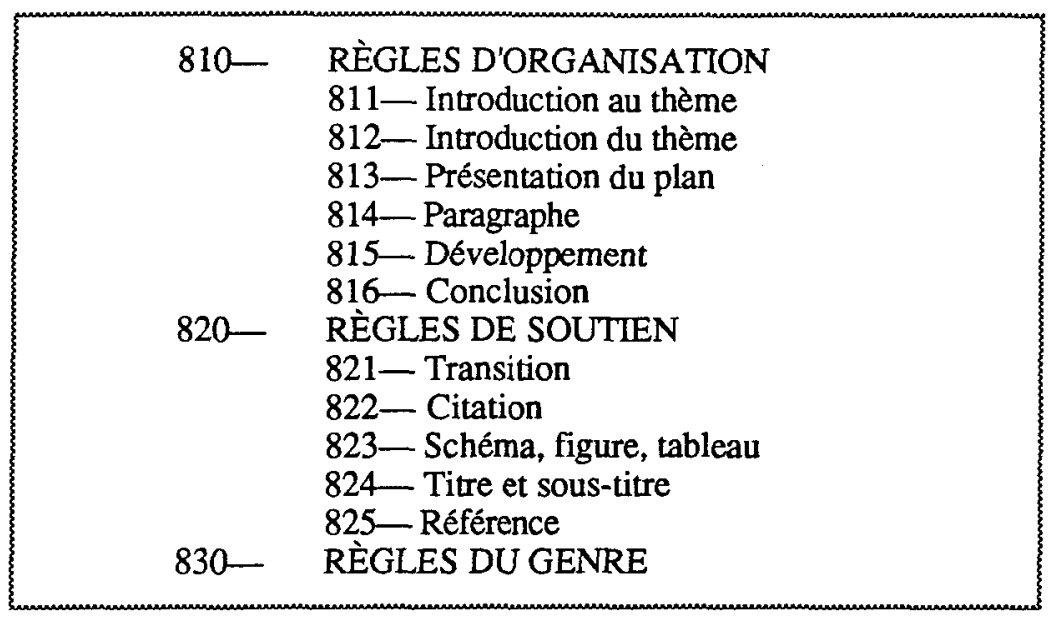

Figure 28: Sous-catégories et sous-sous-catégories de la catégorie 800: Qualité textuelle.

Pour sa part, la dernière catégorie de la grille: 900 — Qualité de la pensée est présente afin de coter la qualité des idées et des propos présentés dans un texte. Or, les normes d'usage afin de présenter ses idées sont difficilement ciblées tellement les possibilités sont larges. Ce sera au correcteur de délimiter avec ses étudiants quelles idées sont acceptables pour le niveau donné et lesquelles ne le sont pas. Cette catégorie examine en 910 le choix et la netteté des idées, en 920 la logique et l'enchaînement des idées, en 930 la profondeur et l'originalité des idées.

La grille finale se devait de contenir une catégorie Qualité de la pensée, car cette catégorie est, malgré tout, le critère fondamental d'évaluation d'un texte. Même si elle est une affaire de goût, l'évaluation des idées peut se faire à l'aide des trois sous-catégories proposées, élaborées en pragmatique et présentées à la figure 29.

\section{0- Choix et netteté des idées \\ 920-Logique et enchaînement des idées \\ 930 - Profondeur et originalité}

Figure 29: Sous-catégories de la catégorie 900: Qualité de la pensée. 
La première sous-catégorie $910-$ Choix et netteté des idées touche l'orientation du sujet. Comme point de repère, le correcteur devra juger si le sujet est bien choisi en rapport à celui proposé et si les idées sont nettes, claires et précises. De plus, si le texte répond à la question, s'il est exempt d'inexactitudes théoriques et si tous les points à traiter sont couverts. La sous-catégorie 920— Logique et organisation des idées fera référence à l'articulation du texte. Le correcteur s'attaquera davantage à la qualité du développement, à l'articulation du raisonnement et à la logique d'ensemble du texte. Finalement, l'originalité d'un texte tient souvent à la maîtrise des idées et du contenu. Le correcteur doit juger si le texte fait la preuve d'une grande compréhension, si le texte comporte des développements originaux et si le texte fait la preuve d'une grande maîtrise du contenu et de la pensée, ce que permettra la troisième sous-catégorie de la grille: Profondeur et originalité.

S'il y avait une seule sous-catégorie à retenir pour les qualités relatives au fond d'un texte, ce serait la dernière sous-catégorie de la catégories 900, soit Profondeur et originalité. La grille se termine sur une qualité transcendante d'un texte, laquelle mesure le summum de la qualité d'un texte. Tout comme les catégories 700 et 800 , la catégorie 900 possède sa catégorie conclusive de Profondeur et d'originalité. Cette dernière catégorie se présente aussi sous l'oeil d'un triptyque, ce que permet de constater la figure 30.

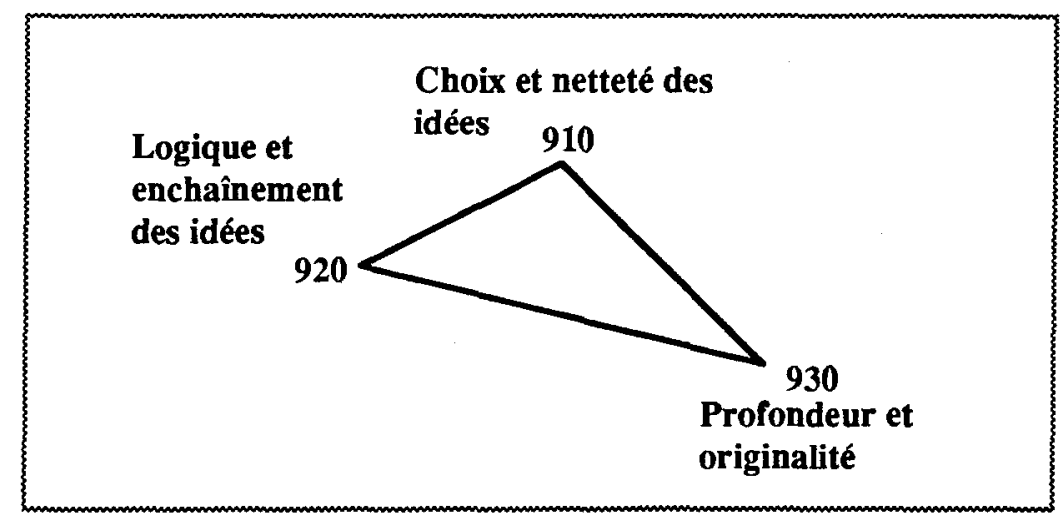

Figure 30: Organisation en triptyque de la catégorie 900: Qualité de la pensée. 
Voilà la grille élaborée dans le cadre d'intérêts pratiques et, par le fait même, qui est utilisée depuis quelques années pour corriger les productions écrites des étudiants universitaires, essentiellement une clientèle de futurs maîtres. Un premier problème à la recherche était celui cherchant à établir l'utilité d'une grille en correction, plus précisément son rôle et sa composition. L'étude montre que la grille force à une nette identification des fautes. Lorsque la grille est bien établie, elle force à bien analyser l'erreur commise. L'enseignant se trouve donc en meilleure position de travailler avec son apprenant. Vue de cette façon, la grille possède deux rôles: elle est un bon outil diagnostique et un bon outil d'apprentissage.

La grille se veut un outil diagnostique pour le maître qui cherche à cibler les difficultés de ses élèves en écriture. Elle permet de guider le correcteur dans sa correction et les sous-catégories permettent, pour leur part, de mettre le doigt sur des habiletés non-maîtrisées. Le maître saura ainsi où faire porter ses efforts en enseignement et ses leçons se verront mieux orientées et ce, après avoir ciblé les difficultés majeures de ses apprenti scripteurs.

La grille se veut aussi un outil d'apprentissage pour l'élève, car les différentes cotes attribuées à sa copie le référeront à des sous-catégories de la grille, donc à des théories explicatives englobantes. En sachant la nature exacte de sa faute, l'élève a plus de chance d'en mémoriser la nature, ainsi que la règle rattachée à l'erreur. L'élève saura donc où se référer pour corriger ses difficultés et à la longue, à la suite de plusieurs pratiques d'écriture et de correction, il en viendra à maitriser les différents aspects proposés par la grille.

Le deuxième chapitre a eu pour objet, la grille. Ses neufs catégories ont été situées et expliquées à l'aide d'exemples de classification, de théories pour certains cas, et de données issues de la pragmatique pour d'autres. L'importance d'utiliser la grille a été montrée 
et ce, entre autres, pour la précision du diagnostic qu'elle permet d'établir. Il conviendra, toutefois, d'adopter la grille au niveau scolaire visé et aux intérêts des correcteurs étant donné que celle présentement proposée est de haut niveau. De plus, si cette grille reste perfectible sur certains points, elle ne manquera pas de donner des résultats satisfaisants aux utilisateurs: le maître et l'élève. Ces derniers étant grandement gagnants d'utiliser cette grille, car pour l'un, elle se veut un excellent outil diagnostique et, pour l'autre, un outil précieux d'apprentissage. À certaines conditions évidemment et si certains pièges grossiers d'utilisation sont évités.

Car, en réalité, une grille utilisée seule ne peut pas avoir grand effet sur la poursuite de l'amélioration en français écrit. Le troisième chapitre viendra donner réponse au deuxième problème du départ qui cherche à préciser la façon adéquate d'utiliser la grille. Le choix s'est arrêté sur l'évaluation formative. Pour ce faire, les théories de l'évaluation formative seront traitées et rapprochées des théories cognitives de l'apprentissage. Des principes de correction formative seront proposés et discutés; ils auront pour but de venir guider l'utilisation même de la grille. Car au fond, la question est moins d'utiliser une grille, quelle qu'en soit la qualité, que d'aborder selon de sains principes pédagogiques et didactiques l'activité de correction de la production écrite et de formation de l'apprenti scripteur. 


\section{CHAPITRE III}

L'évaluation formative des productions écrites 
Il fut question au début des travaux de recherche d'élaborer une grille qui, par ellemême - ou presque - , permette une pratique corrective qui soit efficace en terme d'apprentissage. Or, il est apparu à l'expérimentation que si la grille peut être un outil diagnostique de qualité et un outil d'apprentissage efficace, ses effets varient en fonction du respect de certaines conditions d'utilisation. En effet, la grille est un outil diagnostique de qualité à la condition que le diagnostic s'inspire d'objectifs formatifs plutôt que d'objectifs sommatifs. De même, la grille est un outil d'apprentissage efficace à condition de s'intégrer dans une approche formative d'apprentissage.

Ce dernier chapitre procède à une analyse théorique de l'évaluation formative, puis à un rapprochement de l'évaluation formative avec les théories cognitives. Une troisième partie présente, sur la base de ces analyses, des principes de correction formative destinés à guider les maîtres dans leurs pratiques de correction des productions écrites.

\subsection{L'ÉVALUATION FORMATIVE}

Au moment où dans les milieux scolaires on était à la recherche de renouveau, de méthodes et de stratégies nouvelles d'enseignement et d'apprentissage, l'évaluation formative s'est imposée dans les moeurs pédagogiques. Cette nouvelle pratique d'évaluation, centrée sur la progression de l'apprenant, se définit comme étant un régulateur des apprentissages, c'est-à-dire un guide de formation pour l'apprenant impliqué dans un processus d'apprentissage. Elle vise l'amélioration des apprentissages et le suivi constant en vue d'une progression. L'idée de l'évaluation formative, d'abord introduite par Scriven au cours des années soixante, puis poursuivie par Bloom au cours des années soixante-dix et 
reprise par Scallon (1988) dans un ouvrage fort répandu pose comme principe de se soucier prioritairement de l'apprenant et de sa progression en pratique évaluative. Scallon (1988, p.3) écrit d'ailleurs de l'évaluation formative qu'elle «relève d'abord et avant tout d'un souci constant de la part des enseignants d'être à l'affût des moindres difficultés de parcours, afin de corriger, réorienter, ou améliorer avant qu'il ne soit trop tard». L'aspect le plus important en évaluation formative ne sera pas le jugement ou l'appréciation donnée à la copie, mais bien cette régulation des apprentissages. À ce propos, Scallon (1988, p.26) dira que «ce n'est pas le jugement qui est le terme d'un processus, mais bien une action concrète à poser».

L'objet de l'évaluation formative est donc l'élève et ses apprentissages. À l'intérieur de cette pratique, le maître doit exercer un souci constant à l'égard des difficultés de l'élève, pour ensuite mieux intervenir. Car tout enseignant a comme préoccupation première que son ou ses apprenants réalisent des apprentissages. Amener ses élèves ou ses étudiants à progresser, à faire des apprentissages, là est le but de l'évaluation formative. Enfin, il fait peu de doutes, même si peu ou pas d'auteurs n'ont montré ces rapports, que l'évaluation formative origine de la pensée cognitive et qu'elle s'inscrit dans une approche cognitive d'apprentissage.

\subsection{L'ÉVALUATION FORMATIVE ET LE CADRE COGNITIF}

L'évaluation formative a donc pour but de réguler l'apprentissage. Or, l'évaluation formative n'a jamais montré clairement qu'elle origine de l'approche cognitive. Elle pourrait prendre pourtant tout son sens, par exemple à l'intérieur des théories cognitives de Gagné (1976). Les théories cognitives de cet auteur avaient, dans leurs développements, la préoccupation profonde de guider constamment les apprentissages des élèves inscrits 
dans un processus d'apprentissage. La notion d'évaluation formative est présente à l'intérieur des théories de cet auteur, même si elle n'y est pas nettement développée. À l'intérieur du processus d'enseignement-apprentissage, les phases de performance et de renforcement de Gagné permettent de faire un rapprochement avec les propos de l'évaluation formative. La démonstration mérite d'être faite.

\subsubsection{Le renforcement formatif dans une approche cognitive renouvelée}

Gagné (1976) affirme que tous les élèves d'un groupe le moindrement homogène, au primaire du moins, peuvent et doivent réussir les apprentissages proposés à l'ensemble du groupe; et que, de plus, si une évaluation est orientée en tenant compte du cheminement et des réalisations des apprenants, elle peut avoir un effet bénéfique sur l'apprentissage.

Gagné propose un processus d'enseignement-apprentissage par découverte distinguant en tout huit phases: de motivation, d'appréhension, d'acquisition, de rétention, de rappel, de généralisation, de performance et de feed-back. Toutefois Roy (1995), sous l'effet de la pratique, a modifié légèrement les données du processus d'enseignementapprentissage de Gagné et c'est à ce modèle renouvelé qu'il est choisi de référer, parce que plus explicite et plus net dans ses descriptions. Roy propose, plutôt que huit, dix phases au processus. La figure 31 inventorie et situe les phases de Roy (1995), ainsi que leurs événements d'enseignement-apprentissage, pour l'apprentissage d'une règle en habileté intellectuelle. 


\begin{tabular}{|c|c|}
\hline Phases du processus & $\begin{array}{c}\text { Événements d'enseignement-apprentissage } \\
\text { d'une règle logique }\end{array}$ \\
\hline 1. MOTIVATION LOINTAINE & Créer ou réactiver la motivation. \\
\hline 2. MOTIVATION SPÉCIFIQUE & Présenter la performance attendue. \\
\hline 3. ATTENTION GÉNÉRALE & S'assurer de l'attention de l'apprenant. \\
\hline 4. ATTENTION SPÉCIFIQUE & Assurer le rappel des préalables. \\
\hline 5. ACQUISITION & $\begin{array}{l}\text { Placer en situation de découverte; fournir des indices } \\
\text { croissants jusqu'à découverte. }\end{array}$ \\
\hline 6. RÉTENTION À COURT TERME & Faire produire l'énoncé de la règle. \\
\hline 7. PERFORMANCE & Vérifier la maîtrise en situation de performance. \\
\hline 8. RENFORCEMENT & Présenter un renforcement formatif. \\
\hline 9. GÉNÉRALISATION & $\begin{array}{l}\text { Proposer en autonomie d'apprentissage des performances de } \\
\text { niveau supérieur. }\end{array}$ \\
\hline 10. RÉTENTION À LONG TERME & $\begin{array}{l}\text { Prévoir les besoins de pratique pour le futur et les techniques } \\
\text { de rappel à utiliser. }\end{array}$ \\
\hline
\end{tabular}

Figure 31: Processus et événements d'enseignement-apprentissage d'une règle en habileté intellectuelle (Roy, 1995).

Le modèle est hiérarchique, car chacune des phases est, théoriquement du moins, préalable à la suivante. La maîtrise du contenu d'une phase nécessite la maîtrise du contenu de la phase précédente. Le processus a pour objectif de rendre l'apprenant maitre de son apprentissage, l'enseignant devenant ainsi pour l'apprenant un guide. Tout comme pour l'évaluation formative, l'apprenant est respecté dans son cheminement, ainsi que dans le rythme de son apprentissage.

Dans le processus d'enseignement-apprentissage par découverte de Gagné (1976) tel que retouché par Roy (1995), les phases de performance et de renforcement font état de préoccupations semblables à celles des théories de l'évaluation formative, c'est-à-dire de «Vérifier la maîtrise en situation de performance» et de «Présenter un renforcement formatif». Théoriquement, la phase 7 de performance est la mise en application des apprentissages réalisés, afin d'en vérifier la maîtrise. Dans cette phase, le maître donne à l'apprenant 
l'occasion de mettre en pratique les connaissances acquises. Or, pour que les apprentissages se solidifient, il est nécessaire qu'une évaluation de la performance soit faite par l'enseignant et c'est ce qu'exige la phase 8 de renforcement.

Le rôle que viendra jouer la phase de renforcement, en sera un d'évaluation de la performance de l'apprenant; cette phase 8 de renforcement se définit comme suit dans le modèle modifié de Roy: «Présenter un renforcement formatif». La phase de renforcement se greffe donc très étroitement sur celle de performance et a pour but principal de fournir à l'apprenant une mesure de sa réalisation, mais une mesure inscrite dans un cadre formatif. Ce qui signifie que la mesure n'est pas absolue, qu'elle tient compte de l'apprenant, de ses progrès, de son cheminement d'apprentissage. L'apprenant sera renforcé pour avoir fait le bout de chemin que ses capacités lui permettaient. Il appartiendra au maître de juger quoi présenter de la route qui reste à parcourir, et comment le présenter. Au total, le meilleur renforcement formatif est celui qui rejoint les phases 1 et 2 de motivation lointaine et de motivation spécifique, relançant l'apprenant vers des compléments d'apprentissage ou d'autres apprentissages.

La notion d'évaluation formative se trouvait donc dans les théories cognitives et se retrouve dans leurs développements. La notion apparaît d'ailleurs plus nette dans ses effets et dans sa nature lorsqu'elle est examinée dans le cadre d'une démarche complète d'enseignement-apprentissage, ainsi qu'il vient d'être fait, et se retrouvera dans les appellations d'approche formative, de cadre formatif, d'objectif formatif.

Il reste à rapprocher la notion d'approche formative des techniques de correction des productions écrites; pour cela, il faut examiner l'exercice de production écrite dans le cadre qui est le sien, celui des stratégies cognitives. 


\subsubsection{La production écrite, une activité d'exercice et d'apprentissage de stratégies cognitives}

L'acte d'écriture fait appel de façon concomitante à un grand nombre de connaissances et d'habiletés. Nombre de ces habiletés sont de l'ordre des stratégies cognitives. Il ne saurait évidemment être question de mettre au clair dans ces pages une théorie exhaustive de l'acte d'écriture en référence aux stratégies cognitives et métacognitives, autant en raison de l'ampleur du propos que de l'état incomplet des connaissances en ce domaine.

Roy (1995) a produit un schéma en trois dimensions (voir figure 32) permettant de situer ces habiletés que sont les stratégies cognitives et métagognitives en regard des habiletés plus simples que sont les concepts et les règles. La figure 32 permet de voir que les stratégies cognitives et métacognitives sont des habiletés de soutien. Elles soutiendront donc dans l'exercice d'écriture l'usage des concepts et des règles connus, autant que l'effort créatif de l'ordre de la résolution de problème que l'exercice d'écriture commande à l'égard des éléments originaux du texte à produire. C'est là, en bref, les données sommaires de l'interprétation de l'exercice d'écriture en termes d'habiletés cognitives.

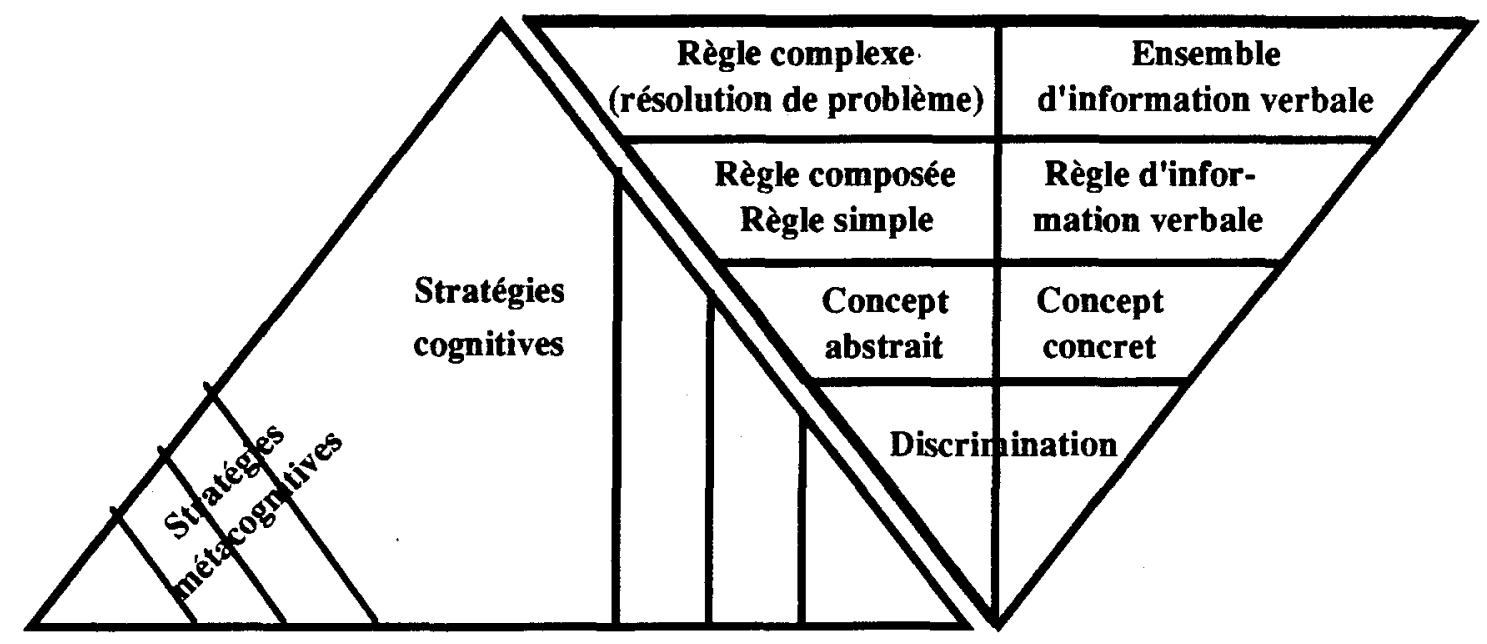

Figure 32: Schéma trois dimensions des habiletés cognitives (Roy, 1995). 
Or, comme le souligne Tardif (1993), les maîtres doivent exercer des interventions d'enseignement par rapport aux stratégies cognitives, et même métacognitives. La correction des productions écrites s'inscrit dans cet ordre d'intervention, et évidemment la correction à l'aide d'une grille. L'expérimentation relatée ci-après permet de voir comment pratiquement la grille de haut niveau qui a été présentée dans ces pages peut être adaptée pour convenir à des apprenants moins avancés et dans quel cadre et avec quelles préoccupations d'ordre formatif, il convient d'intégrer une grille de correction aux efforts d'apprentissages de l'écriture.

Dans le cadre d'une étude de la nature d'une recherche-milieu présentée au $17^{\mathrm{e}}$ congrès de L'AQEFLS (mars 1996), il a été fait application des propositions de cette recherche. Les chercheuses Simard et Gauthier (1996), toutes deux enseignantes à l'époque à l'École Selwyn House, ont expérimenté au cours de l'année scolaire 95-96 la grille de correction et son cadre d'utilisation, celui d'une approche formative.

Des centaines de copies de 19 étudiants d'une classe de cinquième année du primaire, immersion française, ont été corrigées à l'aide de la grille. Évidemment des choix de sous-catégories ont dû se faire étant donné le niveau et la clientèle cible. Un total de 30 sous-catégories ont été retenues, eu égard au niveau des élèves. L'enseignante était bien consciente qu'en formatif il ne convient pas de travailler avec toutes les sous-catégories de la grille, qu'il vaut mieux répondre aux besoins et adapter le contenu de la grille au niveau des étudiants.

C'est dans une optique respectueuse du cheminement des apprenants que s'est faite l'expérimentation. La correction qui a été réalisée poursuivait comme objectif de faire acquérir des stratégies de correction aux apprenants et ce, dans le but de les rendre plus 
autonomes face à leur démarche d'amélioration et d'apprentissage de l'écriture. Les buts de la correction étaient aussi sommatifs, car en bout de ligne une note se devait d'être donnée. Mais en préparation au produit fini, l'enseignante corrigeait les copies dans un objectif formatif.

Les cotes attribuées aux fautes relevées dans les copies référaient le scripteur non pas à des descripteurs des erreurs, mais à des stratégies de correction, ce que laisse voir la figure 33 présentée à la page suivante. Ainsi, pour une faute de la sous-catégorie 110 d'orthographe d'usage, il est conseillé à l'élève d'utiliser son dictionnaire; pour une faute de la sous-catégorie 210 de morphologie du nom, il est conseillé à l'élève de s'interroger sur le genre et le nombre du mot qu'il utilise. Par l'indication que lui transmettait la cote, le scripteur se référait à la grille et il savait quelle stratégie employer pour corriger son erreur.

Une autre stratégie, facilitant la correction des productions écrites des élèves de cinquième année du primaire, a été celle de remettre à chaque apprenant un modèle réduit et cartonné de la grille Mes outils de correction. Leur texte en main, corrigé par l'enseignante qui avait coté les fautes, les élèves se dirigeaient vers la salle d'ordinateurs pour y rapporter des corrections à leur texte. Leur grille cartonnée sous les yeux, ils pouvaient rapidement identifier la stratégie utile, la piste de correction leur permettant de s'interroger et de rectifier l'erreur commise.

Les retombées de cette expérience ont été d'aider les apprenants à apprendre certaines notions et à améliorer leur niveau d'écriture en français. Toutefois, ces améliorations n'ont pas pu être systématiquement mesurées. Mais une chose est certaine: les apprenants ont développé une plus grande autonomie face à leur tâche de correction. L'enseignante a 


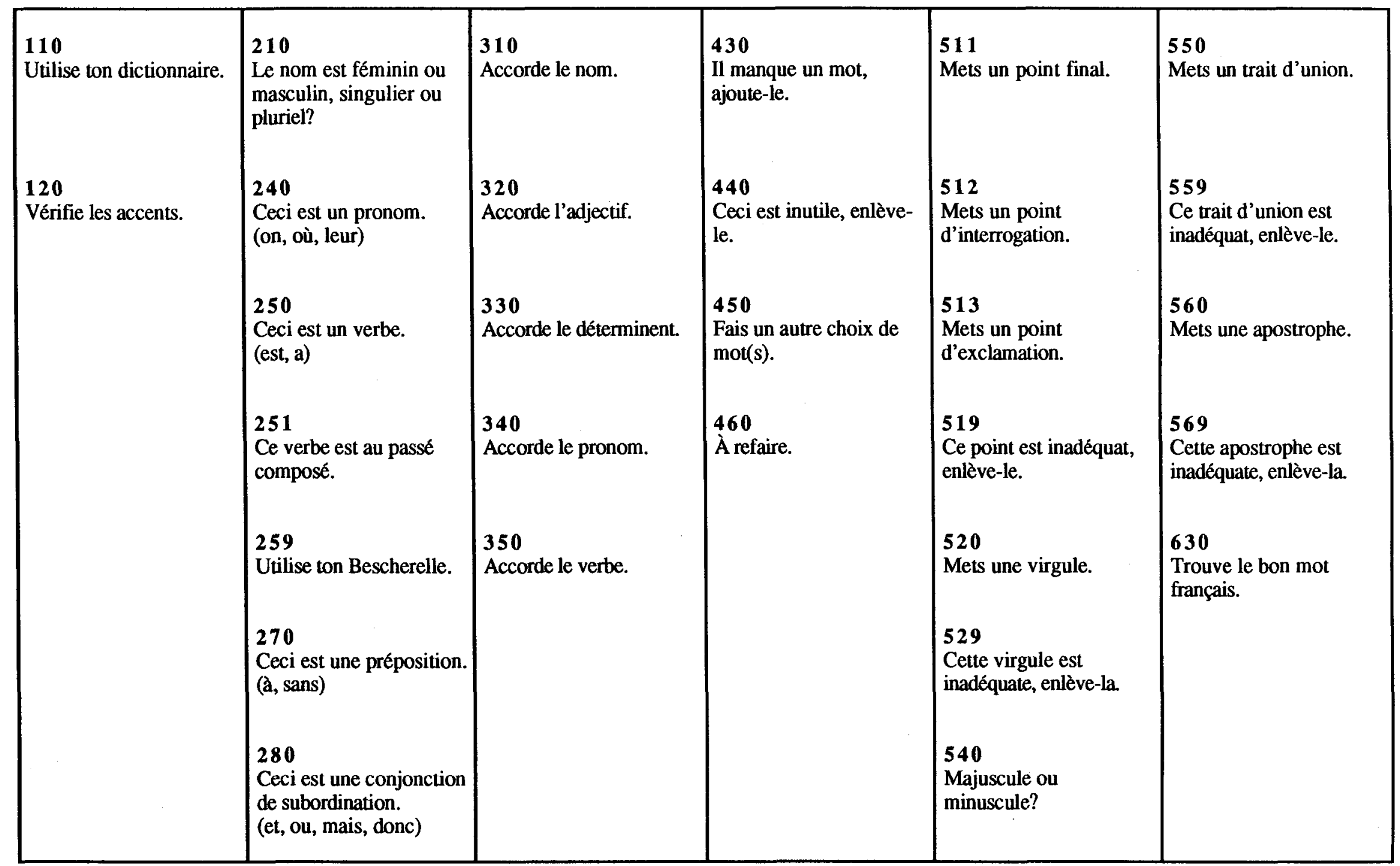

Figure 33: Mes outils de correction (Simard et Gauthier, 1996). 
d'ailleurs remarqué une très grande diminution des fréquences d'aide individualisée à son bureau. Les apprenants avaient, semble-t-il, les outils nécessaires pour faire seuls leur correction et pour trouver seuls les réponses à leurs questions. À la longue, ils étaient donc devenus plus habiles et surtout plus autonomes face à l'activité de correction de leurs productions écrites.

L'usage d'une grille permet de faire une correction utile, car elle permet, par sa précision diagnostique, d'aider le scripteur dans sa démarche d'écriture en lui proposant des pistes, des stratégies de correction. Au fil des interventions, l'apprenant améliore et augmente ses stratégies de correction. Vue de cette façon, la grille sera davantage considérée comme un guide, comme un outil précis permettant au maître de diagnostiquer les difficultés et permettant à l'élève de situer ses erreurs, de les comprendre, pour ensuite mieux les corriger et les intégrer. C'est ainsi que les cotes de la grille, qui réfêrent à des sous-catégories, permettent et au maître et à l'apprenant de cibler les efforts. Relever des erreurs peut paraître un acte négatif, mais en utilisant la grille afin de guider la correction, il est possible d'agir non pas pour sanctionner, mais bien pour provoquer correction et apprentissage. L'acte de corriger et de coter les fautes pourra alors être vu comme menant à des apprentissages. Un certain nombre de principes de correction formative gagnent à être respectés afin d'assurer toute sa qualité à l'activité de correction. Ce sont ces principes que présentent et commentent les dernières pages de ce chapitre.

\subsection{PRINCIPES DE CORRECTION FORMATIVE DE LA PRODUCTION ÉCRITE}

Viser à une correction efficace ou viser à une correction qui veut mener à des apprentissages nécessitera le respect de certaines conditions. Une de ces conditions pose 
que le correcteur agisse comme formateur et soit pénétré d'une approche formative. Lors d'expérimentations auprès d'étudiants universitaires, la correction des dissertations et des comptes rendus de lecture se faisait dans un cadre formatif. C'est ainsi qu'il a été possible de faire ressortir plusieurs principes de correction formative et ce, afin de réaliser une évaluation qui permette de mener les élèves à faire des apprentissages. Pour l'essentiel, ces principes sont des suggestions issues, une fois de plus, de la pratique. La figure 34 présente ces principes; ils seront par la suite commentés, puis discutés.

1 - Voir à intégrer la correction à la démarche d'apprentissage.

2 - Considérer le processus d'apprentissage de l'écriture comme une démarche progressive et continue.

3 - Éviter de corriger pour corriger, mais bien plutôt n'identifier que les fautes relevant du niveau et des capacités d'apprentissage du scripteur.

4 - Souligner de remarques positives les passages de qualité.

5 - S'arrêter aux difficultés majeures du groupe ou de l'apprenant et proposer des exercices correctifs se rapportant à ces difficultés.

6- Donner un suivi à sa correction, c'est-à-dire orienter ses activités d'enseignement selon les difficultés perçues.

7 - Amener l'apprenant à comparer ses performances avec ses propres performances antérieures, plutôt qu'avec celles du groupe.

8 - Référer l'apprenant à un portrait diagnostique global de ses difficultés.

9 - Toujours porter une attention particulière à la copie de l'élève et lui transmettre l'évaluation de sa performance dans de brefs délais.

Figure 34: Principes de correction formative.

Ces principes méritent qu'une attention particulière leur soit portée. D'abord celui qui vise à intégrer la correction à la démarche d'apprentissage (principe 1), donc de considérer l'élève comme un sujet d'apprentissage. Si la correction est faite vers cette optique, comme une démarche menant à l'apprentissage, l'apprenant la verra davantage comme une activité aidante qui lui permettra d'apprendre et non d'être sanctionné. Le maître gagnera 
aussi à considérer le processus d'apprentissage de l'écriture comme une démarche progressive et continue (principe 2). À titre d'exemple, il est difficile de vouloir faire acquérir des notions de style si le scripteur ne possède pas celles relatives à la structuration de la phrase. L'apprentissage de l'écriture se construit de plusieurs habiletés et règles qui, progressivement, en viennent à former un ensemble intégré. Ainsi, en correction, il vaudra mieux n'identifier que les fautes relevant du niveau et des capacités mêmes d'apprentissage du scripteur (principe 3). Le maître devra ainsi savoir où se situe le niveau de son apprenant et ne lui indiquer que les annotations qui s'imposent en regard du niveau de progression atteint.

La tentation est grande, surtout causée par la menace des programmes, de faire une correction selon le niveau académique donné. Une erreur à ne pas commettre est celle, avec ou sans recours à une grille, de corriger pour corriger, en cherchant à relever toutes les fautes, à la limite en cherchant à faire uniquement la preuve de l'acuité de son oeil de correcteur, sans souci des besoins des apprenants scripteurs. Corriger avec des exigences trop élevées n'aura pour effet que de décourager le scripteur, peut-être même de bloquer ses apprentissages. Il vaut mieux lui indiquer les fautes qu'il sera capable de comprendre et de corriger. Les erreurs d'un niveau plus élevé pourront être oubliées temporairement et n'être indiquées que lorsque le scripteur sera apte à les maîtriser. Certains pourraient trouver ce procédé malhonnête: ce serait ne pas comprendre le respect qu'exige un cheminement d'apprentissage, lequel ne peut être que progressif et continu. Ce cheminement pourrait être compromis par le manque de discernement. Évidemment, il devra s'établir une certaine complicité entre correcteur et scripteur, le second étant vaguement conscient que la correction s'adresse à ses besoins. La grille se présentera plus concise, ciblant les éléments sur lesquels maître et apprenants choisissent et conviennent de faire porter leurs efforts. 
Il a été possible de constater, lors des expérimentations de correction auprès d'étudiants universitaires, qu'à l'intérieur d'une seule session il fallait, au gré des apprentissages des étudiants, modifier aux cinq semaines l'entente sur le niveau de correction à assurer. D'ailleurs, une autre constatation qui a été faite est celle des effets de cheminement: c'est ainsi qu'un étudiant accédant à un niveau supérieur de maîtrise pourra avoir la surprise de faire autant de fautes, de commettre autant d'erreurs qu'à l'étape précédente de son apprentissage. Le correcteur devra être là pour faire prendre conscience à l'apprenant que son accession à un niveau supérieur d'habiletés le met devant des exigences d'écriture plus grandes et multiplie les possibilités d'erreur. Il en est ainsi du scripteur qui aborde la phrase longue avec ses difficultés de structure et ses exigences de ponctuation.

Faire une correction dans un cadre formatif suppose que des actions soient concrètement posées par le maître. D'abord le maître gagnera à transmettre à son apprenant des remarques positives (principe 4) et ce, pour la présence de passages de qualité dans un texte et pour l'application de règles maintenant maitrisées. Cela aura pour effet d'encourager l'apprenant et de lui donner confiance en la poursuite de son apprentissage de l'écriture.

Une autre action concrète et formative sera celle de remettre à son apprenant, ou à son groupe en général, des exercices correctifs se rapportant aux difficultés perçues (principe 5). L'idéal serait que le maître ait sous la main des exercices correctifs pour chacune des catégories et même des sous-catégories de la grille. Ces exercices agiront à titre de révision et permettront à l'apprenant de revoir certaines notions, de les appliquer et de les transposer dans ses activités d'écriture. Des exercices de ce type sont présentés en annexe (voir annexe 4). Les étudiants de niveau universitaire qui ont participé aux expérimentations éprouvaient principalement des difficultés pour les règles relatives aux sous-catégories Référent et Mot(s) mal choisi(s) $(420,450)$ de la catégorie Structure de la phrase, aux 
sous-catégories d'usage de la virgule $(521,522,523$ et 524$)$ de la catégorie Signes de ponctuation et conventions et finalement aux sous-catégories ( 721,722 et 723$)$ de la catégorie Stylistique. Ce sont donc ces exercices correctifs qui ont été construits et qui ont aidé les étudiants aux prises avec des difficultés pour ces notions fort particulières.

Un autre principe suggère de donner un suivi à sa correction, c'est-à-dire d'orienter ses activités d'enseignement selon les difficultés perçues (principe 6). Le maître gagnera à enseigner des règles non maîtrisées par l'ensemble du groupe; ces règles qui auront été déterminées par la correction qui aura été faite à l'aide de la grille et des portraits diagnostiques qui en auront résultés. Cette technique particulière que permet l'utilisation d'une grille de correction est celle, pour bien cibler la nature des difficultés de groupe, d'avoir recours à un logiciel de traitement des données qui permet rapidement d'obtenir un portrait diagnostique global des difficultés d'ensemble. C'est le logiciel File Force de Macintosh qui a été utilisé lors des expérimentations. Tout autre logiciel de traitement des données pourra faire adéquatement le travail, par exemple pour le primaire: Excel. Les cotes entrées à l'ordinateur étaient transmises à un traitement informatique qui occasionnait par la suite un portrait diagnostique pour chacun des groupes (voir annexe 5). Le maître était donc en mesure de savoir quelles difficultés éprouvaient son groupe. De ce fait, le maître pouvait savoir où et comment orienter ses activités d'enseignement.

Le maître agira dans ses enseignements pour le groupe, mais devra aussi agir pour chacun de ses apprenants et ce, en fonction du niveau de performance individuelle. Il importera donc d'amener l'apprenant à comparer ses performances avec ses performances antérieures et non pas avec celles du groupe (principe 7). Le maître devra informer sa classe que les niveaux de maîtrise de l'écriture sont différents pour chacun. De ce fait, étant donné que les difficultés ne sont pas les mêmes pour tous, il serait souhaitable de 
transmettre à l'étudiant un portrait diagnostique, mais cette fois-ci individuel (principe 8). Ce portrait a pour but d'aider à la prise de conscience et à la prise en charge par les apprenants de leur cheminement. Quelques-uns de ces documents ont aussi été fournis en cours de sessions aux étudiants universitaires; un exemple de portrait individuel est présenté en annexe (voir annexe 6). Cette pratique, de transmettre un portrait individuel, a pour principal objectif que le scripteur mobilise ses efforts aux endroits où il commet le plus d'erreurs et qu'il puisse, de ce fait, constater sa progression relativement à sa propre performance.

Advenant le cas où les propositions faites sur l'évaluation formative ne convenaient pas au correcteur, le seul et principal principe qu'il faudrait observer en correction des productions écrites serait le dernier principe (principe 9), soit de toujours porter une attention particulière à la copie de son élève et de lui transmettre l'évaluation de sa performance dans de brefs délais. Ce principe pourra, pour certains, apparaître simplet. Ce serait ne pas comprendre l'effet qu'a la correction immédiate sur l'élève. L'action posée qui est celle de s'arrêter à un élève et à sa copie aura en soi un effet formateur sur l'élève, si cette correction lui est transmise dans de courts délais.

Le troisième chapitre a fourni la base d'une correction formative issue d'expériences de correction. Pour l'essentiel, les connaissances produites à l'intérieur de ce chapitre ont été l'évaluation formative rapprochée aux théories cognitives de Gagné. À partir des données de cette théorie, il a été possible de rattacher l'évaluation formative à la grille de correction ayant fait l'objet du deuxième chapitre. Par la suite, quelques principes formatifs ont été émis et ce, dans le but d'illustrer les rapprochements entre évaluation formative et la correction. L'activité d'évaluer pour former a aussi fait naître des perspectives d'intervention. Elles ont été pour l'essentiel l'élaboration de matériel correctif et la transmission 
de portraits diagnostiques aux élèves. Ces pratiques ont pour but, d'une part, de faire prendre conscience à l'élève de ses difficultés majeures et, d'autre part, de lui donner les moyens pour les contrer.

La pratique de correction proposée, soit celle de corriger avec une grille diagnostique, puis d'observer de sains principes didactiques et formatifs pourra rebuter. Toutefois, le maître qui en fera l'effort découvrira tout l'intérêt du respect de cheminement de son apprenant en apprentissage et aussi tout l'intérêt, lors de l'utilisation de la grille, d'établir un diagnostic permettant de comprendre derrière les erreurs les difficultés de ses apprenants. Le maître se retrouvera à même de mieux guider ses scripteurs comme de mieux orienter ses interventions d'enseignement. 
CONCLUSION 
Cette recherche-développement a permis d'accorder une attention renouvelée à la correction des productions écrites en français. Elle a proposé un ensemble permettant de faire une correction de haute qualité. Essentiellement, il fut question de proposer, d'une part, une grille diagnostique renouvelée et, d'autre part, des principes de correction formative venant guider la correction des productions écrites.

Malgré les atouts didactiques novateurs de la grille, il est à prévoir que son utilisation et son application ne s'avéreront pas une solution évidente pour tous les maitres. Certains trouveront la grille trop complexe, trop lourde, trop détaillée. D'autres ne verront pas toute la portée heuristique à appliquer de sains principes didactiques et formatifs. Par ailleurs, proposer une grille nouvelle d'utilisation formative en rebutera sûrement quelques-uns conquis aux méthodes traditionnelles de correction. Ces méthodes, peut-être simplistes, escamotent parfois les conceptions modernes de l'apprentissage. Aux yeux de plusieurs, ces méthodes contiennent de nombreux pièges auxquels n'échappent pas les exercices de productions écrites et leurs corrections.

Un de ces pièges se situe dans la correction même des productions écrites. Cet exercice traditionnel consiste souvent à réaliser des inventaires de fautes. Cette façon de corriger les textes oriente davantage le maître à condamner ses apprenants, plutôt qu'à leur venir en aide de façon constructive.

Il faudrait considérer l'activité de correction autrement, c'est-à-dire ne plus surcharger les copies de rouge: cela a pour effet de sanctionner et de brimer les efforts d'écriture des apprenants; ce qui engendre une source additionnelle de démotivation. Un change- 
ment de perception se doit d'être fait: la correction devrait plutôt être vue comme une activité aidante, permettant un dialogue entre maître et apprenants et ce, dans le but de transmettre de façon progressive des stratégies de correction visant l'apprentissage et la maîtrise de l'écriture en français. Évidemment cet apprentissage se fera sur une longue période et engagera tant le maître que l'apprenant à s'entendre sur certains aspects.

À cet effet, maître et apprenant pourraient convenir que le premier n'exige pas de l'autre une maitrise totale et parfaite de la langue. II serait possible, par exemple, de poser comme principe qu'en deçà de cinq erreurs, pour un texte de 300 mots (ou toute autre norme conforme au niveau de l'apprenant), la performance pourrait entraîner une note parfaite au même titre qu'une copie sans faute.

L'activité d'écriture est humaine, l'activité de correction qui en résulte se devrait aussi d'être humaine. Le maître doit échapper au piège de comptabiliser les fautes. Il faut créer un nouveau mode d'évaluation qui vise, plutôt qu'une condamnation, une maîtrise et une compréhension profonde de l'écriture.

Un autre piège observé concerne l'implantation, dans les classes de français, d'une certaine mode voulant retarder l'application des règles grammaticales ou orthographiques dans la production d'un texte écrit, à l'état du brouillon. Pour des raisons obscures, l'application du programme de 1979 a entraîné les maîtres à proposer cette règle de fonctionnement, règle pernicieuse dans ses effets. Cette façon de faire s'expliquerait par le fait que certains maitres croient que réfléchir aux notions de langue aurait pour effet de nuire à l'exercice de la pensée. Or, ce qui est omis, c'est qu'écrire repose avant tout sur une mâtrise préalable de la langue. $D$ 'autres adopteront cette pratique afin de retarder les exercices d'objectivation permettant -peut-être- aux apprenants de prendre conscience de leurs difficultés d'écriture. 
Ce fut et c'est toujours une erreur de penser qu'il y a avantage à se corriger plus tard et à revenir sur son texte. Il faudrait plutôt créer des automatismes dans l'application des règles de la grammaire et amener les apprenants à écrire tout en s'arrêtant autant sur les phénomènes de la langue que sur ceux de la pensée.

Les maîtres, préoccupés par la qualité des apprentissages de leurs élèves, gagneront à contrer ces pièges. D'ailleurs, la grille qui vient d'être proposée dans cette étude sera très utile à cet égard. Il reste à espérer que les enseignants arrivent principalement à créer un nouveau mode d'évaluation des productions écrites plus respectueux, puis à trouver les moyens permettant de développer des automatismes d'écriture dans l'application de règles. Toutefois, pour atteindre ces objectifs, il faudra accepter de corriger, donc de faire produire du texte, régulièrement et surtout souvent. Le travail de correction qui en résulte est inévitable, au sens où ne peuvent être contournées les heures d'un travail attentif à corriger des copies. Il est vrai que les circonstances actuelles dans lesquelles le calcul des heures régulières de travail et l'augmentation des tâches sont préoccupantes, engagent les maîtres à ne pas avoir le temps nécessaire pour la correction, donc pas plus que celui pour faire produire du texte écrit.

Si les maîtres se sentent coincés dans le système, il faudrait au moins qu'ils réussissent à se donner des moyens pour faire écrire leurs apprenants en sacrifiant même, à la limite, la correction. Il faut faire écrire les apprenants si l'on veut qu'ils apprennent à écrire et ce, même si les conditions difficiles des professeurs de français ne permettent pas d'en faire une correction approfondie. Or, pour le lecteur qui s'est acharné en vain à faire la lecture de cette étude, il ne faudrait pas croire que les grilles de correction n'ont plus leur place. 
Tout au contraire, le maître qui se donnera la peine et qui prendra le temps, d'une part, de corriger avec une grille complète, précise et diagnostique et, d'autre part, d'observer de sains principes de correction formative verra tous les effets positifs qu'a sa pratique sur ses élèves. Le maître pourra alors se réjouir de mener avec ses apprenants une activité de correction formative des productions écrites qui permette à la fois de diagnostiquer les difficultés de parcours et de respecter le cheminement de chaque apprenant inscrit dans un processus d'apprentissage de l'écriture. 


\section{RÉFÉRENCES \\ BIBLIOGRAPHIQUES}


ALBALAT, Antoine (1992). L'art d'écrire: enseigné en vingt leçons. Paris: A. Colin.

BEAULIEU, Georges, BOURGEAU, Jean-Pierre et PAQUIN, Michel (RIEFEC) (1977). Les erreurs les plus fréquentes en français écrit au collégial: les participes passés et les formes adjectivales: description des comportements déviants, matériel didactique correctif. Montréal: Collège Bois-de-Boulogne; Ste-Thérèse: Collège Lionel-Groulx.

BEAULIEU, Georges, BOURGEAU, Jean-Pierre et PAQUIN, Michel (RIEFEC) (1978). Consolidation des acquis en français écrit au collégial. Québec: Direction générale de l'enseignement collégial.

BIBEAU, Gilles (1975). Enquête sur le français écrit dans les cégeps. Montréal: Cégep de Maisonneuve.

BOYER, Jean-Yves, ROY, Raymond Claude et ROY, Gérard-Raymond (1991). «Les enseignants apprennent-ils à écrire à l'Université?», Revue Québec français, Hiver, $\mathrm{n}^{\circ} 80, \mathrm{pp} .41-43$.

BROUSSEAU, André, GARET, Nicole, JEAN, Lionel et LECLERC, Jacques (1991). Le français pour l'essentiel. Laval (Québec): Mondia Éditeurs inc.

BUREAU, Conrad (1985). Le français écrit au secondaire: une enquête et ses implications pédagogiques. Québec: Le conseil.

DE CORTE, E., GEERLIGS, C.T., LAGERWEIJ, N.A.J., PETERS, J.J.et VANDERBERGHE, R. (1979). Les fondements de l'action didactique. Bruxelles: Éditions A. De Boeck.

DES LAURIERS, Josée et MAJOR, Pierre (1992). Cent fautes ou sans faute?? Montréal: La Commission scolaire Sainte-Croix, $2^{e}$ édition.

FLAUX, Nelly (1993). La grammaire. Paris: Presses Universitaires de France.

GAGNÉ, France (1986). L'évaluation formative, la qualité du français écrit et l'attitude des étudiants a l'égard de l'évaluation, Mémoire de maîtrise. Rimouski: Université du Québec à Rimouski.

GAGNÉ, G., R. LAZURE, L. SPRENGER-CHAROLLES et ROPÉ, F. (1989). Recherches en didactique et acquisition du français langue maternelle. Bruxelles: De Boeck-Wesmael, tome 1. 
GAGNÉ, Gilles (1995). «La langue de Molière dans la francophonie», Journal La Presse, Édition du 3 novembre, Montréal.

GAGNÉ, Robert Mills (1976). Les principes fondamentaux de l'apprentissage. Montréal: Éditions HRW.

GAGNON, Pierre (1993). «Un instrument diagnostique en écriture», Revue Québec français, Automne, $\mathrm{n}^{\circ} 91, \mathrm{pp} .28-30$.

GARNEAU, Jacques (1993). Le test de français écrit des collèges et des universités. Ville Saint-Laurent (Québec): Éditions Trécarré.

GOOSSE, André (1986). Le bon usage. Gembloux (Paris): Duculot, Édition du renouveau pédagogique, $12^{\mathrm{e}}$ édition.

GREVISSE, Maurice (1980). Le bon usage. Gembloux (Paris): Duculot, Édition du renouveau pédagogique, $12^{e}$ édition.

JEAN, Lionel, LECLERC, Jacques et RACINE, Claude (1988). La grille de correction du SA.L.F.: mode d'emploi. Montréal: Collège de Bois-de-Boulogne.

JEAN, Sonya (1992). Projet de recherche sur la maîtrise d'écriture des formes verbales par des enfants de cinquième année du primaire. Chicoutimi: Université du Québec à Chicoutimi.

LÉPINE, François (1995). «Bilan des tests de français à l'admission aux universités québécoises (1987-1994)», Revue des sciences de l'éducation, Numéro thématique, vol. XXI, $\mathrm{n}^{\circ} 1$, pp.17-35.

MAROUZEAU, Jules (1963), Précis de stylistique française. Paris: Masson, $5^{e}$ édition.

MARTIN, André, HAMERS, Josiane et GODIN, Jean (1978). L'évaluation de la compétence linguistique et du vocabulaire actif des étudiants de première session au niveau collégial: rapport de recherche. Québec: Direction générale de l'Enseignement collégial.

MOTTOULLE, Marie-Jeanne (1957). Pratique du style. Paris: Éditions scientifiques et littéraires, $3^{e}$ édition.

ROBERGE, Albert (1984). Étude comparative sur l'orthographe d'élèves québécois. Québec: Le conseil.

ROBERGE, Julie (1993). «Une expérience d'évaluation formative. La correction sur...cassette», Revue Québec français, Printemps, $n^{\circ} 89$, pp.50-51. 
ROY, Gérard-Raymond et LAFONTAINE, Louise (1992). Étude de la maîtrise du français écrit à l'Université. Sherbrooke: Éditions du CRP.

ROY, Raymond Claude (1995). Concrétisation de l'approche cognitive d'enseignementapprentissage par découverte de R.M. Gagné en enseignement de la grammaire française (figures). Chicoutimi: Groupe de recherche en grammaire et didactique du français, Département des sciences de l'Éducation, Université du Québec à Chicoutimi.

SCALLON, Gérard (1988). L'évaluation formative des apprentissages. Québec: Les Presses de l'Université Laval.

SIMARD, Nathalie et GAUTHIER, France (1996). «Une grille et des stratégies de correction basées sur des principes formatifs: un mélange parfait pour corriger les lacunes d'écriture en français», Revue de l'AQEFLS, octobre, nos 1-2, vol. 18.

TARDIF, Jacques (1993). «Pour un enseignement de plus en plus stratégique», Revue Québec Français, printemps, n 89 , pp.35-39.

THIMONNIER, René (1974). Code orthographique et grammatical. Paris: A. Hatier.

TROESTLER, Pierre-Paul (1978). Orthographe professionnelk- Essai de typologie des fautes. Jonquière: Collège de Jonquière, Service de recherche et expérimentation. 


\section{ANNEXE I}

Guide de correction

référentielle et interactive 


\section{UNIVERSITÉ DU QUÉBEC À CHICOUTIMI}

Maîtrise en Éducation

\section{Guide

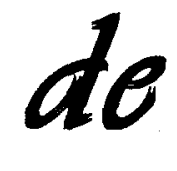 \\ correction référentielle et interactive}

CONÇU PAR

Nathalie Simard

SOUS LA DIRECTION DE Raymond Claude Roy

Version finale (mai 1996) 


\section{UNIVERSITÉ DU QUÉBEC À CHICOUTIMI}

Maîtrise en Éducation

\section{Guide de correction réferentielle et interactive \\ CONÇU PAR \\ Nathalie Simard}

SOUS LA DIRECTION DE

Raymond Claude Roy

Version finale (mai 1996) 


\section{À NOTER}

(1) Ce guide descriptif a été élaboré à partir, et entre autres, du travail de recherche sur la virgule de Madame Sonya Jean, du guide de correction de Madame France Gauthier, ainsi que de la recherche intitulée: La compétence en français écrit des étudiants en éducation du réseau de l'Université du Québec (1991), coordonnée par Messieurs Jean-Yves Boyer, Raymond Claude Roy et Jean A. Roy. Ce guide présente toutefois des développements plus récents.

(2) Pour les approfondissements théoriques et les détails, le correcteur est invité à consulter le Mémoire de maîtrise de Nathalie Simard (1996) intitulé: Proposition de grille et de principes formatifs pour la correction des productions écrites, d'où ce guide et ses propos ont pris naissance. 


\section{TABLE DES MATIÈRES}

1 - Table des matières

2 - Règles d'utilisation de la grille

3 - Principes de correction formative ……………......................................................................... 6

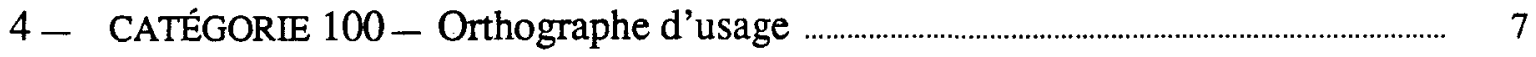

5 - CATÉGORIE 200 - Morphologie …................................................................. 13

6 - CATÉGORIE 300 - Accord ……................................................................................. 25

7 - CATÉGORIE 400 - Structure de la phrase ……................................................................ 39

8 - CATÉGORIE 500 - Signes de ponctuation et conventions ….......................................... 47

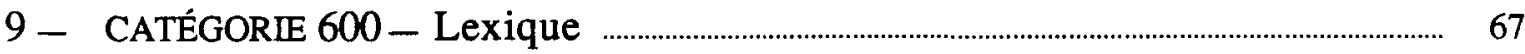

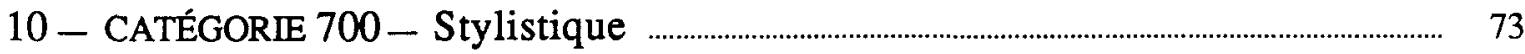

11 - CATÉGORIE 800 - Qualité textuelle ......................................................................... 77

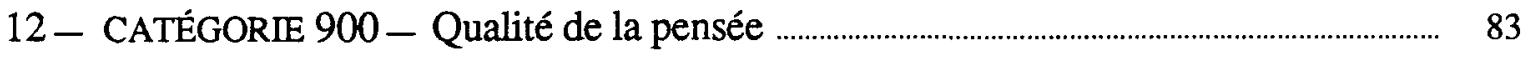

13 - Grille de correction ......................................................................................................... 


\section{RÈGLES D'UTILISATION DE LA GRILLE}

1- La cote ou la catégorie de classification de la faute est déterminée en fonction de la bonne réponse, et non selon l'erreur commise. Dans l'exemple suivant:

$$
\text { Viens-tu ce soir, }
$$

la faute sera classée dans la sous-catégorie Point d'interrogation (512) et non à la sous-catégorie Point (510).

2 - Le classement d'une faute devrait se faire du point de vue de l'apprentissage à maîtriser. Dans: «cette autobus», la faute relève de la morphologie du nom (210), et non de la sous-catégorie de l'adjectif démonstratif (330).

3- Dans le cas de deux virgules à utiliser en complémentarité, il ne sera enregistré qu'une seule erreur. Tel sera le cas lors de l'utilisation d'une virgule explicative.

4- Lorsqu'il y a plus d'une faute dans un même mot, toutes les fautes sont relevées à condition qu'elles soient de catégories différentes, comme le montre bien cet exemple:

il fésais (110), (250)

5- Lorsque la même faute revient plusieurs fois, il est suggéré de mettre la cote entre parenthèses ( ). 
6- Le correcteur doit se contenter d'identifier les erreurs présentes dans le texte et éviter de refaire complètement l'écriture (sauf, à l'occasion, pour montrer au scripteur ce qui aurait pu être une formulation intéressante).

7- Des commentaires positifs gagneraient à être ajoutés en marge de la copie pour souligner les forces de l'écriture et du texte. 


\section{PRINCIPES DE CORRECTION FORMATIVE}

1- Voir à intégrer la correction à la démarche d'apprentissage.

2- Considérer le processus d'apprentissage de l'écriture comme une démarche progressive et continue.

3- Éviter de corriger pour corriger, mais bien plutôt n'identifier que les fautes relevant du niveau et des capacités d'apprentissage du scripteur.

4- Souligner de remarques positives les passages de qualité.

5- S'arrêter aux difficultés majeures du groupe ou de l'apprenant et proposer des exercices correctifs se rapportant à ces difficultés.

6- Donner un suivi à sa correction, c'est-à-dire orienter ses activités d'enseignement selon les difficultés perçues.

7- Amener l'apprenant à comparer ses performances avec ses propres performances antérieures, plutôt qu'avec celles du groupe.

8- Référer l'apprenant à un portrait diagnostique global de ses difficultés.

9- Toujours porter une attention particulière à la copie de l'élève et lui transmettre l'évaluation de sa performance dans de brefs délais. 


\section{ORTHOGRAPHE D'USAGE}

Grevisse définit l'orthographe comme étant «la manière d'écrire correctement les mots d'une langue». «L'orthographe d'usage a pour objet les mots pris en eux-mêmes, tels que les donnent les dictionnaires, sans égard à leur rôle dans la phrase (Grevisse et Goosse, 1986, pp. 99-100).» Plus précisément, appartiendront à la catégorie Orthographe d'usage la ou les parties du mot qui ne présentent pas de dimensions grammaticales.

Ainsi, l'orthographe d'usage est traitée en quatre sous-catégories qui seront tout d'abord décrites, puis illustrées. Plusieurs exemples d'erreurs possibles seront fournis.

$$
\begin{aligned}
& 110 \text { - Cas général } \\
& 120 \text { - Signes auxiliaires } \\
& 130 \text { - Géminée } \\
& 199 \text { - Inclassable }
\end{aligned}
$$




\title{
ERREUR TYPE:
}

\author{
$\underline{\text { Saint - Sain }}$
}

Cette sous-catégorie fait référence aux fautes commises dans l'écriture d'un mot de vocabulaire qu'il est possible de trouver dans le dictionnaire et ne présentant pas, au total, de dimensions grammaticales. La sous-catégorie Cas général englobe toutes les fautes d'orthographe d'usage autres que celles relatives à l'emploi des signes auxiliaires et de la géminée.

\begin{tabular}{|cll|}
\hline & ERREUR & FORME CORRECTE \\
$1-\quad$ Shéma & Schéma \\
$2-$ & Temp & Temps \\
$3-$ & Exacte & Exact \\
$4-$ & Avoir recourt & Avoir recours \\
$5-$ Douçâtre & Douceâtre \\
$6-$ Deuille & Deuil \\
$7-$ Dicté & Dictée \\
$8-$ Voire & Voir \\
$9-$ Foi (de porc) & Foie \\
$10-$ Acceuil & Accueil \\
$11-$ Méfience & Méfiance \\
$12-$ Longeur & Longueur \\
\hline
\end{tabular}




\section{ERREUR TYPE:}

$$
\text { Concu - Conçu }
$$

Cette sous-catégorie relève des fautes d'accents (circonflexe, aigu, grave), du tréma, de la cédille, de la séparation des mots, de l'apostrophe et du trait d'union. Ces signes peuvent ou manquer ou être erronément présents dans un mot de vocabulaire. Il faut toutefois s'assurer que l'erreur de signe ne fait pas plutôt référence à la morphologie, ce qui serait le cas dans «ils demanderent» (250).

\section{ERREUR}

1 - Amere

2- Acquièrt

3- Premierement

4- Épitre

5 - Foret

6- Gout

7- Vetement

8- Nécéssaire

9- Quelques uns

10- Héroique

11 - Coincider

12- Celà

13 - C'est à dire

14- Presque île 1

15- Évènement
FORME CORRECTE

Amère

Acquiert

Premièrement

Épître (épistémologie)

Forêt

Goût

Vêtement

Nécessaire

Quelques-uns

Héroïque

Coïncider

Cela

C'est-à-dire

Presqu'île

Événement

1 Ce cas de non-emploi de l'apostrophe entre bel et bien dans cette sous-catégorie, car ce mot est un mot d'orthographe usuel du dictionnaire. Toutefois, des cas d'utilisation de l'apostrophe, tels que epresquil», «lorganisation», «jusquà», «dabord», «quà la base», etc. seront à classer dans la sous-catégorie 560, surnommée: Apostrophe d"élision (voir p.65). 


\title{
ERREUR TYPE:
}

\author{
Apeler - Appeler
}

«Il y a géminée lorsqu'il y a succession de deux consonnes identiques (Grevisse et Goosse, 1986, p.45).» Dans un mot de vocabulaire, il est possible qu'un scripteur ou omette la géminée (aporte), ou en introduise une à tort (proffesseur). Dans ce cas, où la géminée est inventée, l'erreur est normalement classée à la catégorie: Cas général. Toutefois, il est conseillé de considérer ces erreurs comme des erreurs de géminée et ce, pour la particularité de certaines règles communes.

\begin{tabular}{|c|c|c|}
\hline & ERREUR & FORME CORRECTE \\
\hline $1-$ & Jardinnier 1 & Jardinier \\
\hline $2-$ & Freinnage 1 & Freinage \\
\hline $3-$ & Milion & Million \\
\hline 4 & Barronne ${ }^{1}$ & Baronne \\
\hline $5-$ & Appercevoir ${ }^{1}$ & Apercevoir \\
\hline $6-$ & Actionaire & Actionnaire \\
\hline $7-$ & Collectioneur & Collectionneur \\
\hline $8-$ & Traditionel & Traditionnel \\
\hline $9-$ & Eneigé & Enneigé \\
\hline $10-$ & Horraire' & Horaire \\
\hline $11-$ & Innacceptable $\mathbf{1}$ & Inacceptable \\
\hline $12-$ & Prudement & Prudemment \\
\hline
\end{tabular}

1 Tous ces cas sont des emplois de géminée inventée; ils devraient se retrouver nécessairement dans la sous-catégorie 110 . Toutefois, pour les besoins du maître et pour ceux de son enseignement, il est suggéré de regrouper tous les cas d'emploi de la géminée, soit fautif ou soit absent, dans cette souscatégorie. 


\section{INCLASSABLE}

\section{9}

La catégorie 199 Inclassable permet de regrouper toutes les fautes n'appartenant ni à l'une ni à l'autre des sous-catégories contenues à l'intérieur de la catégorie Orthographe d'usage. 


\section{MORPHOLOGIE}

Cette catégorie réunit toutes les fautes qui relèvent des variations de formes que subit un mot eu égard aux paradigmes grammaticaux existants. Il importe de distinguer la faute de morphologie de la faute d'accord; la faute de morphologie résulte plutôt de la méconnaissance de l'orthographe d'une forme en regard de sa nature de mot. Connaître la morphologie d'un mot, c'est connaître les transformations grammaticales possibles du mot et choisir la graphie qui convient pour un emploi donné. La plupart des fautes présentant des caractéristiques d'homophonie appartiennent à cette catégorie.

La catégorie Morphologie se subdivise forcément en onze sous-catégories reprenant les parties du discours. Ces sous-catégories seront décrites et plusieurs exemples en seront fournis.

210 - Nom
$220-$ Adjectif qualificatif
230 - Article et adjectif déterminatif
240 - Pronom
250 - Verbe
260 - Adverbe
$270-$ Préposition
280 - Conjonction de subordination
290 - Conjonction de coordination
295 - Interjection
299 - Inclassable




\title{
ERREUR TYPE:
}

\author{
Une avion - Un avion
}

Cette sous-catégorie identifie des erreurs originant de confusions touchant au genre («une avion») ou au nombre des noms («des pneux»), ou encore de confusions touchant à la forme que déterminent le genre et le nombre du nom («une veufe»).

Les règles de majuscules touchant les noms propres entrent normalement dans cette souscatégorie étant donné qu'il ne s'agit pas là d'une règle de ponctuation, mais bien d'une règle rattachée à la reconnaissance du mot qu'est le nom propre. Toutefois, pour des raisons de simplification, ces fautes pourraient être classées en catégories 540: Majuscule, minuscule et capitale.

$\begin{array}{ll} & \text { ERREUR } \\ 1- & \text { Fait parti } \\ 2- & \text { Des pneux } \\ 3- & \text { Des hibous } \\ 4- & \text { Une veufe } \\ 5- & \text { Une exécuteuse } \\ 6- & \text { Une avion } 1 \\ 7- & \text { Cette autobus } \\ 8- & \text { Ma vacance } \\ 9- & \text { Mes haines }\end{array}$

FORME CORRECTE

Fait partie

Des pneus

Des hiboux

Une veuve

Une exécutrice

Un avion

Cet autobus

Mes vacances

Ma haine

1 Il serait possible de croire que le scripteur n'ait pas su accorder correctement le déterminant, mais au contraire, il ne connaît pas le paradigme de genre affectant le nom qu'il a utilisé. Cette faute est donc de morphologie du nom (210) et non d'accord de l'article et de l'adjectif déterminatif (330). 


\section{ADJECTIF QUALIFICATIF}

\section{ERREUR TYPE:}

Légerte-Légère

Pour la sous-catégorie Morphologie de l'adjectif, la faute résulte d'un manque de maîtrise des variations sémiologiques possibles.

\section{ERREUR}

1 - Ville public

2- Fille fiere (m: fier) ${ }^{1}$

3 - Chere amie (m: cher) 1

4 - Voix aigue (m: aigu) 1

5 - Fille naife

6 - Personne enchanteuse ${ }^{2}$

7 - Légerte ${ }^{2}$

8- Un beau hôpital

9- Des bels hommes

10- Les peuples hébreus

11 - Principes morals

12 - Règlements principals

13 - Les noms fixent

14 - Le peuple Québécois ${ }^{3}$

\section{FORME CORRECTE}

Ville publique

Fille fière

Chère amie

Voix aiguë

Fille naive

Personne enchanteresse

Légère

Un bel hôpital

De beaux hommes

Les peuples hébreux

Principes moraux

Règlements principaux

Les noms fixes

Le peuple québécois

1 Les cas 2-3-4 peuvent être classés en sous-catégorie: Signes auxiliaires (120); encore une fois cela dépend des besoins du professeur et surtout du niveau d'apprentissage de son groupe.

2 Certains des cas présentés pourraient être vus comme des barbarismes, car les emplois sont inventés. Le choix de classement se déterminera selon les besoins de l'enseignant et de l'enseignement à en faire.

3 Un enseignant pourra aussi choisir de classer cette faute à la sous-catégorie 540: Majuscule, minuscule et capitale. Toutefois, le scripteur pourra davantage corriger cette erreur s'il est conscient de la nature d'adjectif du mot. 


\title{
230 ARTICLE ET ADJECTIF DÉTERMINATIF
}

\section{ERREUR TYPE:}

\author{
Vingts - Vingt
}

Les principales erreurs de cette sous-catégorie originent d'un manque de maîtrise de la sémiologie, surtout des adjectifs possessifs et des adjectifs démonstratifs.

\begin{tabular}{|lll|}
\hline & ERREUR & FORME CORRECTE \\
$1-$ & Un sel 1 & \\
$2-$ & Il n'y a pas le pain1 & Du sel \\
$3-$ & Dès plus beaux vêtements & Il n'y a pas de pain \\
$4-$ & Se référer au précédente & Se référer à la prétédente \\
$5-$ & Vingts hommes & Vingt homme \\
$6-$ & C'est amis & Ses amis \\
$7-$ & Ça preuve & Sa preuve \\
$8-$ & T'ont service & Ton service \\
$9-$ & Sont vestiaire & Son vestiaire \\
$10-$ & Donne-moi se livre & Conne-moi ce livre \\
$11-$ & Ses maisons-là & Cet espace \\
$12-$ & Ce espace & Pour ce faire \\
$13-$ & Pour se faire & Quelles sont les raisons? \\
$14-$ & Qu'elles sont les raisons? & Quelles vies! \\
$15-$ & Qu'elles vies! & Chaque concept \\
$16-$ & Chaques concepts & Tout le monde \\
$17-$ & Tous le monde & Quelle que soit ta vie \\
$18-$ & Quelque soit ta vie & Quelques raisons \\
$19-$ & Quelles que raisons & \\
\hline
\end{tabular}

1 Les exemples 1 et 2 seront des erreurs commises généralement par des anglophones. Le scripteur possédant son français (langue maternelle) ne fera ordinairement pas ce type d'erreur. 


\section{ERREUR TYPE:}

Ont - On

Ces fautes originent de la méconnaissance sémiologique des pronoms.

\begin{tabular}{|cll|}
\hline & ERREUR & FORME CORRECTE \\
$1-$ & Chacun pour soit & Chacun pour soi \\
$2-$ & Il l'a seconde & Il la seconde \\
$3-$ & Quelle est jolie & Qu'elle est jolie \\
$4-$ & Il c'est blessé & Il s'est blessé \\
$5-$ & L'animal ce cache & L'animal se cache \\
$6-$ & Il ni trouve pas & Il n'y trouve pas \\
$7-$ & Il est possible dans voir & Il est possible d'en voir \\
$8-$ & Se n'est pas loyal & Ce n'est pas loyal \\
$9-$ & Ces juste de croire que & C'est juste de croire que \\
$10-$ & Sa suffit! & Ça suffit! \\
$11-$ & Quoique j'en pense & Quoi que j'en pense \\
$12-$ & La compagnie pour la quelle... & La compagnie pour laquelle... \\
$13-$ & De l'endroit ou vous êtes & De l'endroit où vous êtes \\
$14-$ & À qu'y parles-tu? & Á qui parles-tu? \\
$15-$ & Ont les respecte & On les respecte \\
& & \\
\hline
\end{tabular}




\title{
250
}

\section{VERBE}

\section{ERREUR TYPE:}

\author{
J'entend - J'entends \\ Il veut lavé - II veut laver
}

Les erreurs de cette soús`catégorie résultent le plus souvent d'une méconnaissance du système du verbe et de sa sémiologie.

\section{ERREUR}

$1-$ Il a lit

2- Il a réussit

3 - Les types vuent

4- Elles se sont introduient

5 - Il a permi

6- Il fesait ${ }^{1}$

7- Définire1

8- Je mangais 1

9- Ce fût agréable

10- II voirra les changements

11 - Il suivera la course

12 - Ais du courage

13- Je ne voie

14- Nous diront ${ }^{2}$

15- Il emploi

16- Manges ta soupe!

17- II à faim

18 - Ils on appris la leçon

19- Veuillez expliquez ce problème

\section{FORME CORRECTE}

Il a lu

Il a réussi

Les types vus

Elles se sont introduites

Il a permis

Il faisait

Définir

Je mangeais

Ce fut agréable

Il verra les changements

II suivra la course

Aie du courage

Je ne vois

Nous dirons

Il emploie

Mange ta soupe!

Il a faim

Ils ont appris la leçon

Veuillez expliquer ce problème

1 Ces cas peuvent aussi être considérés comme des cas référant à l'orthographe d'usage.

2 Ce cas représente une connaissance de morphologie de base. Quelqu'un qui connaît la morphologie ne pourra pas faire de faute d'accord. Si ce type d'erreur est fait, c'est en morphologie qu'il devra être classé. Toutefois, si le maître de niveau élémentaire décide que cette connaissance n'est pas encore acquise chez ses étudiants, il pourra la considérer comme une faute d'accord; une fois acquises et maîtrisées, des erreurs de ce type seront à classer en morphologie. 


\title{
ADVERBE
}

\section{ERREUR TYPE:}

\author{
$\underline{\text { Peut }- \text { Peu }}$
}

Les erreurs de forme touchant les adverbes entreront dans cette sous-catégorie, même si parfois l'erreur peut être vue comme tout au plus une faute d'orthographe d'usage. L'inventaire de ces erreurs permettra de réévaluer cette sous-catégorie.

\section{ERREUR}

1 - Poliement

2- Joliement

3- Quelques fois

4- Quoi que

5- Asser

6- Toujour

7 - D'avantage

8- Tous généreux

9 - Males vues

10 - Ils sont biens gentils

11 - J'ai peut d'argent

12 - I faut peut être y penser

13 - Le pays ou je suis né

14- Ces gens sont souvents
FORME CORRECTE

Poliment

Joliment

Quelquefois

Quoique

Assez

Toujours

Davantage

Tout généreux

Mal vues

Ils sont bien gentils

J'ai peu d'argent

Il faut peut-être y penser

Le pays où je suis né

Ces gens sont souvent

NOTE: La maîtrise orthographique du mot invariable qu'est l'adverbe repose sur l'identification de la nature adverbiale du mot. Certaines fautes ne peuvent être que des erreurs d'orthographe d'usage, mais en relevant toutes les erreurs d'adverbes pour le besoin du diagnostic, il sera possible de déterminer les orthographes particulières des adverbes. 


\section{ERREUR TYPE:}

$$
\underline{\mathrm{A}}-\grave{\mathrm{A}}
$$

Cette sous-catégorie comprend surtout des fautes relatives à une confusion du verbe avoir (a) et de la préposition (à).

\section{ERREUR}

1 - À quelle heure

2- Quand à lui

3- Parmis la troupe

4- Entres eux

5 - D'en la chambre

6- S'en ce pouvoir

7 - Je suis prêt du but

$8-$

9

\section{FORME CORRECTE}

À quelle heure

Quant à lui

Parmi la troupe

Entre eux

Dans la chambre

Sans ce pouvoir

Je suis près du but 


\section{CONJONCTION DE SUBORDINATION}

\section{ERREUR TYPE:}

Quelle-Qu'elle

Les fautes de cette sous-catégorie sont dues à une méconnaissance de la forme en regard du contexte de son emploi.

\section{ERREUR}

1 - Quelle veut venir

2- S'y tu veux

3- Parceque

4- Quoi que

5- Etant donner

6- Qu'en

$7-$

$8-$

9

$10-$
FORME CORRECTE

Qu'elle veut venir

Si tu veux

Parce que

Quoique

Etant donné

Quand 


\section{CONJONCTION DE COORDINATION}

\section{ERREUR TYPE:}

$$
\underline{\mathrm{Ou}}-\mathrm{Ou}
$$

La faute origine, la plupart du temps, d'une confusion du pronom, de l'adverbe ou de la conjonction de subordination, de forme «où», et de la conjonction de subordination, de forme «ou».

\begin{tabular}{|cll|}
\hline & ERREUR & FORME CORRECTE \\
$1-$ & Je pars si non... & Je pars sinon... \\
$2-$ & C'est à dire & C'est-à-dire \\
$3-$ & Dailleurs & D'ailleurs \\
$4-$ & Les auteurs suivants, soient & Les auteurs suivants, soit \\
$5-$ & Voir même & \\
$6-$ & & Voire même \\
$7-$ & & \\
$8-$ & & \\
$9-$ &
\end{tabular}

1 Ce cas en sera aussi un d'orthographe d'usage (Signes auxiliaires); la décision appartiendra au maître et ce, dépendamment de ce qu'il veut faire acquérir.

2 Contrairement à ce qu'en disent les grammaires, ce mot est le plus souvent employé comme une conjonction de coordination. 


\section{INTERJECTION}

\section{ERREUR TYPE:}

$$
\text { Eu! - Euh! }
$$

Pour cette sous-catégorie, le scripteur ne connaît pas les variations touchant l'écriture de l'interjection. Les cas présentés peuvent être vus comme des cas d'orthographe d'usage, toutefois, les interjections présentent des caractéristiques orthographiques spécifiques, et entre autres celle de l'invariabilité.

\section{ERREUR}

$1-\mathrm{Ha} !$

2- He!

3- Chutte!

4- ciel!

5- Bompté divine!

6- Toudoux!

7- bravo!

8 - He bien!

9- Gard!

10- Suffi!

11- Wais!

12- Ouffe!

13- Krak!

14- A Dieu!

15- Zutte!
FORME CORRECTE

Ah!

Eh!

Chut!

Ciel!

Bonté divine!

Tout doux!

Bravo!

Eh bien!

Gare!

Suffit!

Ouais!

Ouf!

Crac!

Adieu!

Zut! 
Cette dernière sous-catégorie Inclassable sera utile afin de regrouper les erreurs inclassables n'appartenant pas aux sous-catégories précédentes comprises à l'intérieur de la catégorie Morphologie. 


\section{ACCORD}

La catégorie Accord regroupe les fautes qui résultent du choix d'une forme ne marquant pas adéquatement la correspondance entre deux mots dont l'un est subordonné à l'autre.

Cette troisième catégorie comprend sept sous-catégories. De plus, la sous-catégorie Verbe est divisée en huit sous-catégories.

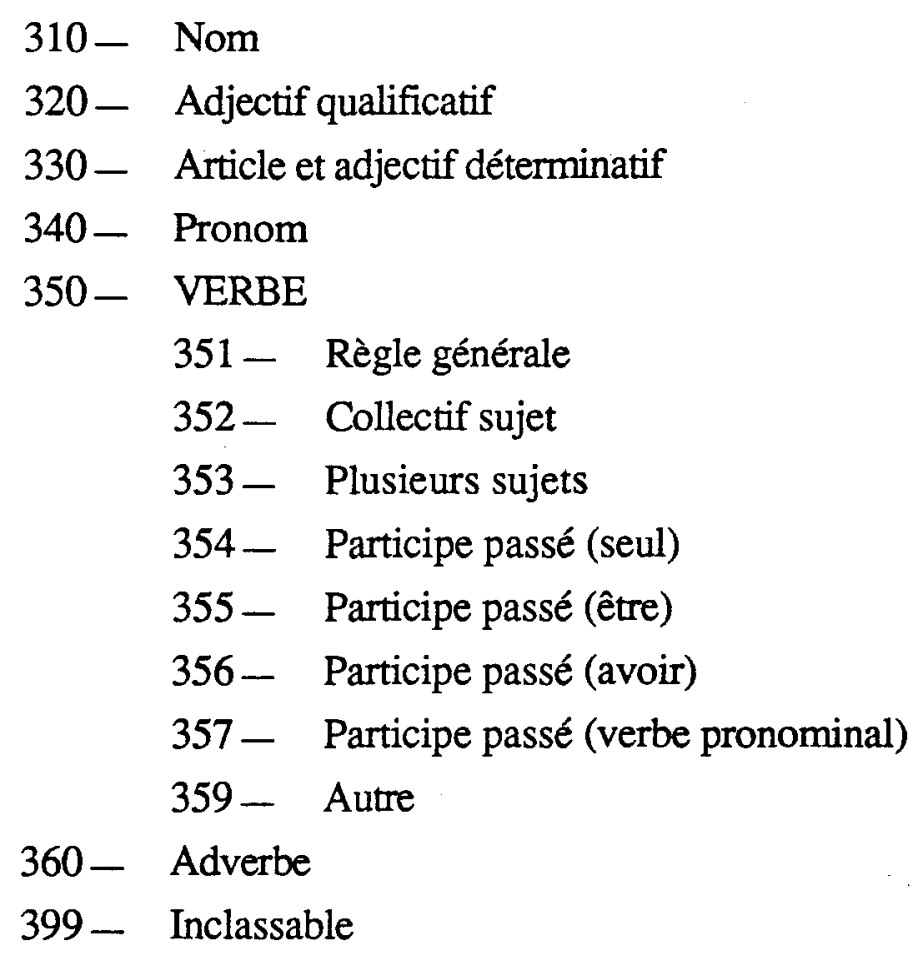




\section{ERREUR TYPE:}

Six million_ - Six millions

Cette sous-catégorie englobe des accords absents ou fautifs du nom commun ou du nom propre. Un rappel de la règle d'accord des noms communs s'impose: «Les noms communs s'accordent en nombre selon le sens de la phrase (Roy, 1995)».

\section{ERREUR}

1 - Des plan divers

2- Ils ont peines à se souvenir

3- Les samedi étoilés

4- Beaucoup de personne

5- Ces trois Isabelles

6- D'autre tension

7 - Un panier de pomme

8- Des fruits de qualités

9- Des tartes aux citrons

10 - Des pommes purées

\section{FORME CORRECTE}

Des plans divers

Ils ont peine à se souvenir

Les samedis étoilés

Beaucoup de personnes

Ces trois Isabelle

D'autres tensions

Un panier de pommes

Des fruits de qualité

Des tartes au citron

Des pommes purée 


\section{ADJECTIF QUALIFICATIF}

\section{ERREUR TYPE:}

De grand enfant_ - De grands enfants

Cette sous-catégorie englobe les fautes réalisées dans l'accord des adjectifs qualificatifs. Ici la règle des adjectifs est la suivante: «Les adjectifs qualificatifs s'accordent en genre et en nombre avec le(s) nom(s) au(x)quel(s) ils se rapportent.»

\section{ERREUR}

1 - Âme léger 1

2 - Cher belle-soeur

3 - Des commentateurs sportif

4 - Feuille bleu

5 - Vie personnel

6 - Promesse électoral

7 - Son origine réel

8 - De façon général

9 - Les points particulier

10 - Une personne instruit
FORME CORRECTE

Âme légère

Chère belle-soeur

Des commentateurs sportifs

Feuille bleue

Vie personnelle

Promesse électorale

Son origine réelle

De façon générale

Les points particuliers

Une personne instruite

1 Ce sera au maître de juger si ce type d'erreur en est un de morphologie du nom (210) ou bien d'accord de l'adjectif (320). 


\section{ARTICLE ET ADJECTIF DÉTERMINATIF}

\section{ERREUR TYPE:}

Tous - Tout

Cette sous-catégorie englobe les déterminants mal choisis ou mal accordés. Les déterminants s'accordent aussi en genre et en nombre avec le(s) nom(s) au(x)quel(s) ils se rapportent.

\section{ERREUR}

1 - Tel mère, tel fille

2 - Quelque personnes

3 - Tout ceux qui la précèdent

4 - Les fondements même

5 - D'autres part

6- Leur valeurs

7 - Quel surprise!

8 - Les raisons auquelle

$9-$

$10-$

\section{FÓRME CORRECTE}

Telle mère, telle fille

Quelques personnes

Tous ceux qui la précèdent

Les fondements mêmes

D'autre part

Leurs valeurs

Quelle surprise!

Les raisons auxquelles 


\section{PRONOM}

\section{ERREUR TYPE:}

Il en meurent - Ils en meurent

Dans cette sous-catégorie, les erreurs sont surtout celles d'un mauvais accord des pronoms personnels, possessifs, démonstratifs et indéfinis.

\section{ERREUR}

1 - Nous-même

2 - Il en veulent

3 - Leurs solitude

4 - Entre autre

5 - Les une des autres

6 - La pierre sur lequel

7 - Chez eux

8 - Quel mouvements

$9-$

$10-$

\section{FORME CORRECTE}

Nous-mêmes

Ils en veulent

Leur solitude

Entre autres

Les unes des autres

La pierre sur laquelle

Chez elles

Quels mouvements 


\section{0 351}

\section{VERBE RÈGLE GÉNÉRALE}

\section{ERREUR TYPE:}

$\underline{\text { Soit }}$ - Soient

La sous-catégorie Règle générale du verbe englobe les formes simples d'accord des verbes. Les fautes contenues dans cette sous-catégorie originent d'un verbe ou mal accordé ou non accordé. Bref, les fautes de cette sous-catégorie sont souvent un accord fautif en personne.

\section{ERREUR}

1 - Les noms représente

2 - Le jeune retrouvent sa place

3 - Dans la tension 1, se trouve les adjectifs $\mathbf{1}$

4 - La place qu'occupe ces mots 1

5 - Les paroles du jeune enfant exprime $^{2}$

6 - La deuxième catégorie des noms sont ${ }^{2}$

7 - On retrouvent ${ }^{3}$

8 - La pensée nous aident ${ }^{2}$

\section{FORME CORRECTE}

Les noms représentent

Le jeune retrouve sa place

Dans la tension 1 , se trouvent les adjectifs

La place qu'occupent ces mots

Les paroles du jeune enfant expriment

La deuxième catégorie des

noms est

On retrouve

La pensée nous aide

$10-$

1 Cet accord montre que le scripteur a inversé le sujet et le complément.

2 Il est net de voir le rôle joué, ici, par les mots «écrans».

3 Cet accord fait référence à des cas de pronoms à pluralité inteme. 


\section{COLLECTIF SUJET}

\section{ERREUR TYPE:}

Une foule de gens dira - Une foule de gens diront

L'accord du collectif sujet relève d'une interprétation fine du sens. Certains emplois limites obligent à accepter l'un ou l'autre accord.

\section{ERREUR}

1 - La plupart des gens a

2- Son peu de qualités l'ont

3- Combien de gens s'imagine

4- Une foule de malades accouraient

5- La plupart est convaincu

6- Plus d'une Mélanie pensèrent

7 - Moins de deux mois est passé

$8-$

$9-$

10

\section{FORME CORRECTE}

La plupart des gens ont

Son peu de qualités l'a

Combien de gens s'imaginent

Une foule de malades accourait

La plupart sont convaincus

Plus d'une Mélanie pensa

Moins de deux mois sont passés 


\section{PLUSIEURS SUJETS}

\section{ERREUR TYPE:}

L'une ou l'autre ont - L'une ou l'autre a

Les types d'erreurs regroupées à l'intérieur de cette sous-catégorie seront souvent ceux aux effets causés, par exemple, par l'utilisation de certains mots particuliers: «ainsi que», «ou», «ni», «l'un et l'autre», etc.

\section{ERREUR}

1 - Ni l'un ni l'autre ne viendront

2- L'un ou l'autre ont

$3-$

$4-$

$5-$

$6-$

$7-$

8

$9-$

$10-$
FORME CORRECTE

Ni l'un ni l'autre ne viendra L'un ou l'autre a 


\section{PARTICIPE PASSÉ (SEUL)}

\section{4}

\section{ERREUR TYPE:}

Mes ouvrages perdu_ - Mes ouvrages perdus

Cette sous-catégorie englobe les erreurs d'accord commises à l'encontre des participes passés employés sans auxiliaire. Il importe de s'assurer qu'en regard du contexte, le mot est bien un participe passé et non un simple adjectif. Toutefois dans les deux cas, la règle sera la même: «Les adjectifs et les participes passés employés sans auxiliaire s'accordent en genre et en nombre avec le(s) nom(s) auxquel(s) ils se rapportent.»

\section{ERREUR}

1 - Les chiens abandonné

2- Des personnes connu du milieu

3- Des meurtres signalé à la police

4- Des animaux maltraité

5- Cette étudiante passionné

6- Une chambre très ordonné

7 - Un coupable interrogé sur place

\section{FORME CORRECTE}

Les chiens abandonnés

Des personnes connues...

Des meurtres signalés à la police

Des animaux maltraités

Cette étudiante passionnée

Une chambre très ordonnée

Un coupable interrogé sur place

$8-$

$9-$

$10-$

NOTE: Dans la très grande majorité des cas, le participe passé peut être employé comme un adjectif ayant souvent un sens beaucoup plus restreint. Toutefois, la présence du complément indiquera la nature, soit de participe passé ou d'adjectif. 


\section{5}

\section{PARTICIPE PASSÉ (ÊTRE)}

\section{ERREUR TYPE:}

Elles sont allé - Elles sont allées

La faute de cette sous-catégorie est commise parce que le scripteur ne connaît pas ou n'a pas su mettre en pratique la règle d'accord des participes passés employés avec l'auxiliaire être: Les participes passés s'accordent en genre et en nombre avec le sujet du verbe.

\section{ERREUR}

1 - Il sera menacés

2 - Les pays sont devenu

3- Les peuples ont été sensibilisé

4- Elle est construit par l'auteur

5- Les filles ont été reconduits

6- Mes amis sont arrivé

7 - Ces étudiantes seront admis

$8-$

9-
FORME CORRECTE

Il sera menacé

Les pays sont devenus

Les peuples ont été sensibilisés

Elle est construite par l'auteur

Les filles ont été reconduites

Mes amis sont arrivés

Ces étudiantes seront admises

$10-$ 


\section{PARTICIPE PASSÉ (AVOIR)}

\section{ERREUR TYPE:}

Les fleurs que j'ai vu﹎-Les fleurs que j'ai vues

Cette sous-catégorie englobe les fautes d'accord commises dans les participes passés employés avec l'auxiliaire avoir. L'accord n'a pas été fait ou a été fait d'une façon fautive.

\section{ERREUR}

1 - Ces filles ont vécues

2- Elle ne m'a pas peiné (fém.)

3 - Les fleurs que j'ai gagné

4- La fillette a eue sa poupée

5 - Le groupe a réussis

6 - La voiture que j'ai vu

$7-$

$8-$

$9-$

$10-$

\section{FORME CORRECTE}

Ces filles ont vécu

Elle ne m'a pas peinée

Les fleurs que j'ai gagnées

La fillette a eu sa poupée

Le groupe a réussi

La voiture que j'ai vue 


\section{ERREUR TYPE:}

Il importe qu'elle se soit aperçu_ - Il importe qu'elle se soit aperçue

«Le participe passé des verbes pronominaux réfléchis ou réciproques s'accorde avec le pronom réfléchi quand celui-ci est l'objet direct (Grevisse, 1980, p.934).»

\begin{tabular}{|lll|}
\hline ERREUR & FORME CORRECTE \\
$1-$ & Ils se sont donné aux adversaires & Ils se sont donnés aux... \\
$2-$ & Ils se sont imposés une règle & Ils se sont imposé une règle \\
$3-$ & Elles se sont demandées & Elles se sont demandé \\
$4-$ & Les chatons se sont lavé & Les chatons se sont lavés \\
$5-$ & Les notes qu'il s'est donné & Les notes qu'il s'est données \\
$6-$ & & \\
$7-$ & & \\
$8-$ & & \\
$9-$ &
\end{tabular}

\section{AUTRE}

Une sous-catégorie Autre est présente pour les erreurs relevées qui ne semblent rejoindre ni l'une ni l'autre des sous-catégories du verbe vues précédemment. De plus, la souscatégorie Autre peut servir au correcteur chez qui un doute a pu s'installer. Ainsi, il sera possible d'éviter un mauvais classement, car il y aura consultation et par après la faute sera transférée dans la sous-catégorie qui convient. 


\section{ADVERBE - TOUT}

\section{ERREUR TYPE:}

Tout prête - Toute prête

Les mots de cette sous-catégorie sont de nature invariable. C'est pourquoi la plupart des erreurs d'accord de ce mot invariable sont à classer comme fautes de morphologie étant donné l'impossibilité d'accord. Sauf pour l'adverbe «tout», dans les cas où la grammaire lui accorde - à tort d'ailleurs - la variabilité.

$$
\begin{array}{cl} 
& \text { ERREUR } \\
1- & \text { Tout prête } \\
2- & \text { Tout confuses } \\
3- & \text { Toute honteuses } \\
4- & \text { Tout raisonnables } \\
5- & \text { Tout femme } \\
6- & \\
7- & \\
8- & \\
9- & \\
10- &
\end{array}
$$

\section{FORME CORRECTE}

Toute prête

Toutes confuses

Toutes honteuses

Toutes raisonnables

Toute femme

NOTE: Les erreurs d'accord des autres mots invariables (interjection, préposition, conjonction de coordination et de subordination) sont à classer comme des fautes de morphologie, étant donné que par définition un mot invariable n'accepte pas la variabilité d'accord. 


\section{9}

\section{INCLASSABLE}

La catégorie 399 Inclassable regroupera les fautes n'ayant pu trouver classification juste aux sous-catégories précédentes et contenues à l'intérieur de la catégorie Accord. 


\title{
STRUCTURE DE LA PHRASE
}

400

Relèvent de cette catégorie toutes les fautes qui touchent à l'organisation de la phrase, exceptions faites des fautes d'accord. Cette catégorie se divise en sept sous-catégories.

\author{
410 - Choix du mode et concordance des temps \\ 420 - Référent \\ 430 - Mot manquant \\ 440 - Mot superflu \\ 450 - Mot(s) mal choisi(s) \\ 460 - Construction fautive \\ 499 - Inclassable
}




\section{0 \\ CHOIX DU MODE ET CONCORDANCE DES TEMPS}

\section{ERREUR TYPE:}

Si j'aurais - Si j'avais

Ces fautes, qui regroupent majoritairement des formes verbales, originent d'un choix fautif du mode ou du temps dans l'élaboration de la phrase.

\section{ERREUR}

1 - Hier, je vais à Montréal.

2 - Demain, j'allais te rencontrer.

3 - Il aime la musique et aimait le sport.

4 - Si j'aurais le temps.

5 - Les problèmes que nous croyons résolus reviennent.

6- Il est possible de croire qu'il faut continuer.

7 - J'aimerais qu'ils peuvent venir.

8 - J'espère que tu puisses te déplacer.

9 - Si complexe est-elle.

\section{FORME CORRECTE}

Hier, je suis allé à Montréal. Demain j'irai te rencontrer. Il aime la musique et aime le sport.

Si j'avais le temps.

Les problèmes que nous. croyions résolus reviennent. Il est possible de croire qu'il faille continuer.

J'aimerais qu'ils puissent venir. J'espère que tu peux te déplacer. Si complexe soit-elle. 


\section{RÉFÉRENT}

\section{ERREUR TYPE:}

Paul a volé Jean; il a porté plainte - Ce dernier a porté plainte

Les fautes de référent sont les fautes occasionnées par un choix fautif de mot eu égard au rapport que le mot entretient avec d'autres mots de la phrase ou d'une phrase précédente. La faute peut également originer du fait que le ou les mots auxquels il est fait référence ne sont pas présents dans le texte. II peut être possible d'apercevoir, par exemple, un pronom qui ne réfère à aucun mot dans la phrase ou le texte. En résumé, la faute de référent tient au fait que le référent est soit fautif, soit absent.

\section{ERREUR}

1 - Pierre a volé Paul; il a porté plainte.

2- La fillette a aperçu une amie. Elle a traversé la rue.

3- Il se livre à l'ivrognerie. Cela le ruinera.

4- Jean a donné à Paul son cadeau.

5- Il a mangé un ananas; il était bon.

6- Le soleil brûlait son visage; ce dernier était trop chaud.

ou

7- Je me rends compte que le temps passe très rapidement. Chaque jours, moi et mon collègue, nous tentons de faire l'entier du travail demandé, mais nous n'avons jamais suffisamment de temps pour le terminer. $\underline{\text { I }}$ (réf. absent) quitte si rapidement les lieux!

8- Sa valeur devient moindre puisque cela (réf. fautif) suit le mouvement.

9- Les lignes du tenseur de la langue sont pleines parce qu'elle (réf. absent) est permanente. 


\section{MOT MANQUANT}

\section{ERREUR TYPE:}

Qu'il ne vienne $(\sqrt{ })-$ Qu'il ne vienne pas

La sous-catégorie Mot manquant reçoit les fautes tenant à l'absence d'un ou de quelques mots indispensables au sens ou à la grammaticalité de la phrase. Quelquefois l'erreur peut être de l'ordre de l'inattention, mais la faute doit quand même être signalée.

\section{ERREUR}

1 - Je suis contre $(\sqrt{ })$ de concilier les études et le sport.

2 - Il peut donc $(\checkmark)$ avoir un retour.

3 - Certains sont moins précis et $(\sqrt{ })$ nécessaires.

4 - Pour aller vers la valeur pleine $(\sqrt{ })$ destination.

5 - Il se veut donc $(\sqrt{ })$ un outil.

6 - De langue et $(\sqrt{ })$ discours.

7 - Soit par expression ou ( $)$ expressivité.

8 - Les noms, les articles et $(\sqrt{ })$ adjectifs.

9 - Elle ne peut $(\sqrt{ })$ avoir froid.

10 - Il n'y $(\sqrt{ })$ que le nom.
FORME CORRECTE

Je suis contre le fait de concilier les études et le sport.

Il peut donc y avoir un retour. Certains sont moins précis et moins nécessaires.

Pour aller vers la valeur pleine de destination.

Il se veut donc être un outil.

De langue et de discours.

Soit par expression ou par expressivité.

Les noms, les articles et les adjectifs.

Elle ne peut pas avoir froid. II n'y a que le nom. 


\section{ERREUR TYPE: \\ C'est pourquoi que - C'est pourquoi}

Dans le cas où un ou plusieurs mots sont inutiles, voire même nuisibles au sens ou à la grammaticalité de la phrase. Ces mots sont classés dans la sous-catégorie Mot superflu.

\begin{tabular}{|c|c|c|}
\hline & ERREUR & FORME CORRECTE \\
\hline $1-$ & $\begin{array}{l}\text { C'est pourquoi qu'un athlète } \\
\text { doit s'entraîner. }\end{array}$ & $\begin{array}{l}\text { C'est pourquoi un athlète } \\
\text { doit s'entraîner. }\end{array}$ \\
\hline $2-$ & $\begin{array}{l}\text { La personne elle doit étudier } \\
\text { pour réussir. }\end{array}$ & $\begin{array}{l}\text { La personne doit étudier pour } \\
\text { réussir. }\end{array}$ \\
\hline $3-$ & Je vais te les dire mes raisons... & Je vais te dire mes raisons... \\
\hline $4-$ & $\begin{array}{l}\text { Tout compte fait la langue } \\
\text { possède vraiment beaucoup } \\
\text { de secrets. }\end{array}$ & $\begin{array}{l}\text { Tout compte fait la langue } \\
\text { possède beaucoup de secrets. }\end{array}$ \\
\hline $5-$ & $\begin{array}{l}\text { On remarque donc ici l'ajout } \\
\text { d'une tension. }\end{array}$ & On remarque donc l'ajout d'une tension. \\
\hline $6-$ & $\begin{array}{l}\text { La différence d'avec la } \\
\text { phrase de base... }\end{array}$ & $\begin{array}{l}\text { La différence avec la phrase } \\
\text { de base... }\end{array}$ \\
\hline $7-$ & $\begin{array}{l}\text { Cependant, il en est à se } \\
\text { demander. }\end{array}$ & Cependant, il est à se demander. \\
\hline $8-$ & $\begin{array}{l}\text { II sera possible de constater et } \\
\text { ainsi que de pouvoir... }\end{array}$ & $\begin{array}{l}\text { Il sera possible de constater et } \\
\text { de pouvoir... }\end{array}$ \\
\hline $9-$ & $\begin{array}{l}\text { Souvent on peut remarquer } \\
\text { comme dans les médias... }\end{array}$ & $\begin{array}{l}\text { Souvent on peut remarquer } \\
\text { dans les médias... }\end{array}$ \\
\hline $10-$ & & \\
\hline
\end{tabular}




\section{$450 \quad$ MOT(S) MAL CHOISI(S)}

\section{ERREUR TYPE:}

Le sujet que je vous entretiens - Le sujet dont je vous entretiens

Cette sous-catégorie permet d'identifier les fautes reliées au problème du choix d'un ou de plusieurs mots qui sont ou, d'une part, inadéquats de par leur fonction ou, d'autre part, inadéquats de par leur signification. Ces erreurs ne doivent toutefois pas être assimilées à des erreurs de lexique (voir catégorie 600, p.67).

\section{ERREUR}

1 - Si nous allons au cinéma et si on va danser.

2 - Le local à Marie.

3 - Elle fait des études.

4 - L'adjectif et le déterminant trouvent son apport...

5 - La complexification de conjonction de coordination.

6 - Par rapport à la langue et le discours.

7 - La généralisation, tant à elle...

8 - Cela permet à l'enfant à s'initier.

9 - Elles sont divisées par trois parties
FORME CORRECTE

Si nous allons au cinéma et si nous allons danser.

Le local de Marie.

Elle étudie.

L'adjectif et le déterminant trouvent leur apport...

La complexification par conjonction de coordination.

Par rapport à la langue et au discours.

La généralisation, quant à elle...

Cela permet à l'enfant de s'initier.

Elles sont divisées en trois parties. 


\title{
CONSTRUCTION FAUTIVE
}

\section{ERREUR TYPE:}

\author{
Tu penses-tu? - Penses-tu?
}

Cette catégorie fait référence aux constructions de phrases incomplètes ou ne correspondant pas aux lois logiques de construction de la phrase et qui, par le fait même, en affectent le sens ou la grammaticalité. À noter que certains emplois peuvent être considérés comme étant des erreurs de niveau de langue (sous-catégorie 650, p.72).

\section{ERREUR}

1 - Quand que j'irai à Toronto.

2 - C'est à cause que ce sujet me plaît.

3 - Tu penses-tu terminer ce travail pour ce soir?

4 - Le tenseur est utilisé aussi pour expliquer la façon dont laquelle le discours s'émet.

5 - A première vue, il est ambigu de croire en l'existence d'un quelconque genre de phrase en voyant une phrase réduite.

6 - Ce court exposé expliquera la nature du tenseur, de quoi il est composé, sa fonction, à quoi il sert ainsi que son utilité l'analyse dans la phrase.

7 - Deuxièmement, la phrase simple étendue. (Phrase incomplète).

8 - D'abord, la phrase simple de base sera tentée d'être expliquée.

\section{FORME CORRECTE}

Lorsque j'irai à Toronto.

C'est parce que ce sujet me plaît.

Penses-tu terminer ce travail pour ce soir? 


\section{9}

\section{INCLASSABLE}

Cette dernière sous-catégorie permet de regrouper toutes les fautes inclassables. Ces fautes n'appartiennent ni à l'une ni à l'autre des sous-catégories de la catégorie Structure de la phrase, tout en semblant relever de la catégorie. 


\section{SIGNES DE PONCTUATION ET CONVENTIONS}

Cette catégorie regroupe toutes les fautes de ponctuation et de conventions relatives aux guillemets, à la majuscule, à la minuscule, au trait d'union et à l'apostrophe d'élision. Il importe toujours de choisir la sous-catégorie en fonction de la bonne réponse et non en fonction de l'erreur commise. Dans l'exemple «Le chat; le chien et le cheval», la faute sera classée à l'intérieur de la sous-catégorie Virgule de reprise (521) et non à l'intérieur de la sous-catégorie Point-virgule (514).

La catégorie Ponctuation et majuscule est subdivisée en sept sous-catégories. À noter que les sous-catégories Point et Virgule se subdivisent à leur tour en six et en cinq autres souscatégories.

$$
\begin{aligned}
510- & \text { POINT } \\
& 511-\text { Point final } \\
& 512-\text { D'interrogation } \\
& 513-\text { D'exclamation } \\
& 514-\text { Point-virgule } \\
& 515-\text { Deux-points } \\
& 519-\text { Point superflu } \\
520- & \text { VIRGULE } \\
& 521-\quad \text { De reprise } \\
& 522-\quad \text { D'explication } \\
& 523-\quad \text { D'inversion } \\
& 524-\quad \text { D'ellipse et de retrait } \\
& 529-\quad \text { Virgule superflue } \\
530- & \text { Guillemets et parenthèses } \\
540- & \text { Majuscule, minuscule et capitale } \\
550- & \text { Trait d'union } \\
560- & \text { Apostrophe d'élision } \\
599- & \text { Inclassable }
\end{aligned}
$$


La sous-catégorie Point regroupe les sous-sous-catégories des différents types d'usage du point, soit le point final, le point d'interrogation, le point d'exclamation, le point-virgule et les deux-points.

\section{1}

\section{POINT FINAL}

\section{ERREUR TYPE}

$$
\text { Etc } \ldots \text { - Etc. }
$$

«Le point indique la fin d'une phrase. Il se place aussi après tout mot écrit en abrégé (Grevisse, 1969, p.271).» Lorsqu'une phrase est trop longue, il sera considéré qu'il y a erreur où il est indiqué de la séparer. (Dans ce cas, il y aura aussi une faute à classer dans la sous-catégorie 540: Majuscule.) De plus, si le point est absent ou apparaît erronément, cette faute sera marquée. Lorsqu'un mot est écrit en abrégé, un point doit, le plus souvent, apparaître.

\section{ERREUR}

$1-\mathrm{pl}$

2 - adj

$3-\mathrm{cm}$.

4 - etc...

5 - Or, une tension ne peut contenir qu'un seul sujet,

6 - Cette loi est logique!

7 - Il importe de se demander ce qu'est le système?

\section{FORME CORRECTE}

pl.

adj.

$\mathrm{cm}$

etc.

Or, une tension ne peut contenir qu'un seul sujet.

Cette loi est logique.

Il importe de se demander ce qu'est le système.

$8-$

$9-$ 


\section{POINT D'INTERROGATION}

\section{CAS TYPE:}

Veux-tu venir avec moi ce soir?

NOTE: Les exemples, de cette sous-catégorie, relèvent des emplois corrects.

«Le point d'interrogation s'emploie après toute phrase exprimant une interrogation directe (Grevisse, 1969, p.271).»

Lorsque le scripteur a omis d'inscrire cette ponctuation à sa phrase interrogative, c'est à cette sous-catégorie que la faute doit être classée. Toutefois, s'il a introduit un point d'interrogation inutilement, nécessairement un point (ou autre ponctuation) doit le remplacer; la faute sera alors classée à la sous-catégorie Point final (510).

\section{EXEMPLE}

1 - Où est Susie?

2 - Que se passe-t-il?

3 - Quand viendras-tu?

4- Quels sont les activités de ce cours?

$5-$ 


\section{POINT D'EXCLAMATION}

\section{CAS TYPE:}

Quelle histoire!

NOTE: Les exemples, de cette sous-catégorie, relèvent des emplois corrects.

Le point d'exclamation s'inscrit après une exclamation. Les règles de correction sont les mêmes que pour le point d'interrogation.

$$
\begin{array}{ll} 
& \text { EXEMPLE } \\
1- & \text { Hélas! } \\
2- & \text { Je suis tellement heureuse! } \\
3- & \text { Venez les enfants! } \\
4-\quad \text { Bravo! } \\
5-\quad \text { J'ai réussi mon examen! }
\end{array}
$$




\section{POINT-VIRGULE}

\section{CAS TYPE:}

Il prend un verre, une bouteille; il s'assied.

NOTE: Les exemples, de cette sous-catégorie, relèvent des emplois corrects.

«Le point-virgule marque une pause de moyenne durée. Il s'emploie pour séparer, dans une phrase, les parties dont une au moins est déjà subdivisée par la virgule, ou encore pour séparer des propositions de même nature qui ont une certaine étendue (Grevisse, 1969, p.273).»

A - «On utilise le point-virgule pour séparer des propositions de même nature (Brousseau et al., 1991, p.125).»

Ex: Manon est l'amie de Nicole; Nicole est la cousine de Pierre.

B - «On utilise le point-virgule pour séparer, dans une même phrase, des propositions dont l'une au moins comprend déjà une ou des virgules (Brousseau et al., 1991, p.125).»

Ex: Vers huit heures, il est parti; les autres sont restés. 
C - «On utilise le point-virgule pour séparer des propositions exprimant l'opposition, le contraste ou la comparaison (Brousseau et al., 1991, p.125).»

Ex: La rue Racine est une rue ordinairement très achalandée; pourtant on n'y trouve personne après $18 \mathrm{~h}$.

D - «On utilise le point-virgule pour séparer les éléments d'une énumération (Brousseau et al., 1991, p.125).» Note: L'emploi usuel est d'utiliser ici des virgules.

Ex: Pour obtenir votre passeport, il vous faudra: deux photos; un certificat de naissance; une attestation de citoyenneté; une signature. 


\section{DEUX-POINTS}

CAS TYPE:

Il m'a dit: «Je ne veux pas te voir.»

NOTE: Les exemples, de cette sous-catégorie, relèvent des emplois corrects.

A - «On utilise les deux-points pour marquer un rapport logique entre deux parties d'un énoncé (faire suivre d'une minuscule) (Brousseau et al., 1991, p.126).»

Ex: Paul ne pourra pas venir: il habite trop loin.

B - «On utilise les deux-points pour annoncer une énumération (faire suivre d'une minuscule) (Brousseau et al., 1991, p. 126).»

Ex: Je retrouve tous mes amis à cette fête: Paul, Marie, Claude et Lise.

$\mathrm{C}$ - «Ils sont aussi utilisés pour introduire une citation ou un discours direct (faire suivre de guillemets, mais la citation commence par une majuscule), (Brousseau et al., 1991, p.126).»

Ex: Je lui ai dit: «Comment vas-tu?» Il m'a répondu: «Je vais bien.» 
D - «On n'emploie qu'une seule fois les deux-points dans la même phrase, sauf quand on rapporte une citation qui comprend déjà un deux-points (Brousseau et al., 1991, p.126).»

Ex: Georges répondit: «Je ne viendrai pas: je suis pris ce jour-là.»

Une sous-catégorie Point superflu a été ajoutée pour le ou les points superflus. 


\section{VIRGULE}

La virgule sert à marquer une petite pause à la phrase. Évidemment, plusieurs règles s'imposent et se doivent d'être respectées dans l'écriture. Pour ce faire, il est essentiel de distinguer les différentes sortes de virgules et c'est pourquoi cette souscatégorie contient cinq autres sous-catégories.

$$
\begin{array}{ll}
521- & \text { De reprise } \\
522- & \text { D'explication } \\
523- & \text { D'inversion } \\
524- & \text { D'ellipse et de retrait } \\
529- & \text { Virgule superflue }
\end{array}
$$

NOTE: Tous les exemples, relatifs aux emplois des virgules, relèvent aussi des emplois corrects. 


\section{VIRGULE DE REPRISE}

\section{CAS TYPE:}

Jean, Joseph et Marie.

La virgule de reprise s'emploie généralement pour marquer les reprises d'éléments semblables. Ces éléments peuvent être des noms, des verbes, des adjectifs, des sujets, des propositions, etc.

A - Cette virgule est utilisée pour séparer les mots ou groupes de mots dont la reprise n'est pas portée par une conjonction de coordination.

1 - Le français, les mathématiques, la géographie et l'histoire sont les matières que je préfère.

2 - J'ai visité les palais, les boutiques, les églises, etc.

B - Elle est utilisée pour séparer plusieurs propositions coordonnées de même nature.

1 - Je travaille trop, je n'ai pas le temps pour mes loisirs.

2 - Plus on est nombreux, plus on s'amuse. 


\section{VIRGULE D'EXPLICATION}

\section{CAS TYPE:}

Paris, capitale de la France, est une ville très charmante.

La virgule d'explication est généralement employée pour détacher tout élément ayant une valeur explicative.

A - Cette virgule est utilisée pour séparer tout élément ayant une valeur explicative, ce qui est fait le plus souvent à l'aide de deux virgules.

1 - A treize ans, bientôt quatorze, elle paraissait en avoir dix-huit.

2 - Annie, la cousine, était venue rendre visite à Lisa pour son anniversaire.

B - Elle est utilisée pour détacher une proposition explicative.

1 - Bernard, qui attendait son ami, fut surpris par la tempête.

2 - Ma grand-mère, issue d'une famille nombreuse et pauvre, n'a pu faire de longues études.

C - Elle est utilisée pour marquer la valeur explicative des propositions introduites par une conjonction de coordination autre que «et», «ou», «ni».

1 - Je ne peux oser faire ce geste, car les conséquences pourraient en être graves.

2 - Il y a une raison, mais je ne peux la trouver. 


\section{VIRGULE D'INVERSION}

\section{CAS TYPE:}

Ce soir, il regarde la lune.

La virgule d'inversion est généralement utilisée pour marquer l'inversion de structure (complément circonstanciel, proposition circonstancielle, etc.) dans une phrase.

A - Cette virgule est utilisée pour signaler l'inversion affectant un complément circonstanciel placé en tête de phrase.

1 - Après le dîner, les jeunes étaient allés danser.

2 - Après le mariage, nous vous convions à une réception.

B - Elle est utilisée pour signaler l'inversion affectant une proposition circonstancielle placée en tête de phrase.

1 - Avant qu'il n'arrive, je pleurais.

2 - À chaque explosion, je frissonnais sur ma chaise. 


\author{
CAS TYPE: \\ En effet, je désire occuper ce poste. \\ Trois minutes, monsieur!
}

La virgule d'ellipse et de retrait est utilisée pour placer certains mots ou groupes de mots en retrait. Elle sert à isoler un ou des mots mis en apostrophe ou à marquer l'élision d'un mot.

A - La virgule est utilisée pour isoler les mots mis en apostrophe.

1 - Chien, rentre dans ta niche!

2 - Grammaire, tu nous étourdis.

B - Elle est utilisée pour séparer la proposition incise.

1 - Il me plaît, voyez-vous, d'assister à ce spectacle.

2 - Un instant, dit-il, je suis occupé.

C - Elle est utilisée pour marquer l'ellipse d'un verbe ou d'autres mots.

1 - Le vent était doux et le soleil, brûlant.

2 - Paul s'amuse au parc et Martha, à la piscine. 


\section{9}

\section{VIRGULE SUPERFLUE}

Une sous-catégorie Virgule superflue a été prévue pour la ou les virgules superflues ne correspondant ni à 521, ni à 522, ni à 523, ni à 524 . 


\section{GUILLEMETS}

\section{CAS TYPE:}

Il m'a dit: «Je dois étudier mon examen.»

NOTE: Les exemples, de cette sous-catégorie, relèvent des emplois corrects.

«Les guillemets s'emploient pour encadrer une citation ou un discours direct (Grevisse, 1969, p.274).»

Les auteurs du livre Le français pour l'essentiel (1991) indiquent qu'il y a trois façons de rapporter les paroles d'une personne, dont deux utilisent les guillemets.

A- «Dans le style direct, on reproduit une citation relativement courte en la faisant précéder et suivre des guillemets ouvrants et fermants, et en recourant à une proposition incise qu'on place avant (avec les deux-points) ou après la citation, ou encore au milieu de celle-ci (Brousseau et al., 1991, p.169).»

Ex: - Elle m'a dit: «Je ne peux pas te le dire.»

- «Je ne peux pas te le dire», m'a-t-elle dit.

- «Je ne peux pas, m'a-t-elle dit, te le dire.»

B- «Dans le style indirect, on peut fondre une citation à l'intérieur d'une phrase en recourant aux guillemets d'usage et sans utiliser de ponctuation devant les guillemets ouvrants (Brousseau et al., 1991, p.169).»

Ex: - Il dit qu' «il est un expert en ski» et que «ce sport lui procure beaucoup de plaisir». 


\section{MAJUSCULE, MINUSCULE ET CAPITALE}

\section{CAS TYPE:}

La Ville de Québec.

NOTE: Les exemples, de cette sous-catégorie, relèvent des emplois corrects.

«La majuscule remplit deux fonctions principales. Dans la phrase, elle met en valeur certains changements d'ordre matériel ou intellectuel; dans les noms propres, elle souligne le caractère unique, singulier ou supérieur de ces noms (Brousseau et al., 1991, p.51).»

A - Une majuscule s'emploie au début d'une phrase.

B - Une majuscule est utilisée après un point pour introduire une autre phrase.

C - Une majuscule s'emploie après les points d'interrogation [?], d'exclamation [!] et de suspension [...], si ces points terminent une phrase.

D - Une majuscule est présente après les deux-points [:] s'ils sont suivis d'une phrase complète et d'un énoncé complet cité avec ou sans guillemets.

Ex: Je lui ai demandé: «Avez-vous eu du plaisir?»

E - Une majuscule est utilisée pour les noms de personnes et d'animaux (prénoms, noms de famille, surnoms, diminutifs).

Ex: Paul Leblanc, le chien Brutus, Ti-Coq. 
F - Les noms de peuples, de races, de groupes ethniques, d'habitants d'une région ou d'une ville commencent par une majuscule.

Ex: Les Canadiens français (nom + adj.).

Ce sont des Québécois. Mais «le peuple québécois (adj.)».

Il n'y a pas que des Noirs en Afrique.

$\mathrm{G}$ - Une majuscule s'emploie pour désigner des termes spécifiques (termes désignant le lieu lui-même).

Ex: La ville de Montréal. La région du Saguenay-Lac-St-Jean.

Rivière-des-Prairies (municipalité), mais la rivière des Prairies (rivière).

$\mathrm{H}$ - Les mots «ville», «cité» et «municipalité» prennent une majuscule quand ils désignent un corps administratif ou un service public.

Ex: La Ville de Québec (corps administratif) a haussé les taxes.

La ville de Québec est au bord du St-Laurent.

Il existe beaucoup d'autres cas et de variables pour lesquels il y a obligation d'utiliser une majuscule. Comme la liste en serait trop longue, seuls les cas les plus fréquents ont été présentés ici. La consultation d'une grammaire sera utile pour des cas, tels que les points cardinaux, les noms de jours et de mois, les noms désignant une période historique, le mot «état», le mot «église», les organismes gouvernementaux, les mots «Ministre» et «ministère», les noms de partis politiques, les noms de membres des partis, le premier mot d'un titre, les noms de logiciels, le nom d'une entité administrative, les mots «collège» et «université», etc. 


\section{TRAIT D'UNION}

\section{ERREUR TYPE:}

\section{Sommes nous - Sommes-nous}

La plupart de ces fautes apparaissent dans la construction d'une phrase et dans l'inversion d'un verbe. Il importe de ne pas confondre cette sous-catégorie avec celle des Signes auxiliaires (sous-catégorie 120).

\section{ERREUR}

1 - Êtes vous des étudiants?

2 - Peut être que je viendrai.

3 - La leçon peut-être intéressante.

4 - Cette chose là!

5 - Que remarque t'il?

6 - Celle ci

7 - Vu ci haut

8 - Donne m'en un peu.

$9-$

$10-$

11

12

$13-$

14

\section{FORME CORRECTE}

Êtes-vous des étudiants?

Peut-être que je viendrai.

La leçon peut être intéressante.

Cette chose-là!

Que remarque-t-il?

Celle-ci

Vu ci-haut

Donne-m'en un peu. 


\section{APOSTROPHE D'ÉLISION}

\section{ERREUR: TYPE:}

Si il vient - S'il vient

L'apostrophe s'utilise pour marquer l'élision de certaines voyelles.

\section{ERREUR}

1 - La lecture de d'autres recherches.

2 - Je me demande si il viendra.

3 - De une amitié sincère.

4 - Lorsque l'on cherche.

5 - Puisque elle viendra.

6 - De un combat.

$7-$

$8-$

$9-$

$10-$

11

$12-$

$13-$

\section{FORME CORRECTE}

La lecture d'autres recherches.

Je me demande s'il viendra.

D'une amitié sincère.

Lorsqu'on cherche.

Puisqu'elle viendra.

D'un combat. 
Une sous-catégorie Inclassable a été prévue pour les cas particuliers occasionnant un questionnement ou une incertitude. Si une faute ne correspond à aucune des sous-catégories de la catégorie Ponctuation et conventions, elle sera déterminée inclassable. De plus, si le correcteur hésite à placer la faute dans l'une ou l'autre des sous-catégories; il vaudra mieux l'enregistrer à la sous-catégorie 599 et une consultation pourra alors se faire avec les autres correcteurs pour classer, si possible, plus adéquatement la faute. 


\section{LEXIQUE}

Cette sixième catégorie relève toutes les fautes qui portent sur le choix lexical des mots. Elle regroupe six sous-catégories.

610 - Impropriété

620 - Accord conceptuel

630 - Anglicisme

640 - Barbarisme, pléonasme, tautologie

650 - Niveau de langue

699 - Inclassable 


\section{ERREUR TYPE:}

Le lac est creux — Le lac est profond.

Cette sous-catégorie regroupe les erreurs relatives aux emplois impropres d'un mot ou d'un groupe de mots ne traduisant pas de façon satisfaisante le sens recherché ou attendu.

\begin{tabular}{|lll|}
\hline & ERREUR & FORME CORRECTE \\
$1-$ & Le schéma démontre ... & Le schéma montre ... \\
$2-$ & Je suis venu tôt ... & Je suis arrivé tôt ... \\
$3-$ & Mon côté se situe ... & Mon point de vue se situe ... \\
$4-$ & Au travers des étudiants ... & Parmi les étudiants ... \\
$5-$ & Il l'a joint ... & Il l'a rejoint ... \\
$6-$ & & \\
$7-$ & & \\
$8-$ & & \\
$9-$ & & \\
$10-$ & & \\
\end{tabular}




\section{ACCORD CONCEPTUEL}

\section{ERREUR TYPE:}

Demander une question - Poser une question

Cette sous-catégorie comprend les fautes dont un mot ne convient pas avec les autres mots utilisés dans la phrase.

ERREUR

1 - Les propos développés.

2 - Le texte dit.

3 - Des constructions de phrases à reformuler.

4 - Les observations qui peuvent être remarquées.

5 - Je fais confiance en mon but.

6 - La synchronie parle de l'évolution.

$7-$

$8-$

$9-$

$10-$

$11-$

$12-$

$13-$

$14-$

FORME CORRECTE 


\section{ANGLICISME}

\section{ERREUR TYPE:}

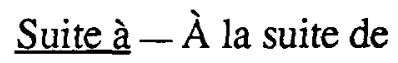

Cette sous-catégorie englobe ou les termes, ou les expressions, ou les mots empruntés à la langue anglaise.

\section{ERREUR}

1 - C'est l'fun

2- Jusqu'à date

3- Gang

4 - Prendre contact

5 - Faire sens

6 - O.K.

7 - Suite à

8 - Connaître (savoir)

9 - Faire sens

10 - Je cherche pour toi

11 - En fait
FORME CORRECTE

C'est plaisant

Jusqu'ici, jusqu'à maintenant

Bande, groupe

Rejoindre

Avoir du sens

D'accord

À la suite de

Savoir

Avoir du sens

Je te cherche

De fait 


\section{BARBARISME, PLÉONASME ET TAUTOLOGIE}

640

ERREUR TYPE:

Séductible - Enjôleur

Cette sous-catégorie regroupe les erreurs relatives à l'utilisation d'une forme inexistante ou non acceptée.

\section{ERREUR}

1 - Dégacer

2 - Motivationnel

3 - Inormal

4 - Boucanne

5 - Tabagiste

6 - Séductible

7 - Pogner quelque chose

8 - Se virer de bord

9 - Se tasser

$10-$ 


\section{ERREUR TYPE:}

«Coudon»

Cette sous-catégorie fait référence aux termes utilisés en langue orale qui ne peuvent être acceptés en langue écrite. Le message écrit doit respecter certaines lois. De plus, les phrases ainsi que les termes doivent correspondre au discours écrit. Les termes du niveau familier ou populaire sont aussi à classer à l'intérieur de cette sous-catégorie.

Constructions de phrases prenant leur source à l'oral:

Termes à éviter:
- Le français, moi, je l'écris mal.

- Les profs, eux, ils croient qu'ils ont toujours raison.

- Du temps, voyons, est-ce que j'en ai moi, pour penser à cette affaire-là.

- J'ai été à Dolbeau.

$$
\begin{array}{ll}
\text { - Là, là. } & \text { - Ça } \\
\text { - C'est cool! } & \text { - Oups! } \\
\text { - Eh bien! } & \text { - Hélas! } \\
\text { - C'est l'bout! } & \text { - Super! }
\end{array}
$$

Si le correcteur juge que le choix du ou des mots ne convient pas, mais ne correspond à aucune des sous-catégories vues précédemment, l'erreur sera inscrite à la sous-catégorie Inclassable. 


\section{STYLISTIQUE}

\section{0}

Robert (1967) définit la stylistique comme étant «une connaissance pratique des particu-

larités de style propres à une langue». À l'intérieur de cette sous-catégorie, on relèvera ou des expressions, ou des emplois, ou des mots présentant quelque faiblesse de style. Cette catégorie se subdivise en trois sous-catégories.

710 - Précision de la langue

720 - Concision de la langue

730 - Harmonie de la langue 


\title{
710 PRÉCISION DE LA LANGUE
}

\author{
ERREUR TYPE: \\ Il $\underline{a}$ un poste - Il occupe un poste
}

La sous-catégorie Précision de la langue veut informer le scripteur si sa langue est claire, si elle porte bien le propos, si les mots sont bien choisis et si les termes sont précis. Ces fautes font souvent référence à des phrases ou à des mots imprécis, incompréhensibles, drôlement présentés, embrouillés, etc.

\section{ERREUR}

1 - J'ai eu un prix.

2 - Ma mère est la chose que...

3 - Les opinions sont les mêmes.

4 - Il a un poste.

5 - Le sujet est avant le verbe

$6-$

$7-$

$8-$

9

$10-$

\section{FORME CORRECTE}

J'ai remporté un prix.

Ma mère est la personne que...

Les opinions concordent.

Il occupe un poste.

Le sujet précède le verbe 


\section{CONCISION DE LA LANGUE}

\section{ERREUR TYPE:}

Une très infime partie - Une infime partie

Cette sous-catégorie touche la netteté de la langue. Généralement, les fautes seront de l'ordre d'une langue compliquée, manquant de concision, de simplicité ou présentant une redondance.

\section{ERREUR}

1 - Au grand maximum.

2 - Une très infime partie.

3 - Une hémorragie de sang.

4- Monter en haut.

5- Au jour d'aujourd'hui.

6- Prévoir à l'avance.

7

$8-$

9

10

$11-$

$12-$

$13-$

$14-$

\section{FORME CORRECTE}

Au maximum.

Une infime partie.

Une hémorragie.

Monter.

Aujourd'hui.

Prévoir. 


\section{HARMONIE DE LA LANGUE}

\section{ERREUR TYPE:}

«Il préfère faire ses affaires seul.»

Cette dernière sous-catégorie, relative au style, indique si la langue est belle, coulante, agréable, originale; bref, si elle est harmonieuse. Le correcteur précisera à cet endroit si la langue est lourde, gênante ou ennuyante. Aussi, si la langue est cacophonique ou cacographique. L'harmonie de la langue tente de relever le style bizarre ou l'écriture maladroite, ainsi que certaines erreurs de disposition et d'ordre des mots.

\section{ERREUR}

1 - Il préfère faire ses affaires seul.

2 - En en entendant parler.

3 - À l'exception de la dernière, l'Académie accepta toutes les acceptations nouvelles du terme.

4- J'ouvris la fenêtre de ma chambre sur la nuit.

$5-$

$6-$

$7-$

$8-$

$9-$

$10-$ 


\section{QUALITÉ TEXTUELLE}

\section{0}

Cette catégorie réfêre aux règles d'écriture et d'organisation d'un texte. Elle présente trois sous-catégories, lesquelles, à leur tour, présentent plusieurs sous-sous-catégories.

810- RÈGLES D'ORGANISATION

811- Introduction au thème

812- Introduction du thème

813- Présentation du plan

814- Paragraphe

815- Développement

816- Conclusion

820- RÈGLES DE SOUTIEN

821- Transition

822- Citation

823- Schéma, figure, tableau

824- Titre et sous-titre

825- Référence

830- RÈGLES DU GENRE 


\section{RÈGLES D'ORGANISATION}

Cette sous-catégorie réfêre aux règles d'organisation du texte, c'est-à-dire à ses grandes parties, dont l'introduction, les paragraphes, le développement et la conclusion. Les fautes seront relevées en regard des lois qui régissent les différentes parties de la structure d'un texte.

\section{INTRODUCTION AU THÈME}

Cette partie du texte consiste à amener le sujet à l'aide d'une idée générale en rapport étroit avec le sujet. Le correcteur devra vérifier si le sujet ou le thème est adéquatement amené.

\section{2}

\section{INTRODUCTION DU THÈME}

Cette sous-catégorie réfère au passage servant à poser le sujet; à le présenter soit dans sa formulation originale, soit dans ses propres mots. Il s'agira de signaler si le thème est adéquatement présenté. 


\section{PRÉSENTATION DU PLAN}

813

Cette autre sous-catégorie réfère à la division du sujet en introduction, c'est-à-dire à la présentation des grandes parties du développement. De plus, cette sous-catégorie réfère à l'enchaînement des deux ou trois idées principales du plan présentées tout au long du texte. Cette présentation se fait naturellement et avec logique. Si cette partie est présente, il sera aussi essentiel de juger si elle convient.

\section{PARAGRAPHE}

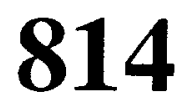

Généralement, il y a une idée principale par paragraphe. Un paragraphe comporte aussi une idée énoncée, illustrée, expliquée et conclue. L'équilibre doit s'appliquer quant à la longueur des paragraphes. De plus, un paragraphe doit habituellement contenir plus d'une phrase.

\section{DÉVELOPPEMENT}

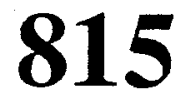

La sous-catégorie du développement représente l'exposé détaillé du sujet. Il faut donc juger si le sujet est bien développé. De plus, il importe que chacune des parties laisse voir un enchaînement logique et que les idées laissent voir une certaine progression. Toutefois, la plupart des erreurs relatives au développement relèveront plutôt de la catégorie 900, Qualité de la pensée. 


\section{6}

\section{CONCLUSION}

Cette partie permet de faire voir l'unité d'ensemble du travail. Elle doit amener une réponse au problème de départ et facultativement ouvrir et élargir le propos. Le correcteur devra se demander si la conclusion permet d'apercevoir l'unité d'ensemble du travail et si elle fait bien une synthèse de ce qui a été traité.

\section{RÈGLES DE SOUTIEN}

Cette sous-catégorie regroupe tous les éléments et les lois d'écriture rattachés au développement d'un texte, tels que les transitions, les citations, les schémas, les figures, les tableaux, les titres et sous-titres et finalement les références.

\section{TRANSITION}

À l'intérieur du développement, les liaisons marquent l'enchaînement logique. Elles assurent la liaison des différentes parties et la bonne articulation du texte et des idées. Si les transitions manquent ou laissent à désirer, il importe de le souligner. 


\section{CITATION}

822

Dans le cas où le scripteur n'aurait pas cité honnêtement ses sources, il faut lui indiquer cette maladresse. Les noms des auteurs doivent être donnés et présentés selon les règles. Il importe d'indiquer chez quel auteur les idées sont puisées.

\section{SCHÉMA, FIGURE, TABLEAU}

823

Cette sous-catégorie réfère aux schémas, figures et tableaux importants à la compréhension d'un texte. Ces additions sont nécessaires pour aider à la compréhension du lecteur. Elles doivent être complètes et exemptes d'erreurs. Elles doivent être bien amenées et bien présentées à l'aide d'une phrase complète. Les schémas doivent être placés à l'intérieur même du texte, puis expliqués.

\section{TITRE ET SOUS-TITRE}

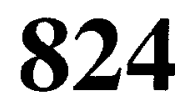

Cette sous-catégorie réfère à la présentation des titres. Le correcteur se demandera si les titres sont clairs et précis et s'ils sont utilisés à bon escient. 
Cette sous-catégorie réfère à la façon de citer les références. Les références doivent être présentes et clairement indiquées.

Comme il existe plusieurs styles d'écriture pouvant être attribués à un texte, les critères de cette catégorie devront changer selon le style employé. Pour une dissertation scientifique, le style objectif sera exigé. Le compte rendu préférera aussi le style objectif; toutefois un style plus personnel pourra se justifier. Pour le style objectif d'écriture, il sera requis:
a) d'être objectif;
b) d'exposer simplement les faits;
c) de n'émettre aucun sentiment;
d) de ne pas utiliser les pronoms «je», «nous», «on», «tu» et
e) d'éviter les questions directes. 


\section{QUALITÉ DE LA PENSÉE}

\section{0}

Cette dernière grande catégorie réfêre aux idées présentées dans un texte. La qualité de la pensée et du propos est le critère fondamental d'évaluation d'un texte. Cette catégorie comporte trois sous-catégories.

910 - Choix et netteté des idées

920 - Logique et enchaînement des idées

930 - Profondeur et originalité 


\section{CHOIX ET NETTETÉ DES IDÉES}

Cette sous-catégorie touche l'orientation du sujet. Le choix des idées doit être net, clair et précis. Lorsque les idées ne sont pas bien choisies, c'est à cette sous-catégorie qu'il importe de placer la faute. Le correcteur devra juger si le texte répond à la question, s'il est exempt d'inexactitudes théoriques, si tous les points à traiter sont couverts et si les exemples sont bien choisis.

\section{0 \\ LOGIQUE ET ENCHAÎNEMENT DES IDÉES}

Cette partie fait référence à l'articulation du texte. Le correcteur s'attardera davantage à la qualité du développement, à l'articulation du raisonnement et à la logique d'ensemble du texte.

\section{0}

\section{PROFONDEUR ET ORIGINALITÉ}

L'originalité d'un texte tient souvent à la maîtrise des idées et du contenu. Le correcteur doit juger si le texte fait la preuve d'une grande compréhension, si le texte comporte des développements originaux et si le texte fait la preuve d'une grande maîtrise du contenu et de la pensée. 


\section{GRILLE DE CORRECTION}

\begin{tabular}{l|l|}
\hline 100 & ORTHOGRAPHE D'USAGE \\
110 & Cas général \\
120 & Signes auxiliaires \\
130 & Géminée \\
199 & Inclassable \\
\hline 200 & MORPHOLOGIE \\
210 & Nom \\
220 & Adjectif qualificatif \\
230 & Article et adj. déterminatif \\
240 & Pronom \\
250 & Verbe \\
260 & Adverbe \\
270 & Préposition \\
280 & Conjonction de subordination \\
290 & Conjonction de coordination \\
295 & Interjection \\
299 & Inclassable \\
\hline
\end{tabular}

\begin{tabular}{|ll|}
\hline 300 & ACCORD \\
310 & Nom \\
320 & Adjectif qualificatif \\
330 & Article et adj. déterminatif \\
340 & Pronom \\
& \\
350 & VERBE: \\
351 & Règle générale \\
352 & Collectif sujet \\
353 & Plusieurs sujets \\
354 & Part. passé (seul) \\
355 & Part. passé (être) \\
356 & Part. passé (avoir) \\
357 & Part. passé (v. pron.) \\
359 & Autre \\
& \\
360 & Adverbe \\
399 & Inclassable \\
\hline
\end{tabular}

\begin{tabular}{|ll}
\hline 400 & STRUCTURE DE LA PHRASE \\
410 & Choix du mode et concordance des temps \\
420 & Référent \\
430 & Mot manquant \\
440 & Mot superflu \\
450 & Mot(s) mal choisi(s) \\
460 & Construction fautive \\
499 & Inclassable \\
\hline
\end{tabular}

\begin{tabular}{|ll|}
\hline 500 & SIGNES DE PONCTUATION ET \\
& CONVENTIONS \\
510 & POINT \\
511 & Point final \\
512 & D'interrogation \\
513 & D'exclamation \\
514 & Point-virgule \\
515 & Deux-points \\
519 & Point superflu \\
& \\
520 & VIRGULE: \\
521 & De reprise \\
522 & D'explication \\
523 & D'inversion \\
524 & D'ellipse et de retrait \\
529 & Virgule superflue \\
& \\
530 & Guillemets et parenthèses \\
540 & Majuscule, minuscule et capitale \\
550 & Trait d'union \\
560 & Apostrophe d'élision \\
599 & Inclassable \\
\hline
\end{tabular}

\begin{tabular}{|ll|}
\hline 600 & LEXIQUE (vocabulaire) \\
610 & Impropriété \\
620 & Accord conceptuel \\
630 & Anglicisme \\
640 & Barbarisme, pléonasme et tautologie \\
650 & Niveau de langue \\
699 & Inclassable \\
\hline
\end{tabular}

\begin{tabular}{|cl|}
\hline 700 & STYLISTIQUE \\
710 & Précision de la langue \\
720 & Concision de la langue \\
730 & Harmonie de la langue \\
\hline
\end{tabular}

\begin{tabular}{|l|l|l|}
\hline 800 & QUALITÉ TEXTUELLE \\
\hline 810 & RÉGLES D'ORGANISATION \\
811 & Introduction au thème \\
812 & Introduction du thème \\
813 & Présentation du plan & \\
814 & Paragraphe & \\
815 & Développement & \\
816 & Conclusion & \\
820 & RÉGLES DE SOUTIEN \\
821 & Transition & \\
822 & Citation \\
823 & Schéma, figure, tableau & \\
824 & Titre et sous-titre & \\
825 & Référence & \\
830 & RÉGLES DU GENRE \\
\hline 900 & QUALITÉ DE LA PENSÉE \\
910 & Choix et netteté des idées \\
920 & Logique et enchânement des idées \\
930 & Profondeur et originalité \\
\hline
\end{tabular}


ANNEXE II

Grille «A» de base

(Boyer, Roy et Roy, 1991) 
10. Orthographe d'usage

11. Cas général

12. Accent

13. Géminée

14. Trait d'union

Fautes:

20. Morphologie

21. Nom

22. Adjectif

23. Déterminant

24. Pronom

25. Verbe

26. Adverbe

27. Préposition

28.1 Conj. de sub

28.2 Conj. de coord

29. Interjection

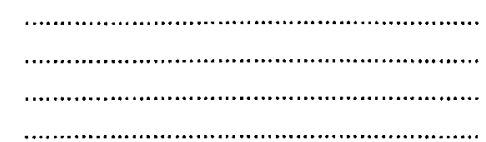

.

Fautes:

30. Accord

31. Nom

36. Adverbe

32. Adjectif

37. Préposition

Déterminant

34. Pronom

39. Interjection

35. Verbe

35.1 Règle générale

35.2 Collectif

38.1 Conj. de sub.

38.2 Conj. de coord.

35.3 Plusieurs sujets

35.4 Part. passé (seul)

35.4 Part. passé (être)

35.6 Part. passé (v. état)

35.5 Part. passé (avoir) 35.9 Autres

Fautes:

40. Structure de la phrase

41. Concordance des temps

42. Référent

43. Mot manquant

44. Mot superflu

45. Mot mal choisi

46. Autres

Fautes:

\section{Ponctuation et majuscule}

51. Point final

52. Virgule

521. De reprise

522. D'explication

523. D'inversion

524. D'ellipse et de retrait

53. Point-virgule

54. Deux-points

55. Points d'interr. ou d'excl

56. Guillemets

57. Trait d'union

58. Majuscule

59. Autres

Fautes:

60 . Lexique

61. Impropriété

62. Anglicisme

63. Barbarisme

Fautes:

Remarques:

Nombre total de fautes:

Correcteur 1:

Correcteur 2: 
ANNEXE III

Grille «B» finale 


\section{GRILLE \\ $\mathrm{DE}$ \\ CORRECTION}

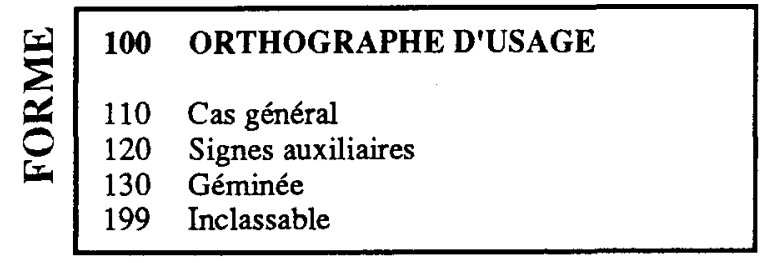

200 MORPHOLOGIE

210 Nom

220 Adjectif qualificatif

230 Article et adj. déterminatif

240 Pronom

250 Verbe

260 Adverbe

270 Préposition

280 Conjonction de subordination

290 Conjonction de coordination

295 Interjection

299 Inclassable

\begin{tabular}{|ll}
\hline 300 & ACCORD \\
310 & Nom \\
320 & Adjectif qualificatif \\
330 & Article et adj. déterminatif \\
340 & Pronom \\
& \\
350 & VERBE: \\
351 & Règle générale \\
352 & Collectif sujet \\
353 & Plusieurs sujets \\
354 & Part. passé (seul) \\
355 & Part. passé (être) \\
356 & Part. passé (avoir) \\
357 & Part. passé (v. pron.) \\
359 & Autre \\
& \\
360 & Adverbe \\
399 & Inclassable
\end{tabular}

400 STRUCTURE DE LA PHRASE

410 Choix du mode et concordance des temps

420 Référent

430 Mot manquant

440 Mot superflu

450 Mot(s) mal choisi(s)

460 Construction fautive

499 Inclassable

\section{SIGNES DE PONCTUATION ET} CONVENTIONS

510 POINT

511 Point final

512 D'interrogation

513 D'exclamation

514 Point-virgule

515 Deux-points

519 Point superflu

520 VIRGULE:

521 De reprise

522 D'explication

523 D'inversion

524 D'ellipse et de retrait

529 Virgule superflue

530 Guillemets et parenthèses

540 Majuscule, minuscule et capitale

550 Trait d'union

560 Apostrophe d'élision

599 Inclassable

600 LEXIQUE (vocabulaire)

610 Impropriété

620 Accord conceptuel

630 Anglicisme

640 Barbarisme, pléonasme et tautologie

650 Niveau de langue

699 Inclassable

\section{STYLISTIQUE}

710 Précision de la langue

720 Concision de la langue

730 Harmonie de la langue

\begin{tabular}{|l|l|l|l|l|}
\hline 800 & QUALITÉ TEXTUELLE \\
810 & RËGLES D'ORGANISATION \\
811 & Introduction au thème \\
812 & Introduction du thème \\
813 & Présentation du plan \\
814 & Paragraphe \\
815 & Développement \\
816 & Conclusion \\
820 & RÉGLES DE SOUTIEN \\
821 & Transition \\
822 & Citation \\
823 & Schéma, figure, tableau \\
824 & Titre et sous-titre \\
825 & Référence \\
830 & RÉGLES DU GENRE \\
\hline 900 & QUALITÉ DE LA PENSÉE \\
910 & Choix et netteté des idées \\
920 & Logique et enchaînement des idées \\
930 & Profondeur et originalité \\
\hline
\end{tabular}




\section{ANNEXE IV}

\section{Exemples d'exercices correctifs}

pour les catégories 400, 500 et 700 


\section{EXERCICES - STRUCTURE DE LA PHRASE \\ CATÉGORIE 400}

\section{0 - RÉFÉRENT}

Les fautes de référent sont les fautes occasionnées par un choix fautif de mot eu égard au rapport que le mot entretient avec d'autres mots de la phrase ou d'une phrase précédente. La faute peut également originer du fait que le ou les mots auxquels il est fait référence ne sont pas présents dans le texte. Il peut être possible, par exemple, que le pronom utilisé ne réfère a aucun mot dans la phrase. La faute de référent peut donc tenir au fait que le référent est ou soit fautif, ou soit absent.

A - Trouvez, dans ces phrases, les fautes de référent et tentez de réécrire les phrases pour en corriger les erreurs.

1 - Pierre a volé Paul; il a porté plainte.

2 - Le garçon a aperçu un ami. Il a traversé la rue.

3- Il se livre à l'ivrognerie. Cela le ruinera.

4 - Jean ă donné à Paul son cadeau.

page 1

Travaux de recherche réalisés par Nathalie Simard sous la direction de Raymond Claude Roy. Maîtrise en Éducation, Université du Québec à Chicoutimi, version septembre 1993. 


\section{EXERCICES - STRUCTURE DE LA PHRASE \\ CATÉGORIE 400}

\begin{tabular}{|ll|}
\hline $450-\quad$ MOT(S) & MAL CHOISI(S) \\
\hline
\end{tabular}

La sous-catégorie du ou des mots mal choisis permet d'identifier les fautes reliées au problème de choix d'un ou de plusieurs mots ou locution qui sont inadéquats de par leur fonction.

A - Trouvez le ou les mots mal choisis et réécrire la phrase en utilisant le ou les mots corrects.

1 - Le tonnerre est tombé.

2- Faire don d'un porte-cigares.

3 - Rompre une croûte.

4 - Ne gardez pas le bien d'autrui.

5 - Ils sont les deux ensembles.

6 - Cette personne est à l'ordre.

7 - Son cheminement de sa vie est très sain.

page 2

Travaux de recherche réalisés par Nathalie Simard sous la direction de Raymond Claude Roy. Maîtrise en Éducation, Université du Québec à Chicoutimi, version septembre 1993. 


\section{0- LES VIRGULES}

Grevisse (1980) et Goosse (1986) dans le Bon usage présentent différents cas d'emploi de la virgule. Toutefois le relevé qui en est fait, même s'il est détaillé, ne constitue pas un guide satisfaisant pour le scripteur cherchant à utiliser adéquatement ce signe de ponctuation.

Par ailleurs, il a souvent été enseigné de placer une virgule lorsque le besoin de faire une pause se fait sentir. Une règle qui ne manque pas d'être insuffisante et qui fait courir beaucoup de risques d'erreurs. Pour maîtriser l'usage du petit signe fort complexe qu'est la virgule, il importe, d'une part, de maîtriser et de distinguer les emplois à faire de la virgule et, d'autre part, d'en comprendre, malgré les difficultés, la logique.

Pour ces raisons, il est proposé quatre types nets d'emploi de la virgule: virgule de reprise, virgule d'explication, virgule d'inversion et virgule d'ellipse et de retrait. Ces derniers seront expliqués, puis des exercices seront proposés pour en permettre une maîtrise. 


\section{1 - VIRGULE DE REPRISE}

La virgule de reprise s'emploie généralement pour marquer les reprises d'éléments semblables. Ces éléments peuvent être des noms, des verbes, des adjectifs, des sujets, des propositions, etc.

\section{La virgule de reprise s'utilise:}

A) Pour séparer les mots ou groupes de mots dont la reprise n'est pas portée par une conjonction de coordination.

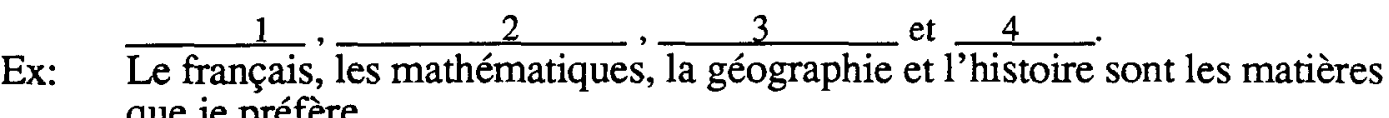
que je préfère.

B) Pour séparer plusieurs propositions coordonnées de même nature.

Ex: $\frac{\text { proposition }}{\text { Je travaille trop, }}, \frac{\text { proposition }}{\text { jeai pas le temps pour mes loisirs. }}$

\section{EXERCICES}

Ponctue les phrases qui suivent en inscrivant la ou les virgules de reprise appropriées et indique, entre les parenthèses, à quel cas d'emploi (A ou B) les virgules utilisées réfèrent.

1- J'ai visité les palais les boutiques les églises etc. ( )

2- Plus on est nombreux plus on s'amuse. ( ) 


\section{2 - VIRGULE D'EXPLICATION}

La virgule d'explication est généralement employée pour détacher tout élément ayant une valeur explicative.

\section{La virgule d'explication s'utilise:}

A) Pour séparer tout élément ayant une valeur explicative, ce qui est fait le plus souvent à l'aide de deux virgules.

Ex: $\quad \stackrel{\text { A treize ans, élément explicatif }}{\text { bientôt quatorze, elle paraissait en avoir dix-huit. }}$

B ) Pour détacher une proposition explicative.

, proposition explicative,

Ex: $\overline{\text { Bernard, }}$, qui attendait son ami, fut surpris par la tempête.

C) Pour marquer la valeur explicative des propositions introduites par une conjonction de coordination autre que "et", "ou", "ni".

, car

Ex: Je ne peux oser faire ce geste, car les conséquences pourraient en être néfastes.

\section{EXERCICES}

Ponctue les phrases ci-bas en inscrivant la ou les virgules d'explication appropriées et indique, entre les parenthèses, à quel cas d'emploi $(\mathrm{A}, \mathrm{B}$ ou $\mathrm{C})$ les virgules utilisées réfèrent.

1 - Annie la cousine était venue rendre visite à Lisa pour son anniversaire. ( )

2- Il y a une raison mais je ne peux la trouver. ( )

3- Ma grand-mère issue d'une famille nombreuse et pauvre n'a pu faire de longues études. ( )

Travaux de recherche réalisés par Nathalie Simard sous la direction de Raymond Claude Roy. Maîtrise en Éducation, Université du Québec à Chicoutimi, version du 30 septembre 1994. 


\section{4- VIRGULE D'ELLIPSE ET DE RETRAIT}

La virgule d'ellipse et de retrait est utilisée pour placer certains mots ou groupes de mots en retrait. Elle sert à isoler un ou des mots mis en apostrophe ou à marquer l'élision d'un mot.

\section{Les virgules d'ellipse et de retrait s'utilisent:}

A) Pour isoler les mots mis en apostrophe.

Ex: $\quad \frac{\text { Mot }}{\text { Chien, rentre dans ta niche! }}$

B) Pour séparer la proposition incise.

Ex: $\quad \overline{\text { Il me plaît, voyez-vous, }}, \overline{\text { d'assister à ce spectacle. }}$

C) Pour marquer l'ellipse d'un verbe ou d'autres mots.

(élision du verbe)

Ex: $\quad \overrightarrow{\text { Le vent était doux et le soleil, brûlant. }}$

\section{EXERCICES}

Ponctue les phrases suivantes à l'aide d'une virgule d'ellipse ou de retrait et indique, entre les parenthèses, à quel cas d'emploi (A, B ou C) la ou les virgules utilisées réfèrent.

1 - Grammaire tu nous étourdis! ( )

2 - Un instant dit-il je suis occupé. ( )

3- Paul s'amuse au parc et Martha à la piscine. ( ) 


\section{EXERCICES:}

Ponctue à l'aide des virgule les vingt-cing phrases suivantes et indique, entre les parenthèses, le type de virgule emplové (521, 522,523 ou 524). Appose un " $X$ " s'il ne convient pas d'ajouter de virgules.

1- Avant qu'il n'arrive je pleurais. ( )

2- Il avait passé des heures peut-être la nuit entière à tenter d'élucider ce mystère. ( )

3- Marco connaissait les alentours par coeur le chemin longeant l'aboiement des chiens la couleur des maisons les arbres et les gens. ( )

4- En effet je désire occuper ce poste. ( )

5 - Rita née en 1984 célèbre cette année son centième anniversaire de naissance. ( )

6- A peine le jour levé nous étions réveillés. ( )

7. Ni toi ni moi n'avons accepté l'invitation. ( )

8- La mère travaille depuis très longtemps alors que la fille qui porte toujours de belles toilettes est encore sans emploi. ( ) ( )

9- Un soir d'avril il entendit des pas à l'intérieur de la maison. ( )

10- Par ailleurs le domaine de l'enseignement est menacé. ( ) 
11. Dans dix jours je partirai. ( )

12 - J'accompagnerai ou mon père ou mon frère à ce mariage. ( )

13. Je ne pourrai pas me libérer alors allez-y sans moi. ( )

14- Il s'arrêta et repartit. ( )

15- Aussitôt arrivées elles se mirent au travail. ( )

16- Chaque parti politique veut défendre notre nation et nos droits. ( )

17- Le champagne le caviar la musique et les amis seront de la fête. ( )

18. Personne ne pourra me faire changer d'idée. ( )

19- Trois minutes monsieur! ( )

20 - Dans la forêt c'était la chasse à l'orignal. ( )

21- Il se fait tard je dois rentrer. ( )

22- Les jeunes aiment les sucreries. ( )

23- Des enfants et des adultes ont été brûlées par les BPC des maisons abandonnées et des souvenirs déchus. $(\quad)(\quad)($ )

24- Chicoutimi le 9 septembre 1994 16h00. ( )

25- Après le départ d'Émilie plusieurs gamins se mirent à rire. ( ) 


\section{EXERCICES DE STYLISTIOUE}

\section{CATÉGORIE 700}

\section{\begin{tabular}{|lll}
\hline $710-$ & Précision du style
\end{tabular}}

A - Précision des termes: AVOIR-Substituer l'un des verbes suivants au verbe "avoir" ou à la locution contenant "avoir".

$$
\begin{array}{ll}
\text { Compter } & \text { Jouir } \\
\text { Occuper } & \text { Remporter }
\end{array}
$$

A 1 - Cet homme (a) une bonne réputation.

A2 - Ce professeur (a) vingt ans de service.

A3- Cet étudiant a (eu) un premier prix.

A4- Il (a) un poste d'importance.

B - $\quad$ ETTRE- Remplacer le verbe ou la locution par l'un des verbes suivants:

Monter Précéder

Concorder Figurer

B1 - Leurs opinions (sont les mêmes).

B2- Son nom n'(est) pas sur la liste des candidats.

B3 - Ce concurrent (est sur) un cheval fougueux.

B4- Le complément (est avant) le verbe. 
C- FAIRE- Remplacer le verbe "faire" par l'un des verbes suivants:

$\begin{array}{ll}\text { Tirer } & \text { Intenter } \\ \text { Prêter } & \text { Pratiquer }\end{array}$

C1 - Faire une ligne.

C2- Faire un procès à quelqu'un.

C3- Faire serment.

C4- Faire un métier passionnant.

D - CHOSE- Remplacer les termes vagues par l'un des noms suivants:

$\begin{array}{ll}\text { Pensée } & \text { Don } \\ \text { Nouvelle } & \text { Obstacle }\end{array}$

D1 - Une bonne mémoire est une chose précieuse.

D2- Une seule chose occupe son esprit.

D3- J'ai appris une chose bien triste.

D4- Une chose l'arrête. 
E- Connaissance du terme propre (étendue du vocabulaire). Préciser la nature du mot à l'aide des choix suivants:
Abstrus
Oiseux
Inique
Fallacieux

E1- Un raisonnement obscur difficile à comprendre.

(Un raisonnement__

E2- Un exposé obscur difficile à comprendre.

(Un exposé _ )

E3- Une loi qui n'observe pas la justice naturelle.

(une loi

E4- Un discours inutile, qui n'aboutit à rien.

(Un discours 


\section{$720-\quad$ Concision}

F - Souligner le mot lexiquement ou logiquement superflu.

F1 - Au grand maximum...

F2- Une très infime partie...

F3- Il y a environ trente ou quarante ans.

F4- Être superposés les uns sur les autres.

F5- Ainsi par exemple...

F6- La fin du récit se termine sur une réconciliation.

F7- Il suffit simplement de la faire.

G- Souligner les mots grammaticalement superflus qui, cette foisci, relèveront de la sous-catégorie 440 (mot superflu en structure de phrase).

G1- Quiconque qui 1'a entendu...

G2- Vous ne commanderez seulement que les articles intéressants.

H - Réécrire les expressions sans les pléonasmes:

H1 - Au jour d'aujourd'hui.

H2 - Une hémorragie de sang.

H3 - Prévoir à l'avance.

H4- Monter en haut. 
I - Économie de termes: Réécrire la phrase de façon à faire une économie de termes.

I 1 - Nombre de personnes se figurent être le centre du monde, autour duquel tout gravite (Mottoule, 1957).

I2- Chacun de nous, ici-bas, traîne après soi son bagage personnel de défauts (Idem).

I3 - Il esquisse une moue de visage (Idem).

I4- La période trouble que nous traversons actuellement a bouleversé bien des projets (Idem).

J - Épuration rigoureuse (redondance). Tenter de réécrire cette phrase de façon à éliminer la redondance.

J1 - Plus la civilisation humaine cherche à enfermer davantage les hommes dans son système de contrôle et d'organisation méthodique, plus les hommes cherchent à échapper le plus qu'il se peut à son étreinte, comme s'ils considéraient qu'il y va de leur existence morale, pour ne pas dire de leur droit à la vie ici-bas.

Travaux de recherche réalisés par Nathalie Simard sous la direction de Raymond Claude Roy. Maîtrise en Éducation, Université du Québec à Chicoutimi, version septembre 1993. 


\section{0- Harmonie}

K- Les phrases suivantes contiennent des éléments cacophoniques. Refaites les phrases de façon à les rendre harmonieuses.

(Cacophonie: Rencontre ou répétition de sons désagréables ou ridicules dans le discours.)

K1 - Il préfère faire ses affaires seul.

K2- En en entendant parler...

K3- A l'exception de la dernière, l'Académie accepta toutes les acceptions nouvelles du terme.

L - Disposition et ordre des mots. Réécrire les phrases dans un ordre où les mots seront logiquement disposés. L'erreur relève proprement de la sous-catégorie 460 (construction fautive de la phrase).

L4-. Je garde le souvenir d'années tranquilles de mon enfance.

L5- Mon père s'était inquiété de ma vie de dissipation dans sa demi-retraite.

L6 - J'ouvris la fenêtre de ma chambre sur la nuit. 


\section{ANNEXE V}

\section{Portrait diagnostique}

de groupe 


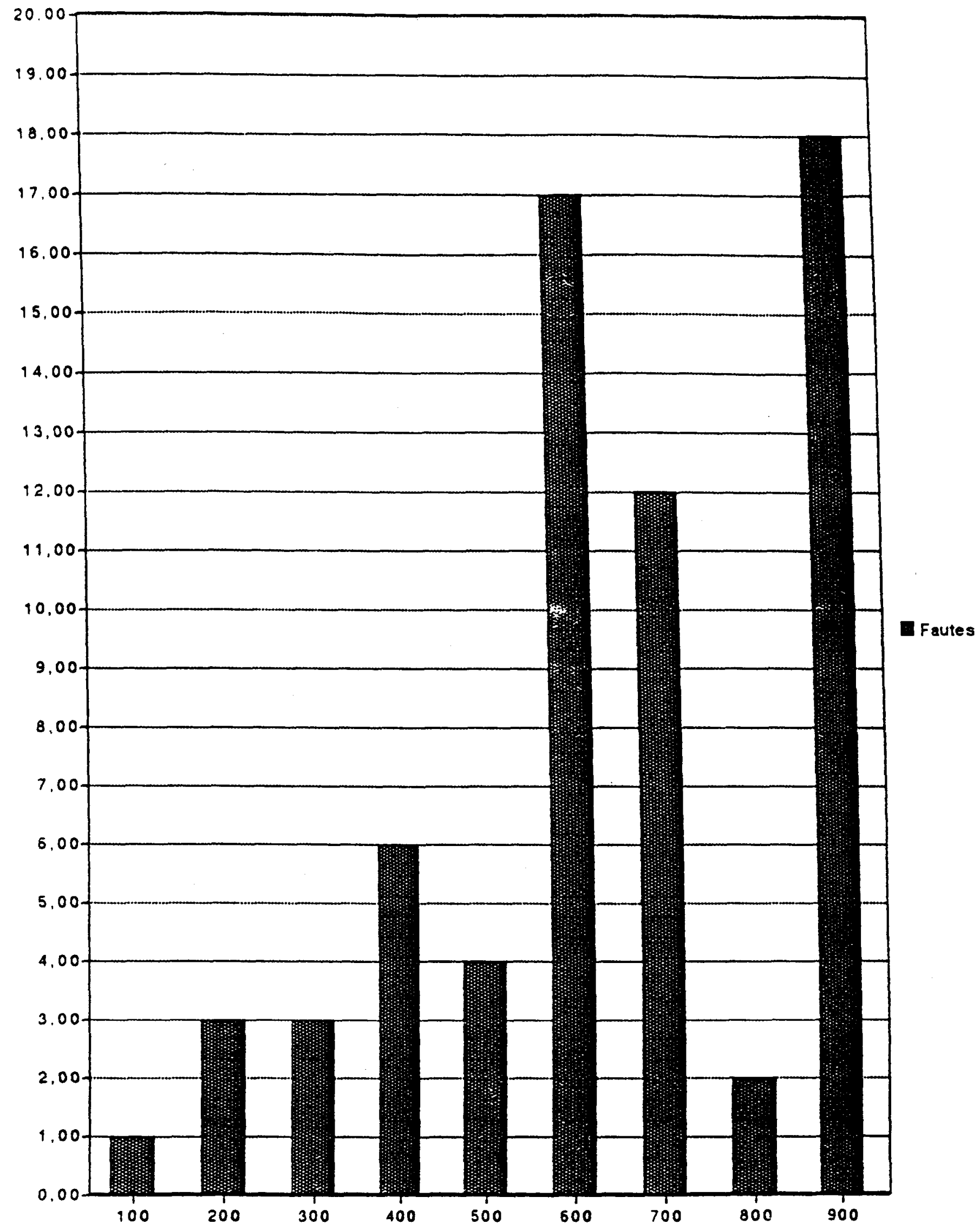




\section{ANNEXE VI}

Portrait diagnostique individuel 


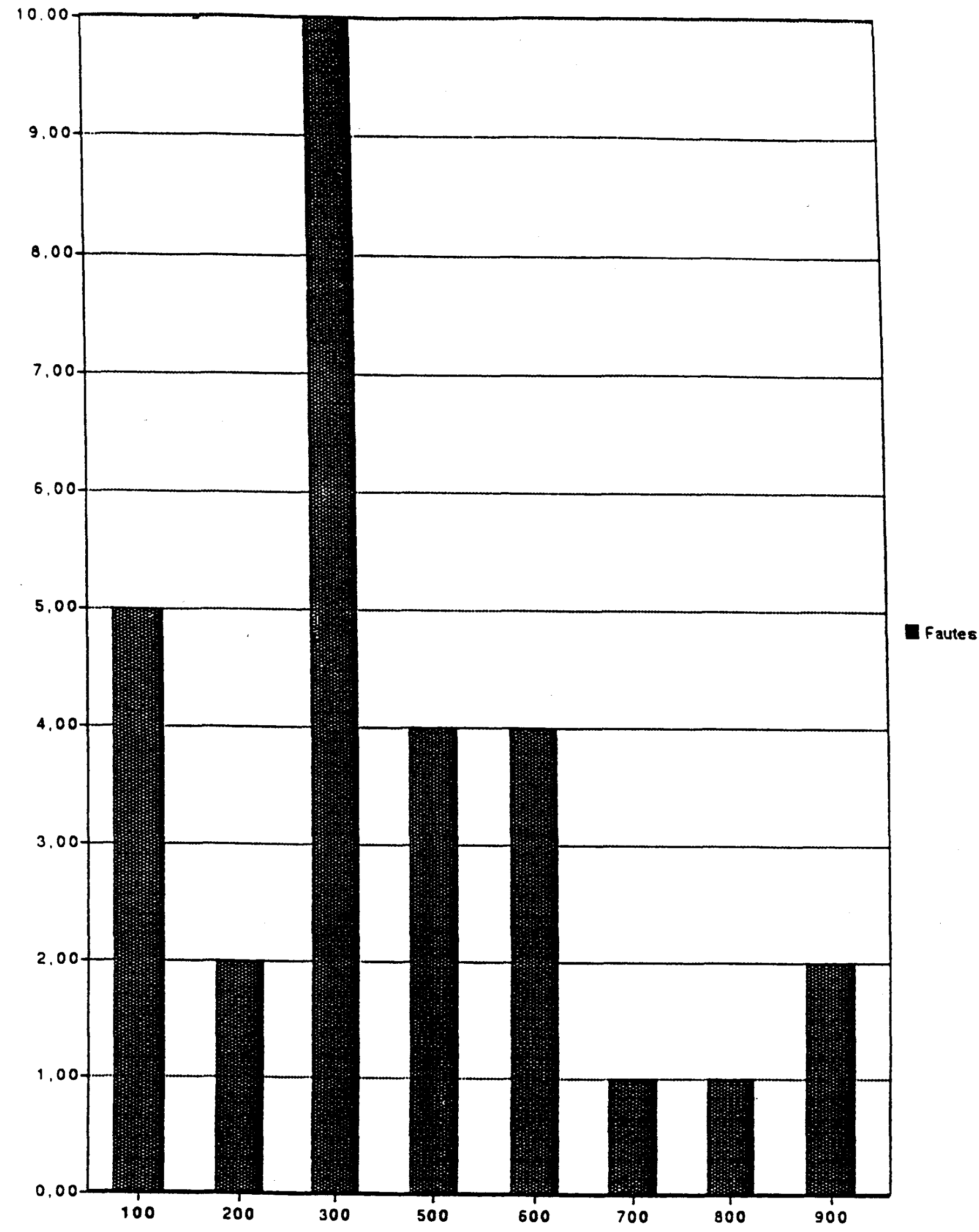

